\title{
Cauchy Problem for Semilinear Parabolic Equation with Time-Dependent Obstacles: A BSDEs Approach
}

\author{
Tomasz Klimsiak
}

Received: 23 December 2011 / Accepted: 30 October 2012 / Published online: 22 November 2012

(C) The Author(s) 2012. This article is published with open access at Springerlink.com

\begin{abstract}
In the paper we apply methods of the theory of backward stochastic differential equations to prove existence, uniqueness and stochastic representation of solutions of the Cauchy problem for semilinear parabolic equation in divergence form with two time-dependent obstacles. We consider two quite different cases: problems with distinct quasi-continuous obstacles and with irregular obstacles satisfying the so called Mokobodzki condition. As an application we also generalize the Lewy-Stampacchia inequality to non-Radon measures and give new existence result for the Dynkin game problem.
\end{abstract}

Keywords Semilinear parabolic equation - Divergence form operator • Obstacle problem $\cdot$ Backward stochastic differential equation

Mathematics Subject Classifications (2010) Primary 35K86; Secondary 60H99

\section{Introduction}

Let $Q_{T}=[0, T] \times \mathbb{R}^{d}$. Suppose we are given a final condition $\varphi: \mathbb{R}^{d} \rightarrow \mathbb{R}$, coefficient $f: Q_{T} \times \mathbb{R} \times \mathbb{R}^{d} \rightarrow \mathbb{R}$ and two functions $h_{1}, h_{2}: Q_{T} \rightarrow \mathbb{R}$ called obstacles or barriers. The problem under consideration may be formulated as the semilinear parabolic absorption problem of the form

$$
\begin{cases}u_{t}+L_{t} u-\beta(t, x, u) \ni-f(t, x, u, \nabla u) & \text { in }[0, T) \times \mathbb{R}^{d} \\ u(T, \cdot)=\varphi & \text { on } \mathbb{R}^{d}\end{cases}
$$

Research supported by the Polish Minister of Science and Higher Education under Grant N N201 372436.

T. Klimsiak $(\varangle)$

Faculty of Mathematics and Computer Science, Nicolaus Copernicus University,

Chopina 12/18, 87-100 Toruń, Poland

e-mail: tomas@mat.uni.torun.pl 
where $\beta$ is the subdifferential of $j$ defined by $j(t, x, \cdot)=\mathbf{I}_{\left[h_{1}(t, x), h_{2}(t, x)\right]}(\cdot)$. In the whole paper we assume that the operator $L_{t}$ is of the form

$$
L_{t}=\frac{1}{2} \sum_{i, j=1}^{d} \frac{\partial}{\partial x_{i}}\left(a_{i j}(t, x) \frac{\partial}{\partial x_{j}}\right)+\sum_{i=1}^{d} b_{i}(t, x) \frac{\partial}{\partial x_{i}},
$$

and its coefficients $a: Q_{T} \rightarrow \mathbb{R}^{d} \otimes \mathbb{R}^{d}, b: Q_{T} \rightarrow \mathbb{R}^{d}$ are measurable functions such that

$$
\lambda|\xi|^{2} \leq \sum_{i, j=1}^{d} a_{i j}(t, x) \xi_{i} \xi_{j} \leq \Lambda|\xi|^{2}, \quad a_{i j}=a_{j i}, \quad\left|b_{i}\right| \leq \Lambda, \quad \xi \in \mathbb{R}^{d}
$$

for some $0<\lambda \leq \Lambda$. We assume that the data $\varphi, f$ satisfy the following hypotheses:

(H1) $\varphi \in \mathbb{L}_{2, \varrho}\left(\mathbb{R}^{d}\right)$,

(H2) $f:[0, T] \times \mathbb{R}^{d} \times \mathbb{R} \times \mathbb{R}^{d} \rightarrow \mathbb{R}$ is a measurable function such that there exist $M>0$ and $g \in \mathbb{L}_{2, \varrho}\left(Q_{T}\right)$ such that $|f(t, x, y, z)| \leq g(t, x)+M(|y|+|z|)$ for all $(t, x, y, z) \in[0, T] \times \mathbb{R}^{d} \times \mathbb{R} \times \mathbb{R}^{d}$,

(H3) there is $L>0$ such that $\left|f\left(t, x, y_{1}, z_{1}\right)-f\left(t, x, y_{2}, z_{2}\right)\right| \leq L\left(\left|y_{1}-y_{2}\right|+\mid z_{1}-\right.$ $\left.z_{2} \mid\right)$ for all $(t, x) \in Q_{T}, y_{1}, y_{2} \in \mathbb{R}$ and $z_{1}, z_{2} \in \mathbb{R}^{d}$.

As for the obstacles, we consider two essentially different cases: obstacles satisfying the condition

(H4) $h_{1}, h_{2} \in \mathcal{S}_{\varrho}^{2}, h_{1}<h_{2}$ q.e. and $h_{1}(T, \cdot) \leq \varphi \leq h_{2}(T, \cdot)$ a.e.,

or the condition

(H4*) $h_{1}, h_{2}$ are measurable functions and there exist $\phi^{+}, \phi^{-} \in \mathcal{P}$ such that $h_{1} \leq \phi \leq$ $h_{2}$ a.e., where $\phi=\phi^{+}-\phi^{-}$.

Here $\mathcal{S}_{\varrho}^{2}$ is the space of quasi-continuous functions on $Q_{T}$ satisfying some mild integrability condition (it is satisfied for instance by obstacles which grow at most polynomially), $\varrho$ is some weight and $\mathcal{P}$ is the set of parabolic potentials (definitions of $\mathcal{P}, \mathcal{S}_{\varrho}^{2}$ are given in Sections 2 and 3).

In spite of enormous literature on obstacle problems, parabolic semilinear problem with two time-dependent obstacles is insufficiently investigated. In general, if coefficients of the operator and obstacles depend on time, existence and uniqueness of a solution is known only in the case of regular barriers (see the classical monograph [5] or more recent papers [9, 18, 19, 27]). One of the main reason for lack of satisfactory results for time-dependent irregular barriers are difficulties in proper formulation of the problem to get uniqueness.

An interesting approach to problems of the form (1.1) is proposed in [1]. From [1] it follows in particular that if $D \subset \mathbb{R}^{d}$ is a bounded domain, $b=0$ and $a$ does not depend on time then for $f \in \mathbb{L}_{1}(\mathcal{Q})$, where $\mathcal{Q}=(0, T) \times D$, there exists a unique solution of the Cauchy-Dirichlet problem

$$
\begin{cases}u_{t}+L_{t} u-\beta(x, u) \ni-f(t, x) & \text { in } \mathcal{Q}, \\ u=0 & \text { on }(0, T) \times \partial D \\ u(T, \cdot)=\varphi & \text { on } D\end{cases}
$$


with irregular but time-independent obstacles such that $h_{1} \leq 0 \leq h_{2}$ a.e.. In [1] to cope with Eq. 1.4 variational equalities with some soft measure on the right-hand side rather then variational inequalities are studied. A solution of Eq. 1.4 is defined as a pair $(u, \mu)$, where $u \in C\left([0, T] ; \mathbb{L}_{2}(D)\right) \cap \mathbb{L}_{2}\left(0, T ; H_{0}^{1}(D)\right), \mu \in \mathbb{L}_{1}\left(0, T ; \mathcal{M}_{0}(D)\right)$ $\left(\mathcal{M}_{0}(D)\right.$ is the set of bounded smooth measures on $D$ equipped with the topology of weak* convergence) such that for every $\eta \in \mathcal{W}(\mathcal{Q})$ (i.e. $\eta \in \mathbb{L}_{2}\left(0, T ; H_{0}^{1}(D)\right.$ ) such that $\left.\frac{\partial \eta}{\partial t} \in \mathbb{L}_{2}\left(0, T ; H^{-1}(D)\right)\right)$ with $\eta(0) \equiv 0$,

$$
\left\langle u, \frac{\partial \eta}{\partial t}\right\rangle_{T}-\left\langle L_{t} u, \eta\right\rangle_{T}=(\varphi, \eta(T))+(f, \eta)_{T}+\int_{0}^{T} \int_{D} \tilde{\eta}(t) d \mu(t) d t
$$

and

$$
\begin{cases}\mu_{r}(t) \in \partial j(\cdot, u(t))+\partial \mathbf{I}_{\left[\gamma_{-}(\cdot), \gamma_{+}(\cdot)\right]} & \text { for a.e. } t \in[0, T], \\ \tilde{u}(t)=\gamma_{+}, \mu_{s}^{-}(t) \text {-a.e., } \quad \tilde{u}(t)=\gamma_{-}, \mu_{s}^{+} \text {-a.e. } & \text { for a.e. } t \in[0, T],\end{cases}
$$

where $\tilde{u}(t)$ is a quasi-continuous version of $u(t), \mu(t)=\mu_{r}(t)+\mu_{s}(t)$ is the RadonNikodym decomposition of the measure $\mu(t)$ and $\gamma_{+}, \gamma_{-}$are unique, in the quasieverywhere sense, quasi-l.s.c and quasi-u.s.c. functions, respectively, determined by $j$ (see [1] for details). One can check that in the case $j(x, \cdot)=\mathbf{I}_{\left[h_{1}(x), h_{2}(x)\right]}(\cdot)$, condition (1.6) is equivalent to the so-called minimality condition

$$
\int_{0}^{T} \int_{D}\left(\tilde{u}(t)-\gamma_{-}\right) d \mu^{+}(t) d t=\int_{0}^{T} \int_{D}\left(\tilde{u}(t)-\gamma_{+}\right) d \mu^{-}(t) d t=0 .
$$

(Here $\mu^{-}, \mu^{+}$are components of the Jordan decomposition of $\mu$ ).

In the case of one time-dependent barrier similar approach to obstacle problem is presented in the linear case in [31] and in the semilinear case in [17]. As in [1], in $[17,31]$ a solution is a pair $(u, \mu)$ consisting of a function $u$ on $Q_{T}$ having some regularity properties and a smooth measure $\mu$ on $Q_{T}$ such that equation similar to Eq. 1.5 and minimality condition similar to Eq. 1.7 are satisfied. Such a formulation of a solution of the obstacle problem is very useful because instead of variational inequalities we consider variational equalities which provide an additional information on the solution, and what is more important, it guarantees uniqueness of solutions (in the theory of variational inequalities solutions of obstacle problems with timedependent barriers are in general not unique, see e.g. [24]).

In the case of time-dependent barriers considered in the paper the problem of proper formulation of the minimality condition for $\mu$ is more difficult than in the time-independent case considered in [1] because if $h_{1}, h_{2}$ satisfy (H4*) only, then in general neither $u$ belongs to the space $C\left([0, T] ; \mathbb{L}_{2, \varrho}\left(\mathbb{R}^{d}\right)\right)$ nor $\mu$ belongs to $\mathbb{L}_{1}\left(0, T ; \mathcal{M}_{0}\left(\mathbb{R}^{d}\right)\right.$ ) (see Section 5 ). To define properly the minimality condition for $\mu$ we use stochastic approach introduced in [17]. Let $\mathbb{X}=\left\{\left(X, P_{s, x}\right) ;(s, x) \in Q_{\hat{T}}\right\}$ be a Markov family with generator $L_{t}$. In our definition we require that for every $h_{1}^{*}, h_{2}^{*} \in \mathcal{D}_{\varrho}^{2}$ such that $h_{1} \leq h_{1}^{*} \leq \bar{u} \leq h_{2}^{*} \leq h_{2}$ a.e.,

$$
\begin{aligned}
\int_{s}^{T}\left(\bar{u}_{-}\left(t, X_{t}\right)-h_{1-}^{*}\left(t, X_{t}\right)\right) d \mu^{+}\left(t, X_{t}\right) \\
\quad=\int_{s}^{T}\left(\bar{u}_{-}\left(t, X_{t}\right)-h_{2-}^{*}\left(t, X_{t}\right)\right) d \mu^{-}\left(t, X_{t}\right)=0, \quad P_{s, x^{-}} \text {a.s. }
\end{aligned}
$$


for q.e. $(s, x) \in Q_{\hat{T}} \equiv[0, T) \times \mathbb{R}^{d}$. Here $d \mu^{-}\left(\cdot, X\right.$.), $d \mu^{+}(\cdot, X$.) are some random measures which are in the Revuz correspondence (see Eq. 3.2) with $\mu^{-}$and $\mu^{+}$, respectively, $\bar{u}$ is a quasi-càdlàg version of $u$ (i.e. $t \rightarrow \bar{u}\left(t, X_{t}\right)$ is càdlàg $P_{s, x}$-a.s. for q.e. $\left.(s, x) \in Q_{\hat{T}}\right), v_{-}\left(t, X_{t}\right)=\lim _{s \rightarrow t^{-}} v\left(s, X_{s}\right)$ and $\mathcal{D}_{\varrho}^{2}$ is the set of quasi-càdlàg functions on $Q_{T}$ having some integrability properties. Let us remark that the fact that in Eq. 1.8 we take left limits of $\bar{u}$ along trajectories of $X$ is closely related to the concept of precise versions of functions considered in [31] to cope with the linear obstacle problem with time-dependent barrier (see [17, Proposition 5.17]).

In the paper we show that under $(\mathrm{H} 1)-(\mathrm{H} 3),(\mathrm{H} 4 *)$ there exists a unique solution of Eq. 1.1, i.e. there is $(u, \mu)$ such that $h_{1} \leq u \leq h_{2}$ a.e., Eq. 1.5 with $\int_{0}^{T} \int_{D} \tilde{\eta}(t) \varrho^{2} d \mu(t) d t$ replaced by $\int_{Q_{T}} \eta \varrho^{2} d \mu$ and Eq. 1.8 are satisfied (see definition in Section 5.4).

Assumption ( $\mathrm{H} 4)$ is rather uncomparable with $(\mathrm{H} 4 *)$. To our knowledge, it is considered here for the first time in the context of problems of the form (1.1). Roughly speaking, in the literature two types of assumptions on obstacles are considered: regularity of $h_{1}, h_{2}$ in some Sobolev spaces (see [18]) or the separation condition saying that there is some regular function between $h_{1}$ and $h_{2}$ (for instance in [1] the condition $h_{1} \leq 0 \leq h_{2}$ a.e. is assumed). In (H4) we do not impose any regularity assumption on obstacles beside their quasi-continuity. Assumption (H4) does not imply the separation condition. In fact, it may happen that there is no regular function (i.e. function from some Sobolev space) between the obstacles (see Example 5.6).

The last observation is crucial because it forces that in general $u$ is not a Sobolev space solution and $\mu$ is a non-Radon measure (see Example 5.6). To cope with the first problem we have to give meaning to the gradient of $u$. For this purpose we introduce some generalized Sobolev space $W^{0,1}(\mathbb{X})$ defined with the use of the Markov family $\mathbb{X}$. We show that $W^{0,1}(\mathbb{X})$ is a Polish space, $W_{2}^{0,1}\left(Q_{T}\right) \subset W^{0,1}(\mathbb{X})$ and that every $u \in W^{0,1}(\mathbb{X})$ possesses the so-called stochastic gradient $\nabla_{\mathbb{X}} u$. The stochastic gradient has the property that $\nabla_{\mathbb{X}} u=\nabla u$ a.e. if $u \in W_{p}^{0,1}\left(Q_{T}\right) \cap W^{0,1}(\mathbb{X})$ for some $p \geq 1$. Let us remark that in general, $W^{0,1}(\mathbb{X}) \subsetneq \mathcal{T}_{2, \varrho}^{0,1}$ and $\mathcal{T}_{2, \varrho}^{0,1} \subsetneq W^{0,1}(\mathbb{X})$, where $\mathcal{T}_{2, \varrho}^{0,1}$ is the space of functions introduced in [4] to investigate existence and uniquenes of entropy or renormalized solutions of PDEs with $\mathbb{L}_{1}$ or measure data (see Remark 4.7).

Since in general $\mu$ is not a Radon measure, one cannot consider variational equality (1.5) or some its modifications involving truncations of $u$ as in the theory of entropy or renormalized solutions. Under (H4) we define a solution of Eq. 1.1 as a pair $(u, \mu)$ such that $u \in W^{0,1}(\mathbb{X}), \mu$ is a smooth measure, i.e. measure which charges no sets of zero parabolic capacity, the minimality condition (1.8) is satisfied and a generalized backward stochastic differential equation (BSDE) associated with Eq. 1.5, namely equation of the form

$$
\begin{aligned}
u\left(t, X_{t}\right)= & \varphi\left(X_{T}\right)+\int_{t}^{T} f_{u}\left(\theta, X_{\theta}\right) d \theta+\int_{t}^{T} d \mu\left(\theta, X_{\theta}\right) \\
& -\int_{t}^{T} \sigma \nabla_{\mathbb{X}} u\left(\theta, X_{\theta}\right) d B_{s, \theta}, \quad t \in[s, T], \quad P_{s, x} \text {-a.s. }
\end{aligned}
$$

is satisfied for q.e. $(s, x) \in Q_{\hat{T}}$. Here $f_{u}(t, x)=f\left(t, x, u(t, x), \nabla_{\mathbb{X}} u(t, x)\right), a=\sigma \sigma^{T}$ and $B_{s}$, is some standard Wiener process starting from time $s$. We show that under (H1)- 
(H4) problem (1.1) has a unique solution, $u$ is quasi-continuous and the minimality condition (1.8) is equivalent to condition that $P_{s, x}$-a.s.,

$\int_{s}^{T}\left(\bar{u}\left(t, X_{t}\right)-h_{1}\left(t, X_{t}\right)\right) d \mu^{+}\left(t, X_{t}\right)=\int_{s}^{T}\left(\bar{u}\left(t, X_{t}\right)-h_{2}\left(t, X_{t}\right)\right) d \mu^{-}\left(t, X_{t}\right)=0$

for q.e. $(s, x) \in Q_{\hat{T}}$. Notice that Eq. 1.9 together with Eq. 1.10 means that under $P_{s, x}$ the triple

$$
\left(Y^{s, x}, Z^{s, x}, K^{s, x}\right)=\left(u(\cdot, X .), \sigma \nabla_{\mathbb{X}} u(\cdot, X .), \int_{s} d \mu\left(t, X_{t}\right)\right)
$$

is a solution of BSDE with the final condition $\varphi\left(X_{T}\right)$, coefficient $f$ and two reflecting barriers $h_{1}(\cdot, X)$ and $h_{2}(\cdot, X)$.

In case $(\mathrm{H} 1)-(\mathrm{H} 3),\left(\mathrm{H} 4^{*}\right)$ are satisfied, Eq. 1.9 holds with $u, \nabla_{\mathbb{X}} u$ replaced by $\bar{u}$, $\nabla u$, which together with Eq. 1.8 means that also in that case $u$ may be represented by a solution of doubly reflected BSDE with final condition $\varphi\left(X_{T}\right)$, coefficient $f$ and barriers $h_{1}(\cdot, X), h_{2}(\cdot, X)$. It is worth mentioning that if $(\mathrm{H} 1)-(\mathrm{H} 3),(\mathrm{H} 4 *)$ are satisfied then Eq. 1.5 is equivalent to Eq. 1.9. From Eq. 1.9 it follows in particular that

$$
u(s, x)=E_{s, x}\left(\varphi\left(X_{T}\right)+\int_{s}^{T} f_{u}\left(t, X_{t}\right) d t+\int_{s}^{T} d \mu\left(t, X_{t}\right)\right)
$$

for q.e. $(s, x) \in Q_{\hat{T}}$, so Eq. 1.9 may be considered as some nonlinear generalization of the Feynman-Kac formula. Representation similar to Eq. 1.11 holds also in case (H1)-(H4) are satisfied (see Remark 5.2 for details).

As a consequence of existence and uniqueness results for Eq. 1.1 we generalize the Lewy-Stampacchia inequality to non-Radon measures and we obtain new existence result for the Dynkin game problem. The last result says that under $(\mathrm{H} 1)-(\mathrm{H} 4)$ the value function

$$
\begin{aligned}
u(s, x)= & \inf _{\sigma \in \mathcal{T}^{s}} \sup _{\tau \in \mathcal{T}^{s}} E_{s, x}\left(\int_{s}^{\tau \wedge \sigma} f_{u}\left(\theta, X_{\theta}\right)\right) d \theta+h_{1}\left(\tau, X_{\tau}\right) \mathbf{1}_{\{\tau \leq \sigma<T\}} \\
& \left.+h_{2}\left(\sigma, X_{\sigma}\right) \mathbf{1}_{\{\sigma<\tau\}}+\varphi\left(X_{T}\right) \mathbf{1}_{\{\sigma=\tau=T\}}\right),
\end{aligned}
$$

where $\mathcal{T}^{s}$ denotes the set of all $\left\{\mathcal{G}_{t}^{s}\right\}$-stopping times and $\left\{\mathcal{G}_{t}^{s}\right\}$ is a minimum completed admissible filtration for $\left(X, P_{s, x}\right)$, is quasi-continuous and coincides with the first component of a solution of Eq. 1.1.

We have already mentioned that problem (1.1) is closely related to reflected BSDEs. Some general results on such equations based on the papers [29, 30] are given in Section 6. Finally, let us mention that in the case where $L_{t}$ is a nondivergent form operator with continuous coefficients, relation between reflected BSDEs with two time-dependent continuous barriers and viscosity solutions of the associated obstacle problem has been investigated in $[8,15]$.

Notations $Q_{T}=[0, T] \times \mathbb{R}^{d}, \quad Q_{s t}=[s, t] \times \mathbb{R}^{d}, \quad Q_{\hat{T}}=[0, T) \times \mathbb{R}^{d}, \check{Q}_{T}=(0, T) \times$ $\mathbb{R}^{d}$. By $m_{T}, m, \lambda$ we denote the Lebesgue measure on $Q_{T}, \mathbb{R}^{d}, \mathbb{R}$, respectively.

Let $\varrho: \mathbb{R}^{d} \rightarrow \mathbb{R}$ be some weight. $\mathbb{L}_{p, \varrho}\left(\mathbb{R}^{d}\right)\left(\mathbb{L}_{p, \varrho}\left(Q_{T}\right)\right)$ is the space of measurable functions $u$ on $\mathbb{R}^{d}\left(Q_{T}\right)$ such that $u \varrho$ is $p$-integrable. By $\|\cdot\|_{p, \varrho}\left(\right.$ resp. $\left.\|\cdot\|_{p, \varrho, T}\right)$ we denote the usual norm in $\mathbb{L}_{p, \varrho}\left(\mathbb{R}^{d}\right)\left(\operatorname{resp} . \mathbb{L}_{p, \varrho}\left(Q_{T}\right)\right)$ and by $(\cdot, \cdot)_{2, \varrho},\left(\operatorname{resp} .(\cdot, \cdot)_{2, \varrho, T}\right)$ the usual inner product in $\mathbb{L}_{2, \varrho}\left(\mathbb{R}^{d}\right)$, (resp. $\left.\mathbb{L}_{2, \varrho}\left(Q_{T}\right)\right)$. If $\varrho \equiv 1$ we drop it in the 
notation of the space. $\mathbb{L}_{p}^{\text {loc }}\left(Q_{T}\right)$ is the space of measurable functions $u$ on $Q_{T}$ such that $\mathbf{1}_{K} u \in \mathbb{L}_{p}\left(Q_{T}\right)$ for every compact set $K \subset Q_{T}$.

$W_{p, \varrho}^{1}\left(\right.$ resp. $\left.W_{p, \varrho}^{0,1}\right)$ is the Banach space of all $u \in \mathbb{L}_{p, \varrho}\left(\mathbb{R}^{d}\right)\left(\right.$ resp. $\left.u \in \mathbb{L}_{p, \varrho}\left(Q_{T}\right)\right)$ heaving generalized derivatives $\frac{\partial u}{\partial x_{i}}, i=1, \ldots, d$ from $\mathbb{L}_{p, \varrho}\left(\mathbb{R}^{d}\right)\left(\mathbb{L}_{p, \varrho}\left(Q_{T}\right)\right) . W_{p, \varrho}^{-1}$ $\left(W_{p, \varrho}^{0,-1}\right)$ is the dual space to $W_{p, \varrho}^{1}\left(W_{p, \varrho}^{0,1}\right) . W_{p, \varrho}^{1,1}$ is the subspace of $W_{p, \varrho}^{0,1}$ consisting of all elements $u$ having generalized derivative $\frac{\partial u}{\partial t}$ in $\mathbb{L}_{p, \varrho}\left(Q_{T}\right)$ and $\mathcal{W}_{\varrho}$ is a subspace of $W_{p, \varrho}^{0,1}$ consisting of all elements $u$ such that $\frac{\partial u}{\partial t} \in W_{p, \varrho}^{0,-1}$.

$H=\mathbb{L}_{2, \varrho}\left(\mathbb{R}^{d}\right), \mathcal{H}=\mathbb{L}_{2, \varrho}\left(Q_{T}\right), F=W_{2, \varrho}^{1}\left(\mathbb{R}^{d}\right), \mathcal{F}=W_{2, \varrho}^{0,1}\left(Q_{T}\right)$ and $\|\cdot\|_{H}=\|\cdot\|_{2, \varrho}$, $\|\cdot\|_{\mathcal{H}}=\|\cdot\|_{2, \varrho, T}$. By $\langle\cdot, \cdot\rangle\left(\operatorname{resp} .\langle\cdot, \cdot\rangle_{T}\right)$ we denote the duality pairing between $F$ and its dual space $F^{\prime}\left(\operatorname{resp} . \mathcal{F}\right.$ and $\left.\mathcal{F}^{\prime}\right)$, and we write $(\cdot, \cdot)=\langle\cdot, \cdot\rangle_{2, \varrho}$ and $(\cdot, \cdot)_{T}=\langle\cdot, \cdot\rangle_{2, \varrho, T}$.

$C_{c}(E)$ (resp. $\left.C_{c}^{\infty}(E)\right)$ is the space of continuous (resp. smooth) functions on $E$ with compact support. $\mathcal{M}(E), \mathcal{M}^{+}(E), \mathcal{M}_{b}^{+}(E)$ denote set of Radon measures, nonnegative Radon measures, nonnegative bounded measures on $E$, respectively. $\mathcal{B}(E)$ $\left(\mathcal{B}^{+}(E)\right)$ is the set of Borel measurable functions (nonnegative Borel measurable functions) on $E$.

By $C$ (or $c$ ) we denote a general constant which may vary from line to line but depends only on fixed parameters.

\section{Preliminary Results}

\subsection{Diffusions Corresponding to Divergence form Operators}

Let $\Omega=C\left([0, T], \mathbb{R}^{d}\right)$ denote the space of continuous $\mathbb{R}^{d}$-valued functions on $[0, T]$ equipped with the topology of uniform convergence and let $X$ be the canonical process on $\Omega$. It is known that given $L_{t}$ defined by Eq. 1.2 with $a, b$ satisfying Eq. 1.1 one can construct a weak fundamental solution $p(s, x, t, y)$ for $L_{t}$ and then a Markov family $\mathbb{X}=\left\{\left(X, P_{s, x}\right) ;(s, x) \in[0, T) \times \mathbb{R}^{d}\right\}$ for which $p$ is the transition density function, i.e.

$$
P_{s, x}\left(X_{t}=x ; 0 \leq t \leq s\right)=1, \quad P_{s, x}\left(X_{t} \in \Gamma\right)=\int_{\Gamma} p(s, x, t, y) d y, \quad t \in(s, T]
$$

for any $\Gamma$ in the Borel $\sigma$-field $\mathcal{B}$ of $\mathbb{R}^{d}$ (see $[36,44]$ ). In what follows by $E_{s, x}$ we denote the expectation with respect to $P_{s, x}$.

Remark 2.1 It is known (see, e.g., [37]), that if $\left(X, Q_{s, x}\right)$ is associated with the operator

$$
A_{t}=\sum_{i, j=1}^{d} \frac{\partial}{\partial x_{i}}\left(a_{i j}(t, x) \frac{\partial}{\partial x_{j}}\right),
$$

then $\frac{d Q_{s, x}}{d P_{s, x}}=Z_{T}$, where $Z$ is a solution of the $\mathrm{SDE}$

$$
d Z_{t}=b\left(t, X_{t}\right) \sigma^{-1}\left(t, X_{t}\right) Z_{t} d B_{s, t}, \quad Z_{0}=1
$$

under the measure $P_{s, x}$. From this it follows immediately that for every $p \geq 1$,

$$
\sup _{(s, x) \in Q_{\hat{T}}} E_{s, x} Z_{T}^{p}<\infty .
$$


From now on we say that some property is satisfied quasi-everywhere (q.e. for short) if it is satisfied except for some Borel set of capacity cap ${ }_{\mathcal{L}}$ (see Section 2.3) equal to zero.

Set $\mathcal{F}_{t}^{s}=\sigma\left(X_{u}, u \in[s, t]\right), \overline{\mathcal{F}}_{t}^{s}=\sigma\left(X_{u}, u \in[T+s-t, T]\right)$ and define $\mathcal{G}$ as the completion of $\mathcal{F}_{T}^{s}$ with respect to the family $\mathcal{P}=\left\{P_{s, \mu}: \mu\right.$ is a probability measure on $\left.\mathcal{B}\left(\mathbb{R}^{d}\right)\right\}$, where $P_{s, \mu}(\cdot)=\int_{\mathbb{R}^{d}} P_{s, x}(\cdot) \mu(d x)$, and define $\mathcal{G}_{t}^{s}\left(\overline{\mathcal{G}}_{t}^{s}\right)$ as the completion of $\mathcal{F}_{t}^{s}\left(\overline{\mathcal{F}}_{t}^{s}\right)$ in $\mathcal{G}$ with respect to $\mathcal{P}$. We will say that a family $A=\left\{A_{s, t}, 0 \leq s \leq t \leq T\right\}$ of

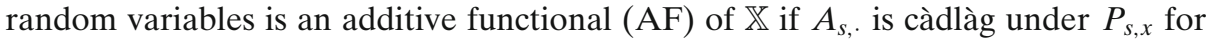
q.e. $(s, x) \in Q_{\hat{T}}, A_{s, t}$ is $\mathcal{G}_{t}^{s}$-measurable for every $0 \leq s \leq t \leq T$ and $P_{s, x}\left(A_{s, t}=A_{s, u}+\right.$ $\left.A_{u, t}, s \leq u \leq t \leq T\right)=1$ for quasi-every $(s, x) \in Q_{\hat{T}}$ (for the definition of exceptional sets see Section 5.1). If, in addition, $A_{s, \text { has }} P_{s, x}$-almost all continuous trajectories for q.e. $(s, x) \in Q_{\hat{T}}$ then $A$ is called a continuous $\mathrm{AF}(\mathrm{CAF})$, and if $A_{s,}$. is an increasing process under $P_{s, x}$ for q.e. $(s, x) \in Q_{\hat{T}}$, it is called a positive AF (PAF). If $M$ is an AF such that for q.e. $(s, x) \in Q_{\hat{T}}, E_{s, x}\left|M_{s, t}\right|^{2}<\infty$ and $E_{s, x} M_{s, t}=0$ for $t \in[s, T]$, it is called a martingale AF (MAF). Finally, we say that $A$ is an AF (CAF, PAF, MAF) in the strict sense if the corresponding property holds for every $(s, x) \in Q_{\hat{T}}$.

From [39, Theorem 2.1] it follows that there exist a strict MAF $M$ of $\mathbb{X}$ and a strict $\mathrm{CAF} A$ of $\mathbb{X}$ such that the quadratic variation $\left\langle A_{s,}\right\rangle_{T}$ of $A_{s, \text { on }}[s, T]$ equals zero $P_{s, x}$-a.s. and

$$
X_{t}-X_{s}=M_{s, t}+A_{s, t}, \quad t \in[s, T], \quad P_{s, x} \text {-a.s. }
$$

for every $(s, x) \in Q_{\hat{T}}$. In particular, $X$ is a $\left(\left\{\mathcal{G}_{t}^{s}\right\}, P_{s, x}\right)$-Dirichlet process on $[s, T]$ for every $(s, x) \in Q_{\hat{T}}$. Moreover, the above decomposition is unique and for every

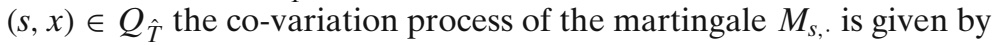

$$
\left\langle M_{s,,}^{i}, M_{s,}^{j}\right\rangle_{t}=\int_{s}^{t} a_{i j}\left(\theta, X_{\theta}\right) d \theta, \quad t \in[s, T], \quad i, j=1, \ldots, d
$$

(see [39] for details).

For $0 \leq s \leq u \leq t \leq T$ and $x \in \mathbb{R}^{d}$ we set

$$
\alpha_{u, t}^{s, x, i}=\frac{1}{2} \sum_{j=1}^{d} \int_{u}^{t} a_{i j}\left(\theta, X_{\theta}\right) p^{-1} \frac{\partial p}{\partial y_{j}}\left(s, x, \theta, X_{\theta}\right) d \theta, \quad \beta_{u, t}^{i}=\int_{u}^{t} b_{i}\left(\theta, X_{\theta}\right) d \theta .
$$

From [38] it follows that for each $(s, x) \in Q_{\hat{T}}$ the process $X$ admits under $P_{s, x}$ the following form of the Lyons-Zheng (see [23]) decomposition

$$
X_{t}-X_{u}=\frac{1}{2} M_{u, t}+\frac{1}{2}\left(N_{s, T+s-t}^{s, x}-N_{s, T+s-u}^{s, x}\right)-\alpha_{u, t}^{s, x}+\beta_{u, t}, \quad s \leq u \leq t \leq T,
$$

where $M_{s, \text { is }}$ is the martingale of Eq. 2.2 and $N_{s, \cdot}^{s, x}$ is a $\left(\left\{\overline{\mathcal{G}}_{t}^{s}\right\}, P_{s, x}\right)$-martingale such that

$$
\left\langle N_{s, \cdot, i}^{s, x, i}, N_{s, \cdot,}^{s, x, j}\right\rangle_{t}=\int_{s}^{t} a_{i j}\left(\bar{\theta}, \bar{X}_{\theta}\right) d \theta, \quad t \in[s, T], \quad i, j=1, \ldots, d .
$$

(In the sequel, for a process $Y$ on $[s, T]$ and fixed measure $P_{s, x}$ we write $\bar{Y}_{t}=$ $Y_{T+s-t}$ for $\left.t \in[s, T]\right)$.

Let $\mathbf{f}=\left(f^{1}, \ldots, f^{d}\right) \in C\left(Q_{T}\right)^{d}$. Similarly to [40, 43], for every $(s, x) \in Q_{\hat{T}}$ we put

$$
\int_{u}^{t} \mathbf{f}\left(\theta, X_{\theta}\right) d^{*} X_{\theta} \equiv-\int_{u}^{t} \mathbf{f}\left(\theta, X_{\theta}\right)\left(d M_{s, \theta}+d \alpha_{s, \theta}^{s, x}\right)-\int_{T+s-t}^{T+s-u} \mathbf{f}\left(\bar{\theta}, \bar{X}_{\theta}\right) d N_{s, \theta}^{s, x}
$$


for $s \leq u \leq t \leq T, P_{s, x}$-a.s.. The integrals on the right-hand side of the above expression are well defined under the measure $P_{s, x}$ for every $(s, x) \in Q_{\hat{T}}$ (see [16, Proposition 7.6])). Moreover, from [40] it follows that if $\mathbf{f} \in C_{c}^{\infty}\left(Q_{T}\right)^{d}$ then

$$
\int_{s}^{t} \mathbf{f}\left(\theta, X_{\theta}\right) d^{*} X_{\theta}=\int_{s}^{t} \operatorname{div}(a \mathbf{f})\left(\theta, X_{\theta}\right) d \theta, \quad s \leq t \leq T, \quad P_{s, x} \text {-a.s. }
$$

for every $(s, x) \in Q_{\hat{T}}$.

\subsection{Markov Type BSDEs}

In this section we consider BSDEs and RBSDEs with forward driving process $\left(X, P_{s, x}\right)$ and final conditions, generators and obstacles being functionals of $X$.

In what follows by $\mathcal{R}$ we denote the space of all functions $\varrho: \mathbb{R}^{d} \rightarrow \mathbb{R}$ of the form $\varrho(x)=\left(1+|x|^{2}\right)^{-\alpha}, x \in \mathbb{R}^{d}$, for some $\alpha \in \mathbb{R}$, and by $\mathcal{R}_{I}$ we denote the set of $\varrho \in \mathcal{R}$ such that $\int_{\mathbb{R}^{d}} \varrho(x) d x<\infty$. In the whole paper we assume that $\varrho \in \mathcal{R}_{I}$ unless it is explicitly stated otherwise.

Proposition 2.2 Let $\varrho \in \mathcal{R}$. Then there exist $0<c \leq C$ depending only on $\lambda, \Lambda$ and $\varrho$ such that for any $s \in[0, T)$ and $\psi \in \mathbb{L}_{1, \varrho}\left(Q_{s T}\right)$,

$$
\begin{aligned}
c \int_{t}^{T} \int_{\mathbb{R}^{d}}|\psi(\theta, x)| \varrho(x) d \theta d x & \leq \int_{t}^{T} \int_{\mathbb{R}^{d}} E_{s, x}\left|\psi\left(\theta, X_{\theta}\right)\right| \varrho(x) d \theta d x \\
& \leq C \int_{t}^{T} \int_{\mathbb{R}^{d}}|\psi(\theta, x)| \varrho(x) d \theta d x, \quad t \in[s, T] .
\end{aligned}
$$

Proof Follows from Proposition 5.1 in Appendix in [3] and Aronson's estimates (see $[2$, Theorem 7]).

Fix $(s, x) \in Q_{\hat{T}}$ and write

$$
B_{s, t}=\int_{s}^{t} \sigma^{-1}\left(\theta, X_{\theta}\right) d M_{s, \theta}, \quad t \in[s, T] .
$$

By Eq. 2.3, $\left\{B_{s, t}\right\}_{t \in[s, T]}$ is a $\left\{\mathcal{G}_{t}^{s}\right\}$-Wiener process under $P_{s, x}$. Moreover, from [21, Theorem 12] it follows that $B_{s,}$. has the representation property.

In what follows, in Sections 2-5, given $(s, x) \in Q_{\hat{T}}$ we consider the filtered probability space $\mathbb{G}_{s, x}=\left(\Omega, \mathcal{G},\left\{\mathcal{G}_{t}^{s}\right\}_{t \in[s, T]}, P_{s, x}\right)$ with the Wiener process $B_{s, .}$

By $\mathcal{D}^{2}(s, x, T), \mathcal{S}^{2}(s, x, T), \mathcal{M}^{2}(s, x, T), \mathcal{A}^{2}(s, x, T)$ we denote the spaces of processes $\mathcal{D}^{2}, \mathcal{S}^{2}, \mathcal{M}^{2}, \mathcal{A}^{2}$ (see Section 6) defined on $\mathbb{G}_{s, x}$. Thus, $\mathcal{D}^{2}(s, x, T)$ $\left(\mathcal{S}^{2}(s, x, T)\right)$ is the space of càdlàg (continuous) $\left\{\mathcal{G}_{t}^{s}\right\}$-progressively measurable processes $\eta$ on $[s, T]$ such that $E_{s, x} \sup _{s \leq t \leq T}\left|\eta_{t}\right|^{2}<\infty$ and $\mathcal{M}^{2}(s, x, T)\left(\mathcal{A}^{2}(s, x, T)\right)$ is the space of $\left\{\mathcal{G}_{t}^{s}\right\}$-progressively measurable (progressively measurable càdlàg increasing) processes such that $E_{s, x} \int_{s}^{T}\left|\eta_{t}\right|^{2}<\infty\left(E_{S, x}\left|\eta_{T}\right|^{2}<\infty\right)$.

For given $\xi, F, S$ satisfying (A1)-(A3), (A5) (resp. (A5*)) (see Section 6) on the space $\mathbb{G}_{s, x}$, by $\operatorname{BSDE}_{s, x}(\xi, F)$, (resp. $\left.\operatorname{RBSDE}_{s, x}(\xi, F, S), \overline{\operatorname{RBSDE}}_{s, x}(\xi, F, S)\right)$ we denote $\operatorname{BSDE}(\xi, F)$, (resp. $\underline{\operatorname{RBSDE}}(\xi, F, S), \overline{\operatorname{RBSDE}}(\xi, F, S))$ defined on $\mathbb{G}_{s, x}$ with the Wiener process $B_{s, \cdot}$ (definitions of solutions of general equations with data $\xi, F, S$ are given in Section 6). 
Furthermore, given $\varphi: \mathbb{R}^{d} \rightarrow \mathbb{R}, f: Q_{T} \times \mathbb{R} \times \mathbb{R}^{d} \rightarrow \mathbb{R}, h: Q_{T} \rightarrow \mathbb{R}$ we set

$$
\xi=\varphi\left(X_{T}\right), \quad F(t, \omega, y, z)=f\left(t, X_{t}(\omega), y, \sigma^{-1}\left(t, X_{t}(\omega)\right) z\right), \quad S_{t}=h\left(t, X_{t}\right)
$$

for $\omega \in \Omega, t \in[0, T]$, where $\sigma$ is the symmetric square-root of $a$ and $\sigma^{-1}$ is the inverse of $\sigma$, and by $\operatorname{BSDE}_{s, x}(\varphi, f)\left(\operatorname{resp} . \underline{\operatorname{RBSDE}}_{s, x}(\varphi, f, h), \overline{\operatorname{RBSDE}} \operatorname{BD}_{s, x}(\varphi, f, h)\right)$ we denote the $\operatorname{BSDE}_{s, x}(\xi, F)\left(\operatorname{resp} . \underline{\operatorname{RBSDE}}_{s, x}(\xi, F, S), \overline{\operatorname{RBSDE}}_{s, x}(\xi, F, S)\right)$.

Proposition 2.3 Assume that $\varphi, f$ satisfy $(\mathrm{H} 1)-(\mathrm{H} 3)$. Then for q.e. $(s, x) \in Q_{\hat{T}}$ there exists a unique solution of $\operatorname{BSDE}_{s, x}(\varphi, f)$. If, in addition, there exists $\phi \in \mathcal{P}$ such that $\phi \geq h^{+}$(resp. $\phi \leq h^{-}$) then for q.e. $(s, x) \in Q_{\hat{T}}$ there exists a unique solution of $\underline{R} B S D E_{s, x}(\varphi, f, h)\left(\operatorname{resp} . \bar{R} B S D E_{s, x}(\varphi, f, h)\right)$.

Proof It follows from Proposition 3.6 and Theorem 5.3 in [17].

In the case of two barriers $h_{1}, h_{2}: Q_{T} \rightarrow \mathbb{R}$ we set

$$
L_{t}=h_{1}\left(t, X_{t}\right), \quad U_{t}=h_{2}\left(t, X_{t}\right), \quad t \in[0, T] .
$$

Proposition 2.4 Assume that $(\mathrm{H} 1)-(\mathrm{H} 4)$ are satisfied. Then for q.e. $(s, x) \in Q_{\hat{T}}$ there exists a unique solution of $\operatorname{RBSDE}_{s, x}\left(\varphi, f, h_{1}, h_{2}\right)$ in the sense of the definition following condition (A6).

Proof Follows from [15, Theorem 3.7] and the fact that by Proposition 2.2 and [17, Remark 3.2], if (H1)-(H4) are satisfied then $\xi, F, L, U$ satisfy (A1)-(A4) and (A6) for q.e. $(s, x) \in Q_{\hat{T}}$.

Write $\mathcal{L}_{f} u=\frac{\partial u}{\partial t}+L_{t} u+f_{u}, \mathcal{L}=\mathcal{L}_{0}$ and set

$$
\begin{gathered}
\mathcal{P}_{f}=\left\{u \in \mathcal{F}: \mathcal{L}_{f} u \leq 0 \text { in } \mathcal{D}^{\prime}\left(\check{Q}_{T}\right), \underset{t \in[0, T]}{\operatorname{ess} \sup }\|u(t)\|_{H}<\infty\right\}, \quad \mathcal{P}=\mathcal{P}_{0}, \\
\overline{\mathcal{P}}_{f}=\left\{u \in \mathcal{F}: \mathcal{L}_{f} u \geq 0 \text { in } \mathcal{D}^{\prime}\left(\check{Q}_{T}\right), \underset{t \in[0, T]}{\operatorname{ess} \sup }\|u(t)\|_{H}<\infty\right\} .
\end{gathered}
$$

In what follows given $u \in \mathcal{P}_{f}$ we denote by $\mathcal{E}_{f}(u)$ a unique measure associated in the sense of [17, Theorem 4.6] with the potential $u$ such that $\varphi=\bar{u}(T-)$. If $f \equiv 0$ we write $\mathcal{E}(u)$ instead of $\mathcal{E}_{0}(u)$.

Proposition 2.5 Assume that $(\mathrm{H} 1)-(\mathrm{H} 3)$ and $(\mathrm{H} 4 *)$ are satisfied. Then for q.e. $(s, x) \in$ $Q_{\hat{T}}$ there exists a unique solution of $\operatorname{RBSDE}_{s, x}\left(\varphi, f, h_{1}, h_{2}\right)$ in the sense of the definition following condition (A6*).

Proof If (H1)-(H3) are satisfied then it follows from Proposition 2.2 and [17, Remark 3.2] that $\xi, F$ satisfy (A1)-(A4) for q.e. $(s, x) \in Q_{\hat{T}}$, and if (H4*) is satisfied then the obstacles $L, U$ satisfy $\left(\mathrm{A} 6^{*}\right)$ for q.e. $(s, x) \in Q_{\hat{T}}$. Indeed, let $\phi^{+}, \phi^{-} \in \mathcal{P}$, 
$\phi=\phi^{+}-\phi^{-}$. Then by [17, Theorem 5.3] there exist quasi-càdlàg versions $\bar{\phi}^{+}, \bar{\phi}^{-}$of $\phi^{+}$and $\phi^{-}$, respectively, such that for q.e. $(s, x) \in Q_{\hat{T}}$,

$$
\begin{aligned}
& \bar{\phi}^{+}\left(t, X_{t}\right)=\bar{\phi}^{+}(s, x)+\int_{s}^{t} d \mathcal{E}\left(\phi^{+}\right)\left(\theta, X_{\theta}\right)+\int_{s}^{t} \sigma \nabla \phi^{+}\left(\theta, X_{\theta}\right) d B_{s, \theta}, \\
& \bar{\phi}^{-}\left(t, X_{t}\right)=\bar{\phi}^{-}(s, x)+\int_{s}^{t} d \mathcal{E}\left(\phi^{-}\right)\left(\theta, X_{\theta}\right)+\int_{s}^{t} \sigma \nabla \phi^{-}\left(\theta, X_{\theta}\right) d B_{s, \theta}
\end{aligned}
$$

and

$$
\begin{gathered}
\int_{s} d \mathcal{E}\left(\phi^{+}\right)\left(\theta, X_{\theta}\right), \int_{s}^{\cdot} d \mathcal{E}\left(\phi^{-}\right)\left(\theta, X_{\theta}\right) \in \mathcal{A}^{2}(s, x, T), \\
\sigma \nabla \phi^{+}(\cdot, X .), \sigma \nabla \phi^{-}(\cdot, X .) \in \mathcal{M}^{2}(s, x, T),
\end{gathered}
$$

which shows that $\left(\mathrm{A} 6^{*}\right)$ is satisfied for q.e. $(s, x) \in Q_{\hat{T}}$. Therefore the desired result follows from [30, Theorem 2.3].

\subsection{Parabolic Capacity, Quasi-Continuity}

Definition The parabolic capacity of an open subset $B$ of $\check{Q}_{T}$ is given by

$$
\operatorname{cap}_{\mathcal{L}}(B)=\int_{0}^{T} P_{s, m}\left(\left\{\exists t \in(s, T):\left(t, X_{t}\right) \in B\right\}\right) d s,
$$

where $m$ is the Lebesgue measure on $\mathbb{R}^{d}$ and

$$
P_{s, m}(\Gamma)=\int_{\mathbb{R}^{d}} P_{s, x}(\Gamma) d x, \quad \Gamma \in \mathcal{G} .
$$

It is known (see Theorem A.1.2 and Lemmas A.2.5, A.2.6 in [13]) that this set function can be extended to a Choquet capacity on $\mathcal{B}\left(\check{Q}_{T}\right)$ in such a way that Eq. 2.6 holds for every compact set $K \subset \check{Q}_{T}$. We further extend this capacity to $\mathcal{B}\left(Q_{\hat{T}}\right)$ by putting $\operatorname{cap}_{\mathcal{L}}(\{0\} \times B)=m(B)$ for $B \in \mathcal{B}\left(\mathbb{R}^{d}\right)$.

Definition We say that $u: Q_{T} \rightarrow \mathbb{R}$ is quasi-continuous (quasi-càdlàg) if it is Borel measurable and the process $[s, T] \ni t \mapsto u\left(t, X_{t}\right)$ has continuous (càdlàg) trajectories under the measure $P_{s, x}$ for q.e. $(s, x) \in Q_{\hat{T}}$.

Definition Let $V \subset \check{Q}_{T}$ be an open set. The parabolic capacity of $V$ (in the analytical sense) is given by

$$
\operatorname{cap}_{2}(V)=\inf \left\{\|u\|_{\mathcal{W}_{\varrho}} ; u \in \mathcal{W}_{\varrho}, u \geq \mathbf{1}_{V} \text { a.e. }\right\}
$$

with the convention that $\inf \emptyset=\infty$. The parabolic capacity of a Borel subset $B$ of $\check{Q}_{T}$ is given by

$$
\operatorname{cap}_{2}(B)=\inf \left\{\operatorname{cap}_{2}(V) ; V \text { is an open subset of } \check{Q}_{T}, B \subset V\right\} .
$$




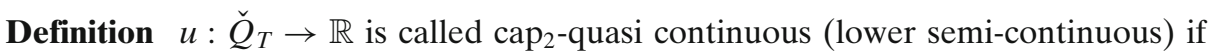
for every $\varepsilon>0$ there exists an open set $U_{\varepsilon} \subset \check{Q}_{T}$ such that $\operatorname{cap}_{2}\left(U_{\varepsilon}\right)<\varepsilon$ and $u_{\mid \check{Q}_{T} \backslash U_{\varepsilon}}$ is continuous (1.s.c.).

It is known (see, e.g., [26, 32]) that the capacities $\operatorname{cap}_{\mathcal{L}}$ and $\operatorname{cap}_{2}$ are equivalent. Moreover, by the same method as in the elliptic case (see Remark 3.6 and Proposition 3.7 in [42]) one can prove the following result.

Proposition 2.6 Let $u \in \mathcal{B}\left(\check{Q}_{T}\right)$. If $u$ is quasi-continuous then there exists $\bar{u}$ such that

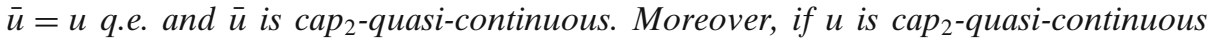
then $u$ is quasi-continuous.

Remark 2.7 It is known (see, e.g., [17, 26, 32]) that every $u \in \mathcal{W}_{\varrho}$ has a quasicontinuous version which is determined q.e..

\section{Nonhomogeneous Random Measures and Smooth Measures}

Let $\mu$ be a nonnegative Borel measure on $Q_{T}$ and let $t \in[0, T]$. By $\mu(t)$ we denote the Borel measure on $\mathbb{R}^{d}$ such that $\mu(t)(B)=\mu(\{t\} \times B)$ for $B \in \mathcal{B}\left(\mathbb{R}^{d}\right)$.

Definition We say that a nonnegative Borel measure $\mu$ on $Q_{T}$ is smooth if

(a) $\mu_{\mid \check{Q}_{T}} \ll \operatorname{cap}_{2}$

(b) There exists an increasing sequence $\left\{E_{n}\right\}$ of Borel subsets of $\check{Q}_{T}$ such that $\mu\left(E_{n}\right)<\infty$ for $n \in \mathbb{N}$ and $\operatorname{cap}_{2}\left(\breve{Q}_{T} \backslash \bigcup_{n} E_{n}\right)=0$,

(c) $\mu(0) \equiv 0$ and $\mu(T) \ll m$.

A sequence $\left\{E_{n}\right\}$ satisfying (b) without the condition $\mu\left(E_{n}\right)<\infty, n \in \mathbb{N}$, is called a weak nest, and with this condition a weak nest for $\mu$. We say that a family $\left\{F_{n}\right\}$ is a generalized nest if $\left\{F_{n}\right\}$ is an increasing sequence of closed subsets of $\check{Q}_{T}$ such that $\operatorname{cap}_{2}\left(K \backslash F_{n}\right) \rightarrow 0$ as $n \rightarrow+\infty$ for any compact subset $K \subset \check{Q}_{T}$. Finally, we say that a family $\left\{F_{n}\right\}$ is a nest if $\left\{F_{n}\right\}$ is an increasing sequence of closed subsets of $\check{Q}_{T}$ such that $\operatorname{cap}_{2}\left(\check{Q}_{T} \backslash F_{n}\right) \rightarrow 0$ as $n \rightarrow+\infty$. Of course every nest is a generalized nest.

Remark 3.1 A generalized nest is a weak nest. To see this, let us consider an increasing sequence $\left\{K_{n}\right\}$ of compact subsets of $\check{Q}_{T}$ such that $\bigcup_{n} K_{n}=\check{Q}_{T}$. By the definition of a generalized nest, for every $\varepsilon>0$ and $n \in \mathbb{N}$ there exists $m(n) \in \mathbb{N}$ such that $\operatorname{cap}_{2}\left(K_{n} \backslash F_{m(n)}\right)<2^{-n} \varepsilon$. Hence

$$
\operatorname{cap}_{2}\left(\check{Q}_{T} \backslash \bigcup_{m} F_{m}\right)=\operatorname{cap}_{2}\left(\bigcup_{n}\left(K_{n} \backslash \bigcup_{m} F_{m}\right)\right) \leq \sum_{n=1}^{+\infty} \operatorname{cap}_{2}\left(K_{n} \backslash F_{m(n)}\right)<\varepsilon
$$

for every $\varepsilon>0$, which shows that $\operatorname{cap}_{2}\left(\check{Q}_{T} \backslash \bigcup_{n} F_{n}\right)=0$.

In what follows by $S$ we denote the set of all smooth measures on $Q_{T}$. $S_{0}=\{\mu \in$ $\left.S ; \mu \in \mathcal{W}_{\varrho}^{\prime}\right\}$ and $\mathcal{M}_{0}$ stands for the set of Radon measures $\mu$ on $Q_{T}$ such that $\mu \in S$. 
From [32, Proposition 2] it follows that there exists a Choquet capacity $c_{0}$ with the property of strong subadditivity such that $c_{0}(B) \leq C\left(\operatorname{cap}_{2}(B)\right)^{2}$ for every $B \in$ $\mathcal{B}\left(\check{Q}_{T}\right)$, which when combined with [11, Proposition 2.19] implies that for any quasicontinuous $u \in \mathcal{W}_{\varrho}$ and $t>0$,

$$
c_{0}(\{|u|>t\}) \leq C t^{-2}\|u\|_{\mathcal{W}_{\varrho}}^{2} .
$$

Proposition 3.2 Let $\mu \in \mathcal{M}_{b}^{+}\left(\check{Q}_{T}\right)$. If there exists $C \geq 0$ such that $\mu(A) \leq C c_{0}(A)$ for $A \in \mathcal{B}\left(\check{Q}_{T}\right)$ then $\mu \in \mathcal{W}_{\varrho}^{\prime}$.

Proof Let $u \in \mathcal{W}_{\varrho}$ be quasi-continuous and let $A_{\varepsilon}=\{0<|u| \leq \varepsilon\}, \quad 0<\varepsilon<1$. By Eq. 3.1,

$$
\begin{aligned}
\int_{Q_{T}}|u| d \mu & \leq \mu\left(A_{\varepsilon}\right)+\mu(\varepsilon<|u| \leq 1)+\sum_{k=0}^{\infty} 2^{k+1} \mu\left(2^{k}<|u| \leq 2^{k+2}\right) \\
& \leq \mu\left(A_{\varepsilon}\right)+C\left(c_{0}(|u|>\varepsilon)+\sum_{k=0}^{\infty} 2^{k+1} c_{0}\left(|u|>2^{k}\right)\right) \\
& \leq \mu\left(A_{\varepsilon}\right)+C\left(\varepsilon^{-2}+4\right)\|u\|_{\mathcal{W}_{\ell}}^{2} .
\end{aligned}
$$

Since $\mu\left(A_{\varepsilon}\right) \rightarrow 0$ as $\varepsilon \rightarrow 0^{+}$, we conclude from the above that $\mu \in \mathcal{W}_{\varrho}^{\prime}$.

The following proposition is a parabolic analogue of [13, Theorem 2.2.4].

Proposition 3.3 A Borel measure $\mu$ on $\check{Q}_{T}$ is smooth iff there exists a weak nest $\left\{E_{n}\right\}$ such that $\mathbf{1}_{E_{n}} d \mu \in S_{0}$ for every $n \in \mathbb{N}$.

Proof It suffices to repeat the proof of [13, Theorem 2.2.4] with capacity Cap used in [13] replaced by capacity $c_{0}$.

Definition Let $\mu$ be a Borel measure on $Q_{T}$ and let $K$ be a PAF of $\mathbb{X}$. We say that $\mu$ corresponds to $K$ (or $K$ corresponds to $\mu$ ), and we write $\mu \sim K$, if for quasi-every $(s, x) \in Q_{\hat{T}}$,

$$
E_{s, x} \int_{s}^{T} f\left(t, X_{t}\right) d K_{s, t}=\int_{Q_{s} T} f(t, y) p(s, x, t, y) d \mu(t, y)
$$

for all $f \in \mathcal{B}^{+}\left(Q_{T}\right)$.

This is the sort of Revuz duality (see [34, 35]). It is easy to see that if $\mu \in S_{0}$ and $\mu \sim K$ then $E_{s, x} K_{s, T}<\infty$ for q.e $(s, x) \in Q_{\hat{T}}$. It follows (see, e.g., [6, 35]) that there exists at most one PAF $K$ such that $\mu \sim K$. In fact we have the following result.

Proposition 3.4 For every $\mu \in S$ there exists at most one PAF $K$ such that $\mu \sim K$.

Proof Follows from Proposition 3.3. 
Definition We say that $d K: \Omega \times \mathcal{B}([0, T]) \rightarrow \mathbb{R}$ is a random measure if

(a) $\forall \omega \in \Omega, d K(\omega)$ is a nonnegative measure on $\mathcal{B}([0, T])$,

(b) the mapping $\omega \rightarrow d K(\omega)$ is $\left(\mathcal{G}, \mathcal{B}\left(\mathcal{M}^{+}([0, T])\right)\right)$-measurable,

(c) $\forall 0 \leq s \leq t \leq T, \int_{s}^{t} d K_{\theta}$ is $\mathcal{G}_{t}^{s}$-measurable.

Notice that each random measure $d K$ determines PAF $K$ by the formula

$$
K_{s, t}=\int_{s}^{t} d K_{\theta}, \quad 0 \leq s \leq t \leq T .
$$

From now on we write $\mu \sim d K$ if $\mu \sim K$.

Proposition 3.5 For every $\mu \in S$ there exists a unique random measure $d K$ such that $\mu \sim d K$.

Proof By Proposition 3.3 there exists a weak nest $\left\{E_{n}\right\}$ such that $\mu_{n}=\mathbf{1}_{E_{n}} d \mu \in$ $S_{0}, n \in \mathbb{N}$. By [17, Corollary 4.17], for each $n \in \mathbb{N}$ there is a random measure $d K^{n}$ such that $\mu_{n} \sim d K^{n}$. Let us observe that $d K^{n} \leq d K^{m}$ if $n \leq m$, because by uniqueness, $\mathbf{1}_{E_{n}} d K^{m}=d K^{n}$. We define a random measure $d K$ as follows: $d K(\omega)=$ $\lim _{n \rightarrow+\infty} d K^{n}(\omega)$ if the limit exists in the space $\mathcal{M}^{+}([0, T])$, and $d K(\omega)=0$ otherwise. It is clear that $d K \sim \mu$.

The random measure $d K$ corresponding to $\mu \in S$ in the sense of Proposition 3.5 will be denoted by $d \mu(\cdot, X$.). With this notation, $\mu \sim d \mu(\cdot, X$.).

In the sequel we write $d \mu(\cdot, X$. $)=d \mu^{+}(\cdot, X$. $)-d \mu^{-}(\cdot, X$. $)$ if $\mu=\mu^{+}-\mu^{-}$is a signed measure such that $\mu^{+}, \mu^{-} \in S$.

Lemma 3.6 Assume that for q.e. $(s, x) \in Q_{\hat{T}}$ we are given a supermartingale $Y^{s, x} \in$ $\mathcal{S}^{2}(s, x, T)$ such that for some $u \in \mathcal{B}\left(Q_{T}\right), Y_{t}^{s, x}=u\left(t, X_{t}\right), P_{s, x}$-a.s. for $t \in[s, T]$. Then there exists a quasi-continuous version $\bar{u}$ of $u$ such that $Y_{t}^{s, x}=\bar{u}\left(t, X_{t}\right), t \in[s, T], P_{s, x^{-}}$ a.s. for q.e. $(s, x) \in Q_{\hat{T}}$.

Proof Since $Y^{s, x}$ is a supermartingale for q.e $(s, x) \in Q_{\hat{T}}$, it follows from the DoobMeyer decomposition theorem that for q.e $(s, x) \in Q_{\hat{T}}$ there exists an increasing process $K^{s, x}$ and a uniformly integrable martingale $M^{s, x}$ such that

$$
Y_{t}^{s, x}=Y_{T}^{s, x}+\int_{t}^{T} d K_{\theta}^{s, x}-M_{T}^{s, x}-M_{t}^{s, x}, \quad t \in[s, T], \quad P_{s, x} \text {-a.s.. }
$$

Moreover, since $Y^{s, x} \in \mathcal{S}^{2}(s, x, T)$, standard arguments (see, e.g., the proof of [12, Proposition 5.1]) show that $K^{s, x} \in \mathcal{A}_{c}^{2}(s, x, T), M^{s, x} \in \mathcal{S}^{2}(s, x, T)$. Since the Wiener process $B_{s, \text {, }}$, has the representation property with respect to $\left\{\mathcal{G}_{t}^{s}\right\}$, there exists $Z^{s, x} \in$ $\mathcal{M}^{2}(s, x, T)$ such that

$$
Y_{t}^{s, x}=Y_{T}^{s, x}+\int_{t}^{T} d K_{\theta}^{s, x}-\int_{t}^{T} Z_{\theta}^{s, x} d B_{s, \theta}, \quad t \in[s, T], \quad P_{s, x} \text {-a.s. }
$$

for q.e. $(s, x) \in Q_{\hat{T}}$. By [12],

$$
E_{s, x} \sup _{s \leq t \leq T}\left|Y_{t}^{s, x}-Y_{t}^{s, x, n}\right|^{2} \rightarrow 0
$$


for q.e. $(s, x) \in Q_{\hat{T}}$, where $\left(Y^{s, x, n}, Z^{s, x, n}\right) \in \mathcal{S}^{2}(s, x, T) \otimes \mathcal{M}^{2}(s, x, T)$ is a unique solution of the BSDE

$$
Y_{t}^{s, x, n}=Y_{T}^{s, x}+\int_{t}^{T} n\left(Y_{\theta}^{s, x, n}-Y_{\theta}^{s, x}\right)^{-} d \theta-\int_{t}^{T} Z^{s, x, n} d B_{s, \theta}, \quad t \in[s, T], \quad P_{s, x} \text {-a.s.. }
$$

Since by the assumptions of the lemma, $Y_{T}^{s, x}=u\left(T, X_{T}\right)$ and

$$
\int_{t}^{T}\left(Y_{\theta}^{s, x}-Y_{\theta}^{s, x, n}\right)^{-} d \theta=\int_{t}^{T}\left(Y_{\theta}^{s, x}-u\left(\theta, X_{\theta}\right)\right)^{-} d \theta, \quad t \in[s, T], \quad P_{s, x^{-}} \text {a.s. }
$$

for q.e $(s, x) \in Q_{\hat{T}}$, it follows from [17, Proposition 3.6] that for q.e. $(s, x) \in Q_{\hat{T}}$,

$$
\left(Y^{s, x, n}, Z^{s, x, n}\right)=\left(\bar{u}_{n}(\cdot, X .), \sigma \nabla u_{n}(\cdot, X .)\right)
$$

in $\mathcal{S}^{2}(s, x, T) \otimes \mathcal{M}^{2}(s, x, T)$, where $\bar{u}_{n}$ is a quasi-continuous version of a unique solution of $\operatorname{PDE}\left(u(T), f_{n}\right)$ with $f_{n}(t, x, y)=n(y-u(t, x))^{-}$. We put $\bar{u}(s, x)=$ $\lim _{n \rightarrow+\infty} \bar{u}_{n}(s, x)$ if the limit exists, and $\bar{u}(s, x)=0$ otherwise. From Eq. 3.4 it follows that $\bar{u}$ has the desired properties.

Lemma 3.7 Let $K$ be a PCAF of $\mathbb{X}$. Then for every $\alpha>0$ the function $\phi_{\alpha}: Q_{\hat{T}} \rightarrow \mathbb{R}$ defined by

$$
\phi_{\alpha}(s, x)=E_{s, x} \int_{s}^{T} e^{-\alpha K_{s, t}} d t, \quad(s, x) \in Q_{\hat{T}}
$$

is quasi-continuous.

Proof Let $K^{n}=K \wedge n$. From [33] it follows that for q.e. $(s, x) \in Q_{\hat{T}}$ there exists a unique solution $\left(Y^{s, x, n}, Z^{s, x, n}\right) \in \mathcal{S}^{2}(s, x, T) \otimes \mathcal{M}^{2}(s, x, T)$ of the BSDE

$$
Y_{t}^{s, x, n}=\int_{t}^{T} d \theta-\alpha \int_{t}^{T} Y_{\theta}^{s, x, n} d K_{s, \theta}^{n}-\int_{t}^{T} Z_{\theta}^{s, x, n} d B_{s, \theta}, \quad t \in[s, T], \quad P_{s . x} \text {-a.s.. }
$$

Applying Itô's formula to $e^{-\alpha K_{s, \cdot}^{n}} Y^{s, x, n}$ we get

$$
Y_{t}^{s, x, n}=E_{s, x}\left(\int_{t}^{T} e^{-\alpha K_{t, \theta}^{n}} d \theta \mid \mathcal{G}_{t}^{s}\right), \quad P_{s, x} \text {-a.s., } \quad t \in[s, T] .
$$

Using [29, Theorem 2.1] we deduce from the above that there exists $\left(Y^{s, x}, Z^{s, x}\right) \in$ $\mathcal{S}^{2}(s, x, T) \otimes \mathcal{M}^{2}(s, x, T)$ such that

$$
Y_{t}^{s, x}=\int_{t}^{T} d \theta-\alpha \int_{t}^{T} Y_{\theta}^{s, x} d K_{s, \theta}-\int_{t}^{T} Z_{\theta}^{s, x} d B_{s, \theta}, \quad t \in[s, T], \quad P_{s . x} \text {-a.s. }
$$

and

$$
Y_{t}^{s, x}=E_{s, x}\left(\int_{t}^{T} e^{-\alpha K_{t, \theta}} d \theta \mid \mathcal{G}_{t}^{s}\right), \quad P_{s, x} \text {-a.s., } \quad t \in[s, T]
$$

for q.e. $(s, x) \in Q_{\hat{T}}$. From Eq. 3.5 and the Markov property it follows that there exists $u \in \mathcal{B}\left(Q_{T}\right)$ such that for q.e. $(s, x) \in Q_{\hat{T}}, Y_{t}^{s, x}=u\left(t, X_{t}\right), P_{s, x}$-a.s. for $t \in[s, T]$. By Lemma 3.6, $u$ has a quasi-continuous version $\bar{u}$ such that $Y_{t}^{s, x}=\bar{u}\left(t, X_{t}\right), t \in[s, T]$, $P_{s, x}$-a.s. for q.e. $(s, x) \in Q_{\hat{T}}$. Since $\bar{u}(s, x)=E_{s, x}\left(\int_{s}^{T} e^{-\alpha K_{s, \theta}} d \theta\right)$ for q.e. $(s, x) \in Q_{\hat{T}}$, the result follows. 


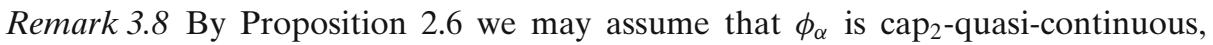
which implies that there is a nest $\left\{E_{n}\right\}$ for $\phi_{\alpha}$, that is $\left\{E_{n}\right\}$ is a nest and $u_{\mid E_{n}}$ is continuous for every $n \in \mathbb{N}$.

\section{Lemma 3.9 Let $K$ be a PCAF.}

(i) For q.e. $(s, x) \in Q_{\hat{T}}, E_{s, x} \int_{s}^{T} \phi_{\alpha}\left(t, X_{t}\right) d K_{s, t} \leq \frac{T-s}{\alpha}$ for all $\alpha>0$.

(ii) Let $\left\{E_{n}\right\}$ be a nest for $\phi_{1}$ and let $F_{n}=\left\{(s, x) \in E_{n} ; \phi_{1}(s, x) \geq \frac{1}{n}\right\}$. Then $\left\{F_{n}\right\}$ is a generalized nest.

Proof (i) For q.e. $(s, x) \in Q_{\hat{T}}$ we have

$$
\begin{aligned}
E_{s, x} & \int_{s}^{T} \phi_{\alpha}\left(t, X_{t}\right) d K_{s, t}=E_{s, x} \int_{s}^{T}\left(E_{t, X_{t}} \int_{t}^{T} e^{-\alpha K_{t, \theta}} d \theta\right) d K_{s, t} \\
= & E_{s, x} \int_{s}^{T} E_{s, x}\left(\int_{t}^{T} e^{-\alpha K_{t, \theta}} d \theta \mid \mathcal{G}_{t}^{s}\right) d K_{s, t}=E_{s, x} \int_{s}^{T}\left(\int_{s}^{\theta} e^{-\alpha K_{t, \theta}} d K_{s, t}\right) d \theta \\
= & E_{s, x} \int_{s}^{T} e^{-\alpha K_{s, \theta}}\left(\int_{s}^{\theta} e^{\alpha K_{s, t}} d K_{s, t}\right) d \theta=E_{s, x} \int_{s}^{T} e^{-\alpha K_{s, \theta}} \frac{1}{\alpha} \int_{s}^{\theta} d\left(e^{\alpha K_{s, t}}\right) \\
= & \frac{1}{\alpha} E_{s, x} \int_{s}^{T} e^{-\alpha K_{s, \theta}}\left(e^{\alpha K_{s, \theta}}-1\right) d \theta=\frac{1}{\alpha} E_{s, x} \int_{s}^{T}\left(1-e^{-\alpha K_{s, \theta}}\right) d \theta \leq \frac{T-s}{\alpha} .
\end{aligned}
$$

To prove (ii) we put $N=\bigcap_{n=1}^{\infty}\left(\check{Q}_{T} \backslash E_{n}\right), B_{n}=\left\{(s, x) \in \check{Q}_{T} \backslash N ; \phi_{\alpha}(x) \leq \frac{1}{n}\right\}$ and $\sigma_{n}=\sigma_{B_{n}}, \sigma=\lim _{n \rightarrow \infty} \sigma_{n}$. By the quasi-continuity of $\phi_{\alpha}$, the Markov property and Lemma 3.7,

$$
E_{s, x} \int_{\sigma_{n}}^{T} e^{-\alpha K_{s, t}} d t=E_{s, x} e^{-\alpha K_{s, \sigma_{n}}} \phi_{\alpha}\left(\sigma_{n}, X_{\sigma_{n}}\right) \leq \frac{1}{n} .
$$

Hence $P_{s, x}(\sigma<T)=0$ for q.e. $(s, x) \in Q_{\hat{T}}$. Since $\check{Q}_{T} \backslash F_{n} \subset\left(\check{Q}_{T}-E_{n}\right) \cup B_{n}$, it follows from [26, Lemma 6.1] that $\left\{F_{n}\right\}$ is a generalized nest. and

By $\mathcal{A}_{\varrho}^{2}$ we denote the set of PAFs $K$ such that $K_{s, .} \in \mathcal{A}^{2}(s, x, T)$ for q.e. $(s, x) \in Q_{\hat{T}}$

$$
\sup _{s \in[0, T)} \int_{\mathbb{R}^{d}}\left(E_{s, x} K_{s, T}^{2}\right) \varrho^{2}(x) d x<\infty
$$

Proposition 3.10 If $K \in \mathcal{A}_{\varrho}^{2}$ then there exists a unique measure $\mu \in S_{0}$ such that $\mu \sim K$.

Proof Let $\left(Y^{s, x}, Z^{s, x}\right)$ be a unique solution of $\operatorname{BSDE}_{s, x}(\varphi, K)$. By the Markov property, $Y_{t}^{s, x}=v\left(t, X_{t}\right), P_{s, x}$-a.s., $t \in[s, T]$ for q.e. $(s, x) \in Q_{\hat{T}}$, where $v(s, x)=Y_{s}^{s, x}$. Hence the triple $\left(Y^{s, x}, Z^{s, x}, K\right)$ is a unique solution of $\operatorname{BSDE}_{s, x}(\varphi, 0, v)$. By [17, Theorem 5.3], there exists a unique solution $(u, \mu)$ of $\underline{\mathrm{OP}}(\varphi, 0, v)$ (for the definition of the last problem see Section 5.1) such that $u(\cdot, \bar{X})=.Y^{s, x}$ in $\mathcal{D}^{2}(s, x, T)$ and $d \mu(\cdot, X$. $)=d K$ q.e..

Proposition 3.11 Any PCAF $K$ admits a measure $\mu \in S$ such that $\mu \sim K$. 
Proof Write $d K_{s, t}^{\alpha}=\phi_{\alpha}\left(t, X_{t}\right) d K_{s, t}$. By [7], for each $\alpha>0$ there exists a unique solution $\left(Y^{s, x, \alpha}, Z^{s, x, \alpha}\right)$ of $\operatorname{BSDE}_{s, x}\left(0, K^{\alpha}\right)$. By Lemma 3.9, $E_{s, x} Y_{t}^{s, x} \leq \frac{T}{\alpha}$. On the other hand, by the Markov property, $Y_{t}^{s, x, \alpha}=u_{\alpha}\left(t, X_{t}\right), P_{s, x}$-a.s. for every $t \in[s, T]$, where $u_{\alpha}(s, x)=Y_{s}^{s, x, \alpha}$. Hence $\left|Y_{t}^{s, x, \alpha}\right| \leq \frac{T}{\alpha}, s \leq t \leq T, P_{s, x}$-a.s. for q.e. $(s, x) \in Q_{\hat{T}}$. Using this, Itô's formula and the Burkholder-Davis-Gundy inequality one can show that for q.e. $(s, x) \in Q_{\hat{T}}$,

$$
E_{s, x}\left|K_{s, T}^{\alpha}\right|^{2}+E_{s, x} \int_{s}^{T}\left|Z_{t}^{s, x, \alpha}\right|^{2} d t \leq \frac{T}{\alpha} .
$$

By Proposition 3.10, there exists a measure $\mu_{\alpha} \in S_{0}$ such that $\mu_{\alpha} \sim K^{\alpha}$. Since $K^{\alpha} \nearrow K$ as $\alpha \downarrow 0$, for every $B \in \mathcal{B}\left(Q_{T}\right)$ the sequence $\left\{\mu_{\alpha}(B)\right\}$ is increasing and hence convergent. By the Hahn-Saks theorem, there exists a measure $\mu$ such that $\mu_{\alpha}(B) \rightarrow \mu(B)$ for every $B \in \mathcal{B}\left(Q_{T}\right)$. Of course, $\mu_{\mid \check{o}_{T}} \ll \operatorname{cap}_{\mathcal{L}}, \mu(0) \equiv 0, \mu(T) \ll m$ and $\mu \sim K$. We now show that $\left\{F_{n}\right\}$ defined in Lemma 3.9 is a generalized nest for $\mu$. By Lemma 3.9, for a.e. $x \in \mathbb{R}^{d}$ we have

$$
\begin{aligned}
\int_{Q_{T}} \mathbf{1}_{F_{n}}(t, y) p(0, x, t, y) d \mu(t, y) & =E_{0, x} \int_{0}^{T} \mathbf{1}_{F_{n}}\left(t, X_{t}\right) d K_{0, t} \\
& \leq n E_{0, x} \int_{0}^{T} \phi_{1}\left(t, X_{t}\right) d K_{0, t} \leq T n .
\end{aligned}
$$

Integrating the above inequality with respect to the measure $\varrho(x) m(d x)$ and using Aronson's estimate on $p$ we see that $\mu\left(F_{n}\right)<\infty$, which when combined with Lemma 3.9(ii) and the already proven properties of $\mu$ shows that $\mu \in S$.

\section{The Sobolev Space $W^{0,1}(\mathbb{X})$}

For the purposes of Section 5 in this section we introduce some stochastic generalization of the Sobolev space $W_{2, \varrho}^{0,1}$ and prove its basic properties.

Lemma 4.1 For any $\alpha>0$ and $(s, x) \in Q_{\hat{T}}$,

$$
\int_{s}^{T} \frac{|D p|^{2}}{p^{2}}\left(s, x, t, X_{t}\right)(t-s)^{\alpha} d t<\infty, \quad P_{s, x} \text {-a.s.. }
$$

Proof Follows immediately from [37, Lemma 5.2].

We write $u \in \mathcal{D}^{2}$ (resp. $u \in \mathcal{S}^{2}$ ) if $u$ is a Borel measurable function on $Q_{T}$ such that $u\left(\cdot, X\right.$.) $\in \mathcal{D}^{2}(s, x, T)\left(\right.$ resp. $u\left(\cdot, X\right.$.) $\left.\in \mathcal{S}^{2}\right)$ for q.e. $(s, x) \in Q_{\hat{T}}$. By $\mathcal{D}_{\varrho}^{2}\left(\right.$ resp. $\left.\mathcal{S}_{\varrho}^{2}\right)$ we denote the space of all functions $u$ from $\mathcal{D}^{2}$ (resp. $\mathcal{S}^{2}$ ) such that

$$
\sup _{s \in[0, T)} \int_{\mathbb{R}^{d}}\left(E_{s, x} \sup _{s \leq t \leq T}\left|u\left(t, X_{t}\right)\right|^{2}\right) \varrho^{2}(x) d x<\infty .
$$

$D([0, T] ; H)(\operatorname{resp} . C([0, T] ; H))$ is the space of functions $u \in \mathcal{B}\left(Q_{T}\right)$ such that the mapping $[0, T] \ni t \rightarrow u(t) \in H$ is càdlàg (continuous).

Proposition 4.2 $\mathcal{D}_{\varrho}^{2} \subset D([0, T] ; H)$. 
Proof Let $u \in \mathcal{D}_{\varrho}^{2}$. Fix $t_{0} \in[0, T), 0 \leq s \leq t_{0}$ and let $\left(X, Q_{s, x}\right)$ be diffusion associated with the operator $A_{t}$. From the fact that $u \in \mathcal{D}_{\varrho}^{2}$ it follows that for q.e. $(s, x) \in Q_{\hat{T}}$,

$$
\lim _{t \rightarrow t_{0}^{+}} E_{Q_{s, x}} u\left(t, X_{t}\right) \eta\left(X_{t}\right)=E_{Q_{s, x}} u\left(t_{0}, X_{t_{0}}\right) \eta\left(X_{t_{0}}\right), \quad \eta \in C_{c}\left(Q_{T}\right) .
$$

Applying the Lebesgue dominated convergence theorem we conclude from Eq. 4.1 and Remark 2.1 that $(u(t), \eta) \rightarrow\left(u\left(t_{0}\right), \eta\right)$ for any $\eta \in C_{c}\left(Q_{T}\right)$, hence that $u(t) \rightarrow$ $u\left(t_{0}\right)$ weakly in $H$ as $t \rightarrow t_{0}^{+}$since $\sup _{t \in[0, T]}\|u(t)\|_{H}<\infty$. Put $u_{k}(t)=T_{k}(u(t))$. Applying once again the Lebesgue dominated convergence theorem and using Eq. 4.1, boundedness of $u_{k}$ and the fact that $u \in \mathcal{D}_{\varrho}^{2}$ we get $\lim \sup _{t \rightarrow t_{0}^{+}}\left\|u_{k}(t)\right\|_{H}^{2} \leq\left\|u_{k}\left(t_{0}\right)\right\|_{H}^{2}$. Since the sequence $\left\{\left\|u_{k}(t)\right\|_{H}^{2}\right\}_{k \geq 0}$ is nondecreasing, letting $k \rightarrow \infty$ in the last inequality shows that $\limsup _{t \rightarrow t_{0}^{+}}\|u(t)\|_{H}^{2} \leq\left\|u\left(t_{0}\right)\right\|_{H}^{2}$. Therefore in fact $u(t) \rightarrow u\left(t_{0}\right)$ strongly in $H$ as $t \rightarrow t_{0}^{+}$because $H$ is a Hilbert space. Now, fix $t_{0} \in(0, T]$ and let $0 \leq s<t_{0}$. From [14, Theorem 16.4] it follows that there exists $\xi \in H$ such that $u_{-}\left(t_{0}, X_{t_{0}}\right) \equiv \lim _{t \rightarrow t_{0}^{-}} u\left(t, X_{t}\right)=\xi\left(X_{t_{0}}\right), P_{s, x}$-a.s. for a.e. $x \in \mathbb{R}^{d}$. Repeating previous arguments with $t \rightarrow t_{0}^{+}$replaced by $t \rightarrow t_{0}^{-}$one can show that $u(t) \rightarrow \xi$ strongly in $H$ as $t \rightarrow t_{0}^{-}$, which completes the proof of the proposition.

Note that from [17, Theorem 4.6] it follows that $\mathcal{P}_{f}, \overline{\mathcal{P}}_{f} \subset \mathcal{D}_{\varrho}^{2}$. Thus, $\mathcal{P}_{f}, \overline{\mathcal{P}}_{f} \subset$ $D([0, T] ; H)$.

Proposition 4.3 If $u \in \mathcal{W}_{\varrho}$ then

$$
\sup _{s \in[0, T)} \int_{\mathbb{R}^{d}}\left(E_{s, x} \sup _{s \leq t \leq T}\left|u\left(t, X_{t}\right)\right|^{2}\right) \varrho^{2}(x) d x \leq C\|u\|_{\mathcal{W}_{e}}^{2} .
$$

Proof By [32, Proposition 3] there exists $v \in \mathcal{P}$ such that $|u| \leq v$ a.e. and $\|v\|_{\mathcal{P}} \leq$ $C\|u\|_{\mathcal{W}_{\varrho}}$, where

$$
\|v\|_{\mathcal{P}}=\operatorname{ess}_{t \in[0, T]}\|v(t)\|_{H}+\|\nabla v\|_{\mathcal{H}}
$$

On the other hand, by [17, Theorem 4.6],

$$
\sup _{s \in[0, T)} \int_{\mathbb{R}^{d}}\left(E_{s, x} \sup _{s \leq t \leq T}\left|v\left(t, X_{t}\right)\right|^{2}\right) \varrho^{2}(x) d x \leq C\|v\|_{\mathcal{P}}^{2} .
$$

Since

$$
\int_{\mathbb{R}^{d}}\left(E_{s, x} \sup _{s \leq t \leq T}\left|u\left(t, X_{t}\right)\right|^{2}\right) \varrho^{2}(x) d x \leq \int_{\mathbb{R}^{d}}\left(E_{s, x} \sup _{s \leq t \leq T}\left|v\left(t, X_{t}\right)\right|^{2}\right) \varrho^{2}(x) d x
$$

(the expressions on both sides of the above inequality do not depend on versions of $u, v$, see (4.7) in [17]), the desired result follows.

Corollary 4.4 The embeddings $\mathcal{W}_{\varrho} \subset \mathcal{S}_{\varrho}^{2} \subset C([0, T] ; H)$ are continuous.

Proof Follows directly from Propositions 4.2 and 4.3.

We write $u \in \mathcal{M}$ if $u\left(\cdot, X\right.$.) $\in \mathcal{M}(s, x, T)$ for q.e. $(s, x) \in Q_{\hat{T}}$. 
Let $W^{0,1}(\mathbb{X})$ denote the set of all $u \in \mathcal{M}$ for which there exists a sequence $\left\{u_{n}\right\} \subset$ $C_{c}^{\infty}\left(Q_{T}\right)$ such that for q.e. $(s, x) \in Q_{\hat{T}}$,

$$
\int_{s}^{T}\left|\left(u_{n}-u\right)\right|^{2}\left(t, X_{t}\right) d t \rightarrow 0 \text { as } n \rightarrow \infty \text { in probability } P_{s, x}
$$

and

$$
\int_{s}^{T}\left|\nabla\left(u_{n}-u_{m}\right)\right|^{2}\left(t, X_{t}\right) d t \rightarrow 0 \text { as } n, m \rightarrow \infty \text { in probability } P_{s, x} .
$$

Let us mention that $\mathcal{F} \subset W^{0,1}(\mathbb{X})$. Indeed, if $u \in \mathcal{F}$ then there is a sequence $\left\{u_{n}\right\} \subset$ $C_{c}^{\infty}\left(Q_{T}\right)$ such that $\left\|u_{n}-u\right\|_{\mathcal{H}} \rightarrow 0$ as $n \rightarrow \infty$ and $\left\|\nabla u_{n}-\nabla u_{m}\right\|_{\mathcal{H}} \rightarrow 0$ as $n, m \rightarrow \infty$. From this and Proposition 2.2 we conclude that

$$
\begin{gathered}
\int_{Q_{T}}\left(E_{s, x} \int_{s}^{T}\left|\nabla u_{n}-\nabla u_{m}\right|^{2}\left(t, X_{t}\right) d t\right) \varrho^{2}(x) d s d x \\
\quad+\int_{Q_{T}}\left(E_{s, x} \int_{s}^{T}\left|u_{n}-u\right|^{2}\left(t, X_{t}\right) d t\right) \varrho^{2}(x) d s d x \\
\leq C\left(\left\|\nabla u_{n}-\nabla u_{m}\right\|_{\mathcal{H}}^{2}+\left\|u_{n}-u\right\|_{\mathcal{H}}^{2}\right),
\end{gathered}
$$

which implies that Eqs. 4.2, 4.3 hold along some subsequence (see [16, Proposition 3.3]).

Proposition 4.5 If $u \in W^{0,1}(\mathbb{X})$ then there exists a unique (in the almost everywhere sense) function $v \in \mathcal{B}\left(Q_{T}\right)$ such that for every $\left\{u_{n}\right\} \subset C_{c}^{\infty}\left(Q_{T}\right)$ satisfying Eqs. 4.2, 4.3,

$$
\int_{s}^{T}\left|\nabla u_{n}\left(t, X_{t}\right)-v\left(t, X_{t}\right)\right|^{2} d t \rightarrow 0 \text { as } n \rightarrow \infty \text { in probability } P_{s, x}
$$

for q.e. $(s, x) \in Q_{\hat{T}}$.

Proof Existence. Since $\mathbb{R}^{+} \ni x \rightarrow x \wedge 1$ is concave, it follows from Proposition 2.2 that

$$
\left\|\left|\nabla\left(u_{n}-u_{m}\right)\right| \wedge k\right\|_{\mathcal{H}}^{2} \leq C \int_{Q_{T}}\left(E_{s, x}\left(\int_{s}^{T}\left|\nabla\left(u_{n}-u_{m}\right)\right|^{2}\left(t, X_{t}\right) d t\right) \wedge k\right) \varrho^{2}(x) d x d s .
$$

Since $\varrho \in \mathcal{R}_{I}$, applying the Lebesgue dominated convergence theorem we conclude that the right-hand side of the above inequality tends to zero as $n, m \rightarrow \infty$. Therefore there is $v \in \mathcal{B}\left(Q_{T}\right)$ such that $\nabla u_{n} \rightarrow v$ in measure $\varrho^{2} d m_{T}$. Moreover,

$$
\int_{Q_{T}}\left(E_{s, x} \int_{s}^{T}\left(\left|\nabla\left(u_{n}-v\right)\left(t, X_{t}\right)\right|^{2} \wedge k\right) d t\right) \varrho^{2}(x) d x d s \leq C\left\|\left|\nabla\left(u_{n}-v\right)\right| \wedge k\right\|_{\mathcal{H}}^{2} \rightarrow 0
$$

as $n, m \rightarrow \infty$. Hence there is a subsequence such that if $n, m \rightarrow \infty$ along this subsequence then $E_{s, x} \int_{s}^{T}\left(\left|\nabla\left(u_{n}-v\right)\left(t, X_{t}\right)\right|^{2} \wedge k\right) d t \rightarrow 0$ for q.e. $(s, x) \in Q_{\hat{T}}$. On the other hand, from Eq. 4.3 it follows that for q.e. $(s, x) \in Q_{\hat{T}}$ there exists a process $Y^{s, x}$ such that

$$
\int_{s}^{T}\left|\nabla u_{n}\left(t, X_{t}\right)-Y_{t}^{s, x}\right|^{2} d t \rightarrow 0 \text { in probability } P_{s, x}
$$


and hence that $\int_{s}^{T}\left(\left|\nabla u_{n}\left(t, X_{t}\right)-Y_{t}^{s, x}\right|^{2} \wedge k\right) d t \rightarrow 0$ in probability $P_{s, x}$. By the Lebesgue dominated convergence theorem, $E_{s, x} \int_{s}^{T}\left(\left|\nabla u_{n}\left(t, X_{t}\right)-Y_{t}^{s, x}\right|^{2} \wedge k\right) d t \rightarrow 0$ as $n \rightarrow \infty$. Hence

$$
\begin{aligned}
E_{s, x} \int_{s}^{T}\left(\left|v\left(t, X_{t}\right)-Y_{t}^{s, x}\right| \wedge k\right) d t \leq & \lim _{n \rightarrow \infty} E_{s, x} \int_{s}^{T}\left(\left|v\left(t, X_{t}\right)-\nabla u_{n}\left(t, X_{t}\right)\right| \wedge k\right) d t \\
& +\lim _{n \rightarrow \infty} E_{s, x} \int_{s}^{T}\left(\left|\nabla u_{n}\left(t, X_{t}\right)-Y_{t}^{s, x}\right| \wedge k\right) d t=0
\end{aligned}
$$

for q.e. $(s, x) \in Q_{\hat{T}}$. By Fatou's lemma,

$$
E_{s, x} \int_{s}^{T}\left|v\left(t, X_{t}\right)-Y_{t}^{s, x}\right| d t=0
$$

and the proof is complete.

Uniqueness. Let $\left\{u_{n}\right\},\left\{\tilde{u}_{n}\right\} \subset C_{c}^{\infty}\left(Q_{T}\right)$ be such that Eqs. 4.2, 4.3 are satisfied. Then from the first part of the proof it follows that there exist $v, \tilde{v}$ such that Eq. 4.4 is satisfied. Let $\alpha>0, \eta \in C_{c}^{\infty}\left(Q_{T}\right)$ and let $\eta_{s} \in C_{c}^{\infty}\left(Q_{T}\right)$ be such that $\left(\eta_{s}\right)_{\mid Q_{s T}}=(\cdot-$ $s)^{\alpha} \eta$. Then from the identity

$$
\nabla u_{n} \eta_{s}=\operatorname{div}\left(u_{n} \eta_{s}\right)-u_{n} \operatorname{div} \eta_{s}
$$

and Eq. 2.4 it follows that for every $(s, x) \in Q_{\hat{T}}$,

$$
\begin{aligned}
\int_{t}^{T} \nabla u_{n} \eta_{s}\left(\theta, X_{\theta}\right) d \theta= & \int_{t}^{T} a^{-1}\left(\theta, X_{\theta}\right) u_{n}\left(\theta, X_{\theta}\right) \eta_{s}\left(\theta, X_{\theta}\right) d^{*} X_{\theta} \\
& -\int_{t}^{T} u_{n}\left(\theta, X_{\theta}\right) \operatorname{div} \eta_{s}\left(\theta, X_{\theta}\right) d \theta, \quad t \in[s, T], \quad P_{s, x} \text {-a.s.. }
\end{aligned}
$$

The same holds true with $u_{n}$ replaced by $\tilde{u}_{n}$. In view of Eqs. 4.2, 4.3 and Lemma 4.1 we may pass to the limit in the above equation and similar equation for $\tilde{u}_{n}$. We then get

$$
\int_{t}^{T} v\left(\theta, X_{\theta}\right) \eta_{s}\left(\theta, X_{\theta}\right) d \theta=\int_{t}^{T} \tilde{v}\left(\theta, X_{\theta}\right) \eta_{s}\left(\theta, X_{\theta}\right) d \theta, \quad t \in[s, T], \quad P_{s, x} \text {-a.s. }
$$

for q.e. $(s, x) \in Q_{\hat{T}}$. Since the above equality holds true for any $\eta \in C_{c}^{\infty}\left(Q_{T}\right)$, $v=\tilde{v}$ a.e..

Given $u \in W^{0,1}(\mathbb{X})$ we denote $v$ of Proposition 4.5 by $\nabla_{\mathbb{X}} u$. From the construction of $\nabla_{\mathbb{X}} u$ we see that if $u \in \mathcal{F}$ then $\nabla_{\mathbb{X}} u=\nabla u$ a.e..

Definition Let $u_{n}, u \in W^{0,1}(\mathbb{X})$. We say that $u_{n} \rightarrow u$ in $W^{0,1}(\mathbb{X})$ if

$$
\int_{s}^{T}\left|\left(u_{n}-u\right)\left(t, X_{t}\right)\right|^{2} d t \rightarrow 0 \text { in probability } P_{s, x}, \text { as } n \rightarrow \infty
$$

and

$$
\int_{s}^{T}\left|\left(\nabla_{\mathbb{X}} u_{n}-\nabla_{\mathbb{X}} u\right)\left(t, X_{t}\right)\right|^{2} d t \rightarrow 0 \text { in probability } P_{s, x} \text { as } n, m \rightarrow \infty
$$

for q.e. $(s, x) \in Q_{\hat{T}}$. 
Proposition 4.6 The space $W^{0,1}(\mathbb{X})$ with the topology of convergence defined by Eqs. 4.5, 4.6 is complete.

Proof Suppose that $\left\{u_{n}\right\} \subset W^{0,1}(\mathbb{X})$ and $u_{n}-u_{m} \rightarrow 0$ in $W^{0,1}(\mathbb{X})$ as $n, m \rightarrow+\infty$. Then as in the proof of Proposition 4.5 one can show that there exist functions $u, v \in \mathcal{B}\left(Q_{T}\right)$ such that $u_{n} \rightarrow u, \nabla_{\mathbb{X}} u_{n} \rightarrow v$ in measure $\varrho^{2} d m_{T}$ and Eqs. 4.5, 4.6 hold with $v$ in place of $\nabla_{\mathbb{X}} u$ for q.e. $(s, x) \in Q_{\hat{T}}$. Hence, for every $M \in \mathbb{N}$,

$\left\|\nabla_{\mathbb{X}} u_{n}-v\right\|_{M} \equiv \int_{Q_{T}}\left(E_{S, x}\left(\int_{s}^{T}\left(\left|\nabla_{\mathbb{X}} u_{n}\left(t, X_{t}\right)-v\left(t, X_{t}\right)\right|^{2} \wedge M\right) d t\right)^{1 / 2}\right) \varrho^{2}(x) d x d s \rightarrow 0$

as $n \rightarrow+\infty$. Moreover, by the definition of the space $W^{0,1}(\mathbb{X})$, for each $n \in \mathbb{N}$ there is a sequence $\left\{u_{n, k}\right\} \subset C_{c}^{\infty}\left(Q_{T}\right)$ such that

$$
\left\|\nabla u_{n, m}-\nabla_{\mathbb{X}} u_{n}\right\|_{M} \rightarrow 0
$$

as $m \rightarrow+\infty$. Put

$$
\begin{gathered}
\left\|\nabla u_{n, m}-\nabla_{\mathbb{X}} u_{n}\right\|=\sum_{M=1}^{+\infty} \frac{\left\|\nabla u_{n, m}-\nabla_{\mathbb{X}} u_{n}\right\|_{M} \wedge 1}{2^{M}}, \\
\left\|\nabla_{\mathbb{X}} u_{n}-v\right\|=\sum_{M=1}^{+\infty} \frac{\left\|\nabla_{\mathbb{X}} u_{n}-v\right\|_{M} \wedge 1}{2^{M}}
\end{gathered}
$$

By what has already been proven, there exists a subsequence $\left\{\nabla u_{n_{k}, m_{k}}\right\}$ such that $\left\|\nabla u_{n_{k}, m_{k}}-v\right\| \rightarrow 0$ as $k \rightarrow+\infty$. Therefore applying the diagonal method and [16, Proposition 3.3] we conclude that there is a further subsequence (still denoted by $\left.\left(n_{k}, m_{k}\right)\right)$ such that for q.e. $(s, x) \in Q_{\hat{T}}$,

$$
\int_{s}^{T}\left|\nabla u_{n_{k}, m_{k}}\left(t, X_{t}\right)-v\left(t, X_{t}\right)\right|^{2} \rightarrow 0 \text { in probability } P_{s, x}
$$

which when combined with Proposition 4.5 implies that $v=\nabla_{\mathbb{X}} u$.

Remark 4.7 With analogy to the notion of a generalization of Sobolev spaces introduced in [4] one can define the space $\mathcal{T}_{2, e}^{0,1}$ (which is not a linear) of measurable functions $u$ on $Q_{T}$ such that $T_{k}(u) \in \mathcal{F}$ for every $k \in \mathbb{N}$. This space is used to cope with PDEs with measure data (see [11]). It is worth mentioning that in general $W^{0,1}(\mathbb{X}) \subsetneq \mathcal{T}_{2, e}^{0,1}$ and $\mathcal{T}_{2, \varrho}^{0,1} \subsetneq W^{0,1}(\mathbb{X})$. To see this, let us put $d=1$ and consider functions $f, g: Q_{T} \rightarrow \mathbb{R}$ defined by $f(\bar{x})=\sin \frac{1}{|\bar{x}|}, g(\bar{x})=\frac{1}{|x|}$, where $\bar{x}=$ $(t, x) \in Q_{T}$. Then $f \in W^{0,1}(\mathbb{X})$ but $f \notin \mathcal{T}_{2, e}^{0,1}$ since $\operatorname{cap}_{L}(\{0,0\})=0$ and $f \in C^{\infty}\left(Q_{T} \backslash\right.$ $\{0,0\})$. On the other hand, $g \in \mathcal{T}_{2, e}^{0,1}$ but $g \notin W^{0,1}(\mathbb{X})$ because cap ${ }_{L}(\{0\} \times \mathbb{R})>0$ and $P_{0, x}\left(\int_{0}^{T}\left|g\left(t, X_{t}\right)\right|^{2} d t<\infty\right)=0$ for every $x \in \mathbb{R}$. Note, however, that in [17] it is proved that solutions to $\operatorname{PDE}(\varphi, \mu)$ with $\mu \in \mathcal{M}_{0}$ belong to the space $W^{0,1}(\mathbb{X})$. 


\section{Existence and stochastic representation of solutions of the obstacle problem}

In the sequel, $f_{u}$ stands for the function

$$
f_{u}(t, x)=f(t, x, u(t, x), \nabla u(t, x)), \quad(t, x) \in Q_{T} .
$$

Let us recall that every functional $f \in \mathcal{F}^{\prime}$ is of the form $f=f^{0}$ - divf for some $f^{0}, \mathbf{f}=\left(f^{1}, \ldots, f^{d}\right) \in \mathcal{H}$, i.e. $\langle f, \eta\rangle_{T}=\left\langle f^{0}, \eta\right\rangle_{2, \varrho, T}+\left\langle\mathbf{f}, \nabla\left(\varrho^{2} \eta\right)\right\rangle_{2, T}$.

\subsection{Obstacle Problem with One Barrier}

Definition Let $f \in \mathcal{W}_{\varrho}^{\prime}$.

(a) We say that $u \in \mathcal{F}$ is a weak solution of the Cauchy problem

$$
\frac{\partial u}{\partial t}+L_{t} u=-f, \quad u(T)=\varphi
$$

$(\operatorname{PDE}(\varphi, f)$ for short) if

$$
\left\langle u, \frac{\partial \eta}{\partial t}\right\rangle_{T}-\left\langle L_{t} u, \eta\right\rangle_{T}=(\varphi, \eta(T))+\langle f, \eta\rangle_{T}
$$

for every $\eta \in \mathcal{W}_{\varrho}$ such that $\eta(0)=0$.

(b) We say that $u \in \mathcal{W}_{\varrho}$ is a strong solution of $\operatorname{PDE}(\varphi, f)$ if

$$
\left\langle\frac{\partial u}{\partial t}, \eta\right\rangle_{T}+\left\langle L_{t} u, \eta\right\rangle_{T}=-\langle f, \eta\rangle_{T}, \quad u(T)=\varphi
$$

for every $\eta \in \mathcal{F}$.

(c) Assume that $f$ satisfies (H2). We say that $u$ is a strong solution of the semilinear Cauchy problem with terminal condition $\varphi$ and generator $f$ if (b) is satisfied with $f$ replaced by $f_{u}$.

It is known that for any $\varphi \in H, f \in \mathcal{W}_{\varrho}^{\prime}$ there exists a unique weak solution of $\operatorname{PDE}(\varphi, f)$ (see [11]). From the definition of a weak solution it follows immediately that if $f \in \mathcal{F}^{\prime}$ then there exists a strong solution of $\operatorname{PDE}(\varphi, f)$. From [20] it follows that under (H1)-(H3) there exists a unique strong solution of the semilinear Cauchy problem $\operatorname{PDE}(\varphi, f)$.

Now we recall the definition introduced in [17] of solution of Eq. 1.1 but with one barrier. The abbreviation $\underline{\mathrm{OP}}(\varphi, f, h)(\operatorname{resp} . \overline{\mathrm{OP}}(\varphi, f, h))$ refers to the problem (1.1) with $j(t, x, \cdot)=\mathbf{I}_{[h(t, x),+\infty)}(\cdot)\left(\operatorname{resp} . j(t, x, \cdot)=\mathbf{I}_{(-\infty, h(t, x)]}(\cdot)\right)$.

Definition Let (H1)-(H3) hold. We say that a pair $(u, \mu)$ is a solution of $\underline{\mathrm{OP}}(\varphi, f, h)$ (resp. $\overline{\mathrm{OP}}(\varphi, f, h))$ if $u \in \mathcal{P}_{f}\left(\right.$ resp. $\left.u \in \overline{\mathcal{P}}_{f}\right), \mu \in S_{0}$ (resp. $\left.-\mu \in S_{0}\right)$ and

(a) for every $\eta \in \mathcal{W}_{\varrho}$ such that $\eta(0) \equiv 0$,

$$
\left\langle u, \frac{\partial \eta}{\partial t}\right\rangle_{T}-\left\langle L_{t} u, \eta\right\rangle_{T}=(\varphi, \eta(T))+\left(f_{u}, \eta\right)_{T}+\int_{Q_{T}} \eta \varrho^{2} d \mu,
$$

(b) $u \geq h$ (resp. $u \leq h)$ a.e., 
(c) for q.e. $(s, x) \in Q_{\hat{T}}$,

$$
\int_{s}^{T}\left(\bar{u}_{-}\left(t, X_{t}\right)-h_{-}^{*}\left(t, X_{t}\right)\right) d \mu\left(t, X_{t}\right)=0, \quad P_{s, x} \text { a.s. }
$$

for every $h^{*} \in \mathcal{D}_{\varrho}^{2}$ such that $h \leq h^{*} \leq \bar{u}$ (resp. $h \geq h^{*} \geq \bar{u}$ ) a.e., where $\bar{u}$ is a quasicàdlàg version of $u$ (here and in what follows given a measurable function $v$ on $Q_{T}$ we denote by $v_{-}\left(t, X_{t}\right)$ the $\left.\operatorname{limit}_{s<t, s \rightarrow t} v\left(t, X_{t}\right)\right)$.

In [17] it is proved that under $(\mathrm{H} 1)-(\mathrm{H} 3),\left(\mathrm{H} 4^{*}\right)$ (in the case of one upper (resp. lower) barrier we take $h_{1} \equiv-\infty\left(\right.$ resp. $\left.h_{2} \equiv+\infty\right)$ in $\left.\left(\mathrm{H} 4^{*}\right)\right)$ there exist unique solutions of the problems $\underline{\mathrm{OP}}(\varphi, f, h), \overline{\mathrm{OP}}(\varphi, f, h)$.

5.2 Quasi-Continuous Distinct Obstacles ( $\mathbf{h}_{\mathbf{1}}<\mathbf{h}_{\mathbf{2}}$ q.e. $)$

Let $S_{c}$ denote the space of all measures $\mu \in S$ such that $d \mu(\cdot, X.) \in \mathcal{A}_{c}(s, x, T)$ for q.e. $(s, x) \in Q_{\hat{T}}$. Recall that by Corollary $4.4, S_{\varrho}^{2} \subset C([0, T] ; H)$.

Definition We say that a pair $(u, \mu)$ is a solution of $\operatorname{OP}\left(\varphi, f, h_{1}, h_{2}\right)$ if

(a) $u \in W^{0,1}(\mathbb{X}) \cap \mathcal{S}_{\varrho}^{2}, \mu^{+}, \mu^{-} \in S_{c}$, where $\mu^{+}$(resp. $-\mu^{-}$is the positive (resp. negative) part of the Jordan decomposition of $\mu$,

(b) for q.e. $(s, x) \in Q_{\hat{T}}$,

$$
\begin{aligned}
u\left(t, X_{t}\right)= & \varphi\left(X_{T}\right)+\int_{t}^{T} f_{u}\left(\theta, X_{\theta}\right) d \theta+\int_{t}^{T} d \mu^{+}\left(\theta, X_{\theta}\right)-\int_{t}^{T} d \mu^{-}\left(\theta, X_{\theta}\right) \\
& -\int_{t}^{T} \sigma \nabla_{\mathbb{X}} u\left(\theta, X_{\theta}\right) d B_{s, \theta}, \quad t \in[s, T], \quad P_{s, x} \text {-a.s. },
\end{aligned}
$$

(c) $h_{1} \leq u \leq h_{2}$ q.e.,

(d) $\int_{s}^{T}\left(u\left(t, X_{t}\right)-h_{1}\left(t, X_{t}\right)\right) d \mu^{+}\left(t, X_{t}\right)=\int_{s}^{T}\left(h_{2}\left(t, X_{t}\right)\right)-u\left(t, X_{t}\right) d \mu^{-}\left(t, X_{t}\right)=0$, $P_{s, x}$-a.s. for q.e. $(s, x) \in Q_{\hat{T}}$.

Theorem 5.1 Let assumptions (H1)-(H4) hold. Then there exists a unique solution $(u, \mu)$ of the problem $O P\left(\varphi, f, h_{1}, h_{2}\right)$.

Proof By Proposition 2.4, for q.e. $(s, x) \in Q_{\hat{T}}$ there exists a unique solution $\left(Y^{s, x}, Z^{s, x}, K^{s, x}\right)$ of $\operatorname{RBSDE}_{s, x}\left(\varphi, f, h_{1}, h_{2}\right)$. Let $\left(Y^{s, x, n}, Z^{s, x, n}, A^{s, x, n}\right)$ be a unique solution of $\left.\operatorname{RBSDE}_{s, x}\left(\varphi, f+n\left(y-h_{1}\right)\right)^{-}, h_{2}\right)$. Then by [17, Theorem 5.3] there exists a unique solution $\left(u_{n}, v_{n}\right) \in\left(\mathcal{S}_{\varrho}^{2} \cap \mathcal{F}\right) \otimes S_{0}$ of $\overline{\mathrm{OP}}\left(\varphi, f+n\left(y-h_{1}\right)^{-}, h_{2}\right)$ such that for q.e. $(s, x) \in Q_{\hat{T}}$,

$$
\left(Y^{s, x, n}, Z^{s, x, n}\right)=\left(u_{n}(\cdot, X .), \sigma \nabla u(\cdot, X .)\right) \quad \text { in } \quad \mathcal{S}^{2}(s, x, T) \otimes \mathcal{M}^{2}(s, x, T)
$$

and

$$
d A_{s, \cdot}^{s, x, n}=d v_{n}(\cdot, X .) .
$$

From [15] it follows that for q.e. $(s, x) \in Q_{\hat{T}}$,

$$
E_{s, x} \sup _{s \leq t \leq T}\left|Y_{t}^{s, x, n}-Y_{t}^{s, x}\right|^{2} \rightarrow 0,
$$


and there is a family $\left\{\gamma_{n}^{s, x}\right\}$ of stationary sequences of stopping times (i.e. such that for $P_{s, x}$-a.e. $\omega \in \Omega$ there exists $n_{0}(\omega) \in \mathbb{N}$ such that $\left.\gamma_{n_{0}(\omega)}^{s, x}=T\right)$ such that for every $k \in \mathbb{N}$,

$$
E_{s, x} \int_{s}^{\gamma_{k}^{s, x}}\left|Z_{\theta}^{s, x, n}-Z_{\theta}^{s, x}\right|^{2} d \theta \rightarrow 0
$$

and

$$
E_{s, x} \sup _{s \leq t \leq \gamma_{k}^{s, x}}\left(\left|A_{t}^{s, x, n}-K_{t}^{s, x,-}\right|^{2}+\left|K_{t}^{s, x, n}-K_{t}^{s, x,+}\right|^{2}\right) \rightarrow 0
$$

as $n \rightarrow+\infty$, where $d K^{s, x, n}=d \mu_{n}\left(\cdot, X\right.$.), $d \mu_{n}=n\left(u_{n}-h_{1}\right)^{-} d m_{T}$. From Eqs. 5.1, 5.2 we deduce that if we set $u(s, x)=\lim _{n \rightarrow+\infty} u_{n}(s, x)$ if the limit exists, and $u(s, x)=$ 0 otherwise, then $u \in \mathcal{S}_{\varrho}^{2}$ and $u\left(t, X_{t}\right)=Y_{t}^{s, x}, t \in[s, T], P_{s, x}$-a.s. for q.e. $(s, x) \in Q_{\hat{T}}$. Furthermore, from Eqs. 5.1, 5.3 it follows that for q.e. $(s, x) \in Q_{\hat{T}}$,

$$
\int_{s}^{T}\left|\nabla\left(u_{m}-u_{n}\right)\right|^{2}\left(t, X_{t}\right) d t \rightarrow 0 \text { in probability } P_{s, x}
$$

as $n, m \rightarrow \infty$. By the above and Proposition 4.5, $u \in W_{2}^{0,1}(\mathbb{X})$ and

$$
Z^{s, x}=\sigma \nabla_{\mathbb{X}} u(\cdot, X .) \quad \text { in } \quad \mathcal{M}(s, x, T)
$$

for q.e. $(s, x) \in Q_{\hat{T}}$. By Eq. 5.4, the sequence of random measures $\left\{d \mu_{n}(\cdot, X\right.$.) $\}$ satisfies the assumptions of [17, Lemma 4.16]. Therefore there exists a random measure $d C$ such that

$$
d K_{s, \cdot}^{s, x}=d C, \quad P_{s, x} \text {-a.s. }
$$

for q.e. $(s, x) \in Q_{\hat{T}}$. By Proposition 3.11, there exists a measure $\mu_{1} \in S_{c}$ such that $d \mu_{1}(\cdot, X)=.d C$. Similarly, there exists $\mu_{2} \in S_{c}$ such that $d A_{s, \cdot}^{s, x}=d \mu_{2}(\cdot, X$.), $P_{s, x}$-a.s. for q.e. $(s, x) \in Q_{\hat{T}}$. From this and the definition of a solution of $\operatorname{RBSDE}_{s, x}\left(\varphi, f, h_{1}, h_{2}\right)$ it follows that $(u, \mu)$, where $\mu=\mu_{1}-\mu_{2}$, is a solution of $\operatorname{OP}\left(\varphi, f, h_{1}, h_{2}\right)$ (it is clear that $\left.\mu_{1}=\mu^{+}, \mu_{2}=\mu^{-}\right)$. Uniqueness of the solution is a direct consequence of uniqueness for solutions of $\operatorname{RBSDE}_{s, x}\left(\varphi, f, h_{1}, h_{2}\right)$.

Remark 5.2 Let us point out that in general the integrals $E_{s, x} \int_{s}^{T} d \mu^{+}\left(\theta, X_{\theta}\right)$ and $E_{s, x} \int_{s}^{T} d \mu^{-}\left(\theta, X_{\theta}\right)$ are not finite. However, if we define $\gamma_{n}^{s, x}$ as the proof of Theorem 5.1, then for q.e. $(s, x) \in Q_{\hat{T}}, E_{s, x} \int_{s}^{\gamma_{n}^{s, x}} d \mu^{+}\left(\theta, X_{\theta}\right)+E_{s, x} \int_{s}^{\gamma_{n}^{s, x}} d \mu^{-}\left(\theta, X_{\theta}\right)<\infty$. Moreover, from condition (b) of the definition of a solution of the obstacle problem and properties of $u$ it follows that the $\operatorname{limit}_{n \rightarrow+\infty} E_{s, x} \int_{s}^{\gamma_{n}^{s, x}} d \mu\left(\theta, X_{\theta}\right)$ is well defined, and if we put

$$
E_{s, x} \int_{s}^{T} d \mu\left(\theta, X_{\theta}\right)=\lim _{n \rightarrow+\infty} E_{s, x} \int_{s}^{\gamma_{n}^{s, x}} d \mu\left(\theta, X_{\theta}\right),
$$

then

$$
u(s, x)=E_{s, x}\left(\varphi\left(X_{T}\right)+\int_{s}^{T} f_{u}\left(t, X_{t}\right) d t+\int_{s}^{T} d \mu\left(t, X_{t}\right)\right) .
$$

Using Theorem 5.1 one can generalize the well known Lewy-Stampacchia inequality (see [28]) to the case of non-Radon measures. 
Remark 5.3 Let us assume that $d V^{i}=d \delta^{i}\left(\cdot, X\right.$.), $Z^{i}=v^{i}(\cdot, X),. i=1,2$, for some $v^{i} \in$ $\mathcal{B}\left(Q_{T}\right)$ and measures $\delta^{i} \in S_{c}$. Then applying Proposition 6.1 to a unique solution of $\operatorname{RBSDE}_{s, x}\left(\varphi, f, h_{1}, h_{2}\right)$ we get by Theorem 5.1 the following stochastic version of the Lewy-Stampacchia inequality:

$$
\begin{aligned}
& d \mu^{+}\left(t, X_{t}\right) \leq \mathbf{1}_{\left\{u\left(t, X_{t}\right)=h_{1}\left(t, X_{t}\right)\right\}}\left(f\left(t, h^{1}\left(t, X_{t}\right), v^{1}\left(t, X_{t}\right)\right) d t+d \delta^{1}\left(t, X_{t}\right)\right)^{-}, \\
& d \mu^{-}\left(t, X_{t}\right) \leq \mathbf{1}_{\left\{u\left(t, X_{t}\right)=h_{2}\left(t, X_{t}\right)\right\}}\left(f\left(t, h^{2}\left(t, X_{t}\right), v^{2}\left(t, X_{t}\right)\right) d t+d \delta^{2}\left(t, X_{t}\right)\right)^{+} .
\end{aligned}
$$

From the stochastic version we get the deterministic version:

$$
\begin{aligned}
& d \mu^{+}(t, y) \leq \mathbf{1}_{\left\{u=h_{1}\right\}}\left(f\left(t, h_{1}(t, y), v^{1}(t, y)\right) d m_{T}(t, y)+d \delta^{1}(t, y)\right)^{-}, \\
& d \mu^{-}(t, y) \leq \mathbf{1}_{\left\{u=h_{2}\right\}}\left(f\left(t, h_{2}(t, y), v^{2}(t, y)\right) d m_{T}(t, y)+d \delta^{2}(t, y)\right)^{+} .
\end{aligned}
$$

The following proposition is a parabolic analogue of a result proved in the elliptic case in [25].

Proposition 5.4 Assume that (H1)-(H4) are satisfied and, in addition, $h_{1} \in \mathcal{W}_{\varrho}$, $\mathcal{L}_{f} h_{1} \in\left(\mathcal{F}^{\prime}\right)^{+}-\left(\mathcal{F}^{\prime}\right)^{+}$. Let $(u, \mu)$ be a unique solution of $O P\left(\varphi, f, h_{1}, h_{2}\right)$. Then

$$
\mu^{+} \leq \mathbf{1}_{\left\{u=h_{1}\right\}} d\left(\mathcal{L}_{f} h_{1}\right)^{-} .
$$

Proof From [16, Theorem 4.3] it follows that

$$
d h_{1}\left(t, X_{t}\right)=-f_{h}\left(t, X_{t}\right) d t+d\left(\mathcal{L}_{f} h_{1}\right)^{+}\left(t, X_{t}\right)-d\left(\mathcal{L}_{f} h_{1}\right)^{-}\left(t, X_{t}\right)+\sigma \nabla h_{1}\left(t, X_{t}\right) d B_{s, t},
$$

$P_{s, x}$-a.s., for q.e. $(s, x) \in Q_{\hat{T}}$. Therefore Eq. 5.5 follows from Remark 5.3.

As a corollary to Theorem 5.1 we get also the following important result.

Theorem 5.5 Let assumptions (H1)-(H4) hold and let $u$ be the first component of a solution of $\mathrm{OP}\left(\varphi, f, h_{1}, h_{2}\right)$. Then for q.e. $(s, x) \in Q_{\hat{T}}$,

$$
\begin{aligned}
u\left(t, X_{t}\right)= & \underset{\sigma \in \mathcal{T}_{t}^{s}}{\operatorname{ess} \inf _{\tau \in \mathcal{T}_{t}^{s}}} \operatorname{ess~up}_{s, x}\left(\int_{t}^{\tau \wedge \sigma} f_{u}\left(\theta, X_{\theta}\right) d \theta+h_{1}\left(\tau, X_{\tau}\right) \mathbf{1}_{\{\tau \leq \sigma<T\}}\right. \\
& \left.+h_{2}\left(\sigma, X_{\sigma}\right) \mathbf{1}_{\{\sigma<\tau\}}+\varphi\left(X_{T}\right) \mathbf{1}_{\{\sigma=\tau=T\}} \mid \mathcal{G}_{t}^{s}\right), \quad t \in[s, T], \quad P_{s, x} \text {-a.s., }
\end{aligned}
$$

where $\mathcal{T}_{t}^{s}=\left\{\tau \in \mathcal{T}^{s}: t \leq \tau \leq T\right\}$ and $\mathcal{T}^{s}$ denote the set of all $\left\{\mathcal{G}_{t}^{s}\right\}$-stopping times.

Proof Follows immediately form Theorem 5.1 and [15, Theorem 3.8].

The following example shows that in general the measures $\mu, v$ appearing in the definition of a solution of the problem $\mathrm{OP}\left(\varphi, f, h_{1}, h_{2}\right)$ need not be Radon measures.

Example 5.6 Let $h: \mathbb{R}^{d} \rightarrow \mathbb{R}, h(x)=\sin \frac{1}{|x|^{2}}$ for $x \neq 0$ and $h(0)=0$. One can check that

$$
\Delta h(x)=\frac{2}{|x|^{4}}\left(3 \cos \frac{1}{|x|^{2}}-2 \sin \frac{1}{|x|^{2}}\right) .
$$


For $r_{1} \geq r_{2} \geq 0$ set $B\left(r_{1}, r_{2}\right)=\left\{x \in \mathbb{R}^{d} ; r_{2} \leq|x| \leq r_{2}\right\}$. Using elementary properties of trigonometric functions one can construct a strictly decreasing sequence $\left\{\alpha_{n}\right\} \subset \mathbb{R}^{+} \cup\{+\infty\}$ such that $\bigcup_{n=1}^{+\infty} B\left(\alpha_{n+1}, \alpha_{n}\right)=\mathbb{R}^{d} \backslash\{0\}$ and $\Delta h \geq 0$ on $C^{+}=$ $\bigcup_{n=0}^{+\infty} B\left(\alpha_{2 n+2}, \alpha_{2 n+1}\right), \Delta h \leq 0$ on $C^{-}=\bigcup_{n=1}^{+\infty} B\left(\alpha_{2 n+1}, \alpha_{2 n}\right)$. Let $U \subset \mathbb{R}^{d}$ be an open set such that $0 \in U$. Then there exists $n_{0} \in \mathbb{N}$ such that

$$
\int_{U}(\Delta h)^{+}(x) d x \geq \sum_{n \geq n_{0}} \int_{B\left(\alpha_{2 n+2}, \alpha_{2 n+1}\right)} \Delta h(x) d x .
$$

On the other hand, putting $A \equiv\left\{\cos \frac{1}{|x|^{2}} \geq \sin \frac{1}{|x|^{2}}\right\}$, there is a set $B$ such that $\Delta h(x) \leq 0$ for $x \in B$ and $A=A \cap\left\{3 \cos \frac{1}{|x|^{2}} \geq 2 \sin \frac{1}{|x|^{2}}\right\} \cup B$. Therefore for $d=2$ we have

$$
\begin{aligned}
\sum_{n \geq n_{0}} \int_{B\left(\alpha_{2 n+2}, \alpha_{2 n+1}\right)} \Delta h(x) d x & \geq \sum_{n \geq n_{0}} \int_{B\left(\left(\frac{\pi}{4}+(2 n+2) \pi\right)^{-1 / 2},\left(\frac{\pi}{4}+(2 n+1) \pi\right)^{-1 / 2}\right)} \Delta h(x) d x \\
& =\sum_{n \geq n_{0}} \int_{\frac{\pi}{4}+\pi}^{\frac{\pi}{4}+2 \pi}(3 \cos t-2 \sin t) d t=\infty .
\end{aligned}
$$

Since similar arguments apply to $(\Delta h)^{-}$, we conclude that for every open set $U \subset$ $\mathbb{R}^{d}$ such that $0 \in U$,

$$
\int_{U}(\Delta h)^{+}(x) d x+\int_{U}(\Delta h)^{-}(x) d x=\infty .
$$

Let $\left\{\beta_{n}\right\},\left\{\gamma_{n}\right\} \subset \mathbb{R}^{+}$be decreasing sequences such that $\alpha_{n}>\beta_{n}>\gamma_{n}>\alpha_{n+1}$ for $n \in \mathbb{N}$. Using standard methods we construct $\tilde{h} \in C^{\infty}\left(\mathbb{R}^{d} \backslash\{0\}\right)$ such that $\tilde{h}=h$ on $B\left(\gamma_{n}, \beta_{n}\right), \tilde{h}=$ const on $B\left(\alpha_{n+1}-\frac{\left(\alpha_{n+1}-\beta_{n+1}\right)}{2}, \alpha_{n+1}+\frac{\left(\gamma_{n}-\alpha_{n+1}\right)}{2}\right)$ and $\tilde{h}$ satisfies Eq. 5.6, and then we set $D^{+(-)}=C^{+(-)} \cap\left(\bigcup_{n=0}^{+\infty} B\left(\alpha_{n+1}-\frac{\left(\alpha_{n+1}-\beta_{n+1}\right)}{2}, \alpha_{n+1}+\frac{\left(\gamma_{n}-\alpha_{n+1}\right)}{2}\right)\right)^{c}$ and

$$
\begin{gathered}
h_{1}(x)=\tilde{h}(x)\left(1-d_{D^{-}}(x) \wedge 1\right), \quad h_{2}(x)=\tilde{h}(x)\left(1-d_{D^{+}}(x) \wedge 1\right), \\
u(t, x)=\tilde{h}(x), \quad d \mu(t, x)=-(\Delta \tilde{h}) d x,
\end{gathered}
$$

where $d_{D^{-}}\left(\right.$resp. $\left.d_{D^{+}}\right)$denotes the distance function from the set $D^{-}$(resp. $\left.D^{+}\right)$. It is clear that $h_{1}<h_{2}$. We claim that the pair $(u, \mu)$ is a solution of $\operatorname{OP}\left(\tilde{h}, 0, h_{1}, h_{2}\right)$ with the operator $L_{t}=\frac{1}{2} \Delta$. Indeed, conditions (a)-(c) of the definition are clearly satisfied with $\nabla_{\mathbb{X}} u(t, x)=\nabla \tilde{h}(x)$ for $x \in \mathbb{R}^{d} \backslash\{0\}$. To see (d), let us observe that if $\Delta \tilde{h}(x)<0$ then $x \in D^{-}$, which forces $h_{1}=\tilde{h}=u$. Therefore,

$$
\int_{s}^{T}\left(u\left(t, X_{t}\right)-h_{1}\left(X_{t}\right)\right) d \mu^{+}\left(t, X_{t}\right)=\int_{s}^{T}\left(u\left(t, X_{t}\right)-h_{1}\left(X_{t}\right)\right)(\Delta \tilde{h})^{-}\left(X_{t}\right)=0
$$

$P_{s, x}$-a.s. for every $s \in[0, T), x \neq 0$. In the same manner one can show that

$$
\int_{s}^{T}\left(h_{2}\left(X_{t}\right)-u\left(t, X_{t}\right)\right) d \mu^{-}\left(t, X_{t}\right)=0
$$

$P_{s, x}$-a.s. for $s \in[0, T), x \neq 0$, which shows that $(u, \mu)$ is a solution of $\mathrm{OP}\left(\tilde{h}, 0, h_{1}, h_{2}\right)$. In view of Eq. 5.6 , the measure $\mu$ is non-Radon. 
5.3 Quasi-Continuous Distinct Obstacles Determining Radon Reaction Measure

In this section we provide additional assumptions on barriers which guarantee that the reaction measure is a Radon measure.

Set $H_{l o c}^{1}=\left\{u \in \mathbb{L}_{l o c}^{2}\left(Q_{T}\right) ; \nabla u \in \mathbb{L}_{l o c}^{2}\left(Q_{T}\right)\right\}, \quad \mathcal{F}_{l o c}=\mathbb{L}_{2}\left(0, T ; H_{l o c}^{1}\right), \quad \mathcal{W}_{l o c}=\{u \in$ $\mathcal{B}\left(Q_{T}\right) ; u \xi \in \mathcal{W}_{\varrho}$ for every $\left.\xi \in C_{c}^{\infty}\left(Q_{T}\right)\right\}$ and consider the following hypothesis.

(H5) (a) There exists $\eta \in \mathcal{W}_{\text {loc }}$ such that $h_{1} \leq \eta \leq h_{2}$ a.e.,

(b) For every $\mu \in S$ and compact subset $K \subset Q_{T}$,

$$
\begin{aligned}
& \left(\forall \eta \in \mathcal{W}_{l o c}, h_{1} \leq \eta \leq h_{2}, \int_{K}\left(\eta-h_{1}\right) d \mu<+\infty\right) \Rightarrow \mu(K)<\infty, \\
& \left(\forall \eta \in \mathcal{W}_{l o c}, h_{1} \leq \eta \leq h_{2}, \int_{K}\left(h_{2}-\eta\right) d \mu<+\infty\right) \Rightarrow \mu(K)<\infty .
\end{aligned}
$$

Let us remark that (H5) is satisfied if $h_{1}, h_{2}$ are continuous.

Theorem 5.7 Let assumptions (H1)-(H5) hold and let $(u, \mu)$ be a unique solution of $O P\left(\varphi, f, h_{1}, h_{2}\right)$. Then $u \in \mathcal{F}_{l o c}, \mu^{+}, \mu^{-} \in \mathcal{M}_{0}$.

Proof Let us define $u_{n}, \mu_{n}, v_{n}$ as in the proof Theorem 5.1, and let $\eta \in \mathcal{W}_{\text {loc }}$ be such that $h_{1} \leq \eta \leq h_{2}$ a.e.. Let $U \subset \mathbb{R}^{d}$ be an open bonded set, $U_{T}=[0, T] \times U$, and let $K$ be a compact subset of $U$. Furthermore, let $\xi \in C_{c}^{\infty}\left(U_{T}\right)$ be such that $\xi_{\mid K} \equiv 1$ and $\xi$ does not depend on $t$. Let us denote by $u_{n, m}$ a unique solution of $\operatorname{PDE}\left(\varphi, f_{n, m}\right)$ with $\left.f_{n, m}(t, x, y, z)=f(t, x, y, z)+n\left(y-h_{1}(t, x)\right)^{-}-m\left(y-h_{2}(t, x)\right)^{+}\right)$and let $d v_{n, m}=$ $m\left(u_{n, m}-h_{2}\right)^{+} d m_{T}, d \mu_{n, m}=n\left(u_{n, m}-h_{1}\right)^{-} d m_{T}$. Taking $\left(u_{n, m}-\eta\right) \xi^{2} \varrho^{-2}$ as a test function in the variational equality for $\operatorname{PDE}\left(\varphi, f_{n, m}\right)$ we get

$$
\begin{aligned}
\| u_{n, m}(t) & \xi \|_{2}^{2}+\left(a \nabla u_{n, m}, \nabla u_{n, m} \xi^{2}\right)_{2, t, T} \\
= & \|\varphi \xi\|_{2}^{2}+\left(a \nabla u_{n, m}, \nabla\left(\eta \xi^{2}\right)\right)_{2, t, T}+\left.\left(u_{n, m}(\cdot), \eta \xi^{2}\right)_{2}\right|_{t} ^{T}-2\left(a \nabla u_{n, m}, u_{n, m} \xi \nabla \xi\right)_{2, t, T} \\
& +\left(b \nabla u_{n, m},\left(u_{n, m}-\eta\right) \xi^{2}\right)_{2, t, T}+\left\langle\eta_{t}, u_{n, m} \xi^{2}\right\rangle_{t, T}+2\left(f_{u_{n, m}},\left(u_{n, m}-\eta\right) \xi^{2}\right)_{2, t, T} \\
& +2 \int_{Q_{t T}}\left(u_{n, m}-\eta\right) \xi^{2} d \mu_{n, m}-2 \int_{Q_{t T}}\left(u_{n, m}-\eta\right) \xi^{2} d v_{n, m}
\end{aligned}
$$

where $(\cdot, \cdot)_{2, t, T}$ stands for the usual inner product in $\mathbb{L}_{2}\left(Q_{t T}\right)$. From [17, Theorem 5.3] it follows that if $m \rightarrow \infty$ then $u_{n, m} \rightarrow u_{n}$ in $\mathcal{F}, v_{n, m} \rightarrow v_{n}$ in the weak* topology on $\mathcal{W}_{\varrho}^{\prime}$ and $v_{n, m}\left(u_{n, m}\right) \rightarrow v_{n}\left(u_{n}\right)$. Therefore letting $m \rightarrow \infty$ in Eq. 5.7 we get

$$
\begin{aligned}
\| u_{n}(t) & \xi \|_{2}^{2}+\left(a \nabla u_{n}, \nabla u_{n} \xi^{2}\right)_{2, t, T} \\
= & \|\varphi \xi\|_{2}^{2}+\left(a \nabla u_{n}, \nabla\left(\eta \xi^{2}\right)\right)_{2, t, T}+\left.\left(u_{n}(\cdot), \eta \xi^{2}\right)_{2}\right|_{t} ^{T}-2\left(a \nabla u_{n}, u_{n} \xi \nabla \xi\right)_{2, t, T} \\
& +\left(b \nabla u_{n},\left(u_{n}-\eta\right) \xi^{2}\right)_{2, t, T}+\left\langle\eta_{t}, u_{n} \xi^{2}\right\rangle_{t, T}+2\left(f_{u_{n}},\left(u_{n}-\eta\right) \xi^{2}\right)_{2, t, T} \\
& +2 \int_{Q_{t}}\left(u_{n}-\eta\right) \xi^{2} d \mu_{n}-2 \int_{Q_{t T}}\left(u_{n}-\eta\right) \xi^{2} d v_{n} .
\end{aligned}
$$


Observe that

$$
-\int_{Q_{t T}}\left(u_{n}-\eta\right) \xi^{2} d \mu_{n} \geq-\int_{Q_{t T}} \xi^{2}\left(u_{n}-h_{1}\right) d \mu_{n} \geq 0
$$

and

$$
\int_{Q_{t T}}\left(u_{n}-\eta\right) \xi^{2} d v_{n} \geq \int_{Q_{t T}} \xi^{2}\left(u_{n}-h_{2}\right) d v_{n}=0 .
$$

Hence

$$
\begin{aligned}
\left\|u_{n}(t) \xi\right\|_{2}^{2}+\lambda\left\|\nabla u_{n} \xi\right\|_{2, t, T}^{2} \leq & \|\varphi \xi\|_{2}^{2}+\left|\left(a \nabla u_{n}, \nabla\left(\eta \xi^{2}\right)\right)_{2, t, T}\right|+2\left|\left(a \nabla u_{n}, u_{n} \xi \nabla \xi\right)_{2, t, T}\right| \\
& +\left|\left(b \nabla u_{n},\left(u_{n}-\eta\right) \xi^{2}\right)\right|+\left|\left\langle\eta_{t}, u_{n} \xi^{2}\right\rangle_{t, T}\right| \\
& +\left|\left(u_{n}(\cdot), \eta \xi^{2}\right)_{2}\right|_{t}^{T}|+|\left(f_{u_{n}},\left(u_{n}-\eta\right) \xi^{2}\right)_{2, t, T} \mid .
\end{aligned}
$$

Using Young's inequality and Gronwall's lemma we conclude from the above that

$$
\begin{aligned}
& \left\|u_{n}(t) \xi\right\|_{2}^{2}+\left\|\nabla u_{n} \xi\right\|_{2, T}^{2} \leq C\left(\|\varphi \xi\|_{2}^{2}+\|\nabla \xi \eta\|_{2, T}^{2}+\|\xi \nabla \eta\|_{2, T}^{2}\right. \\
& \left.\quad+\|\eta \xi\|_{2, T}^{2}+\left\|u_{n} \nabla \xi\right\|_{2, T}^{2}+\left\|\eta_{t} \xi\right\|_{L_{2}\left(0, T, H^{-1}\right)}+\sup _{0 \leq t \leq T}\|\xi \eta(t)\|_{2}^{2}+\|g \xi\|_{2, T}^{2}\right) .
\end{aligned}
$$

Accordingly, $\left\{u_{n} \xi\right\}$ is bounded in $\mathcal{F}$, and hence $u \in \mathcal{F}_{\text {loc }}$ since we know that $u_{n} \rightarrow u$ pointwise. From Eqs. 5.7, 5.8 it follows that for any $\xi \in C_{c}^{\infty}\left(Q_{T}\right)$ and $\eta \in \mathcal{W}_{l o c}$ such that $h_{1} \leq \eta \leq h_{2}$

$$
\sup _{n \geq 1} \int_{Q_{T}}\left(\eta-u_{n}\right) \xi^{2} d \mu_{n}<\infty .
$$

By Eqs. 5.2, 5.4, for q.e. $(s, x) \in Q_{\hat{T}}$,

$$
\int_{s}^{T}\left(\left(\eta-u_{n}\right) \xi^{2}\right)\left(t, X_{t}\right) d \mu_{n}\left(t, X_{t}\right) \rightarrow \int_{s}^{T}\left((\eta-u) \xi^{2}\right)\left(t, X_{t}\right) d \mu^{+}\left(t, X_{t}\right)
$$

in probability $P_{s, x}$. Let $\left(X, Q_{s, x}\right)$ be a Markov process associated with the operator $A_{t}$ (see Remark 2.1). From the above convergence and Eq. 5.9 we get

$$
\begin{aligned}
\int_{Q_{T}}(\eta-u) \xi^{2} d \mu^{+} & =\int_{\mathbb{R}^{d}}\left(E_{Q_{0, x}} \int_{0}^{T}\left((\eta-u) \xi^{2}\right)\left(t, X_{t}\right) d \mu^{+}\left(t, X_{t}\right)\right) d x \\
& \leq \liminf _{n \rightarrow \infty} \int_{\mathbb{R}^{d}}\left(E_{Q_{0, x}} \int_{0}^{T}\left(\left(\eta-u_{n}\right) \xi^{2}\right)\left(t, X_{t}\right) d \mu_{n}\left(t, X_{t}\right)\right) d x \\
& =\liminf _{n \rightarrow \infty} \int_{Q_{T}}\left(\eta-u_{n}\right) \xi^{2} d \mu_{n}<\infty .
\end{aligned}
$$

On the other hand, by the definition of a solution of the obstacle problem,

$$
\int_{Q_{t T}}(\eta-u) \xi^{2} d \mu^{+}=\int_{Q_{t T}}\left(\eta-h_{1}\right) \xi^{2} d \mu^{+} .
$$

By the above and (H4), $\mu^{+} \in \mathcal{M}_{0}$. In the same manner we can see that $\mu^{-} \in \mathcal{M}_{0}$. 
Remark 5.8 If we know that a solution $(u, \mu)$ of $\mathrm{OP}\left(\varphi, f, h_{1}, h_{2}\right)$ has the same properties as the solution of Theorem 5.7, then condition (b) of the definition of a solution of the obstacle problem is equivalent to the following condition: for every $\eta \in C_{c}^{\infty}\left(Q_{T}\right)$ such that $\eta(0)=0$,

$$
\left\langle u, \frac{\partial \eta}{\partial t}\right\rangle_{T}-\left\langle L_{t} u, \eta\right\rangle_{T}=(\varphi, \eta(T))+\left(f_{u}, \eta\right)_{T}+\int_{Q_{T}} \eta \varrho^{2} d \mu .
$$

The proof of this fact is analogous to the proof of Proposition 5.10.

\subsection{Square-Integrable Obstacles Satisfying the Mokobodzki Condition}

In this section we first introduce the definition of the obstacle problem with integrable barriers via stochastic equations. Our definition generalizes the corresponding definition for one barrier introduced in [17]. Next, we prove existence and uniqueness of solutions of the problem.

We write $\mu \in \mathcal{A}_{c}^{2}\left(\mathcal{A}^{2}\right)$ if $\mu \in S$ and $\int_{s} d \mu\left(\theta, X_{\theta}\right) \in \mathcal{A}_{c}^{2}(s, x, T)\left(\mathcal{A}^{2}(s, x, T)\right)$ for q.e. $(s, x) \in Q_{\hat{T}}$. We write $\mu \in \mathcal{A}_{c, \varrho}^{2}\left(\mathcal{A}_{\varrho}^{2}\right)$ if $\mu \in \mathcal{A}_{c}^{2}\left(\mathcal{A}^{2}\right)$ and

$$
\sup _{s \in[0, T)} \int_{\mathbb{R}^{d}}\left(E_{s, x}\left(\int_{s}^{T} d \mu\left(t, X_{t}\right)\right)^{2}\right) \varrho^{2}(x) d x<\infty .
$$

Proposition $5.9 \mathcal{A}_{\varrho}^{2}=S_{0}$.

Proof Let $\mu \in \mathcal{A}_{\varrho}^{2}$. Put $\mu(\eta)=\int_{Q_{T}} \eta \varrho^{2} d \mu$ for $\eta \in \mathcal{W}_{\varrho}$. By Corollary 4.4 and Proposition 2.2,

$$
\begin{aligned}
\|\mu\|_{\mathcal{W}_{\varrho}^{\prime}} \leq \sup _{\|\eta\|_{\mathcal{S}_{Q}^{2} \leq 1}} \mu(\eta) & \leq C \sup _{\|\eta\|_{\mathcal{S}_{\varrho}^{2}} \leq 1} \int_{\mathbb{R}^{d}}\left(E_{0, x} \int_{0}^{T} \eta\left(t, X_{t}\right) d \mu\left(t, X_{t}\right)\right) \varrho^{2}(x) d x \\
& \leq C \sup _{s \in[0, T)}\left(\int_{\mathbb{R}^{d}}\left(E_{S, x} \int_{s}^{T} d \mu\left(t, X_{t}\right)\right)^{2} \varrho^{2}(x) d x\right)^{1 / 2} .
\end{aligned}
$$

Accordingly, $\mathcal{A}_{\varrho}^{2} \subset S_{0}$. The opposite inclusion follows from [17, Theorem 5.3].

Let us recall (see Section 4) that $\mathcal{P} \subset \mathcal{D}_{\varrho}^{2} \subset D([0, T] ; H)$.

Definition We say that a pair $(u, \mu)$ is a solution of $\operatorname{OP}\left(\varphi, f, h_{1}, h_{2}\right)$ if

(a) $u \in \mathcal{P}-\mathcal{P}, \mu^{+}, \mu^{-} \in \mathcal{A}_{\varrho}^{2}$ and

$$
\begin{aligned}
\bar{u}\left(t, X_{t}\right)= & \varphi\left(X_{T}\right)+\int_{t}^{T} f_{\bar{u}}\left(\theta, X_{\theta}\right) d \theta+\int_{t}^{T} d \mu^{+}\left(\theta, X_{\theta}\right)-\int_{t}^{T} d \mu^{-}\left(\theta, X_{\theta}\right) \\
& -\int_{t}^{T} \sigma \nabla \bar{u}\left(\theta, X_{\theta}\right) d B_{s, \theta}, \quad t \in[s, T], \quad P_{s, x} \text {-a.s. },
\end{aligned}
$$

(b) $h_{1} \leq u \leq h_{2}$ a.e., 
(c) for q.e. $(s, x) \in Q_{\hat{T}}, P_{s, x}$-a.s.,

$$
\begin{aligned}
\int_{s}^{T}\left(\bar{u}_{-}\left(t, X_{t}\right)-h_{1-}^{*}\left(t, X_{t}\right)\right) d \mu^{+}\left(t, X_{t}\right) & =\int_{s}^{T}\left(h_{2-}^{*}\left(t, X_{t}\right)-\bar{u}_{-}\left(t, X_{t}\right)\right) d \mu^{-}\left(t, X_{t}\right) \\
& =0
\end{aligned}
$$

for every $h_{1}^{*}, h_{2}^{*} \in \mathcal{D}_{\varrho}^{2}$ such that $h_{1} \leq h_{1}^{*} \leq \bar{u} \leq h_{2}^{*} \leq h_{2}$ a.e., where $\bar{u}$ is a quasicàdlàg version of $u$.

Proposition 5.10 If $(\mathrm{H} 1),(\mathrm{H} 2)$ are satisfied then condition (a) of the above definition is equivalent to the following condition

(a*) $u \in \mathcal{P}-\mathcal{P}, \mu^{+}, \mu^{-} \in S_{0}$ and $u$ is a unique solution of $\operatorname{PDE}(\varphi, f+\mu)$.

Proof Assume (a). Then $\mu^{+}, \mu^{-} \in S_{0}$ by Proposition 5.9. Let $\eta \in R_{\alpha}\left(C_{c}^{\infty}\left(Q_{T}\right)\right) \subset$ $D(\mathcal{L}) \subset \mathcal{W}_{\varrho}$, where $R_{\alpha}$ is the resolvent of the operator $(\mathcal{L}, D(\mathcal{L})), D(\mathcal{L})=\{u \in$ $\mathcal{F} ; \mathcal{L} u \in \mathcal{H}, u(T)=0\}$. By [17, Proposition 3.6], for q.e. $(s, x) \in Q_{\hat{T}}$,

$$
\eta\left(t, X_{t}\right)=-\int_{t}^{T} \mathcal{L} \eta\left(\theta, X_{\theta}\right) d \theta-\int_{t}^{T} \sigma \nabla \eta\left(\theta, X_{\theta}\right) d B_{s, \theta}, \quad t \in[s, T], \quad P_{s, x} \text {-a.s.. }
$$

By the integration by parts formula, for $t \in[s, T]$ we have

$$
\begin{aligned}
\eta \bar{u}\left(t, X_{t}\right)= & \int_{t}^{T} \eta\left(\theta, X_{\theta}\right) f_{u}\left(\theta, X_{\theta}\right) d \theta+\int_{t}^{T} \eta\left(\theta, X_{\theta}\right) d \mu^{+}\left(\theta, X_{\theta}\right) \\
& -\int_{t}^{T} \eta\left(\theta, X_{\theta}\right) d \mu^{-}\left(\theta, X_{\theta}\right)-\int_{t}^{T} \sigma \eta \nabla u\left(\theta, X_{\theta}\right) d B_{s, \theta}-\int_{t}^{T} u \mathcal{L} \eta\left(\theta, X_{\theta}\right) d \theta \\
& -\int_{t}^{T} u\left(\theta, X_{\theta}\right) \sigma \nabla \eta\left(\theta, X_{\theta}\right) d B_{s, \theta}-\int_{t}^{T} \sigma \nabla u\left(\theta, X_{\theta}\right) \sigma \nabla \eta\left(\theta, X_{\theta}\right) d \theta .
\end{aligned}
$$

Taking expectation with respect to the measure $Q_{s, x}$ associated with the operator $A_{t}$ we get

$$
\begin{aligned}
\langle\eta(t), \bar{u}(t)\rangle_{2}= & \left\langle f_{u}, \eta\right\rangle_{2, t, T}+\int_{Q_{t T}} \eta d \mu+\langle b \nabla u, \eta\rangle_{2, t, T} \\
& -\langle u, \mathcal{L} \eta\rangle_{2, t, T}+\langle b \nabla \eta, u\rangle_{2, t, T}-\langle a \nabla u, \nabla \eta\rangle_{2, t, T} .
\end{aligned}
$$

Let us observe now that $\left(R_{\alpha} \xi\right) \varrho^{-2} \in \mathbb{L}_{2}\left(Q_{T}\right)$ if $\xi \in C_{c}^{\infty}\left(Q_{T}\right)$. Indeed, this follows from Proposition 2.2 and the fact that

$$
R_{\alpha} \xi(s, x)=E_{s, x} \int_{0}^{T} \mathbf{1}_{[0, T]}(s+t) e^{-\alpha t} \xi\left(s+t, X_{s+t}\right) d t
$$

(see, e.g., [26]). Therefore, using strong continuity of the resolvent $R_{\alpha}$ (see [41, Proposition 2.7]) and standard approximation arguments (so far we have considered $\eta$ such that $\eta(T) \equiv 0$ ) one can prove that Eq. 5.10 holds for all $\eta \in \mathcal{W}_{\varrho}$, which shows that $u$ is a solution of $\operatorname{PDE}(\varphi, f+\mu)$. Thus, (a*) is satisfied. 
Now, let us assume that ( $\left.\mathrm{a}^{*}\right)$ is satisfied. Let $u_{1}, u_{2}$ denote solutions of problems $\operatorname{PDE}\left(\varphi, f_{u}+\mu^{+}\right)$and $\operatorname{PDE}\left(0,-\mu^{-}\right)$, respectively. By uniqueness, $u=u_{1}+u_{2}$, and by [17, Theorem 6.2], for q.e. $(s, x) \in Q_{\hat{T}}$ we have

$$
\begin{aligned}
\bar{u}_{1}\left(t, X_{t}\right)= & \varphi\left(X_{T}\right)+\int_{t}^{T} f_{u}\left(\theta, X_{\theta}\right) d \theta \\
& +\int_{t}^{T} d \mu^{+}\left(\theta, X_{\theta}\right)-\int_{t}^{T} \sigma \nabla u_{1}\left(\theta, X_{\theta}\right) d B_{s, \theta}, \quad t \in[s, T], \quad P_{s, x} \text {-a.s., }
\end{aligned}
$$

and

$$
\bar{u}_{2}\left(t, X_{t}\right)=-\int_{t}^{T} d \mu^{-}\left(\theta, X_{\theta}\right)-\int_{t}^{T} \sigma \nabla u_{2}\left(\theta, X_{\theta}\right) d B_{s, \theta}, \quad t \in[s, T], \quad P_{s, x} \text {-a.s. },
$$

which yields (a).

Remark 5.11 Let $(u, \mu)$ be a solution of $\operatorname{OP}\left(\varphi, f, h_{1}, h_{2}\right)$. Observe that from the definition of a solution of the obstacle problem it follows directly that for q.e. $(s, x) \in Q_{\hat{T}}$,

$$
\int_{s}^{T}\left(\bar{u}_{-}\left(t, X_{t}\right)-h_{-}^{*}\left(t, X_{t}\right)\right) d \mu\left(t, X_{t}\right) \leq 0, \quad P_{s, x} \text {-a.s. }
$$

for any $h^{*} \in \mathcal{D}_{\varrho}^{2}$ such that $h_{1} \leq h^{*} \leq h_{2}$ a.e.. Indeed, let $h_{1}^{*}, h_{2}^{*}$ be as in condition (c). Then

$$
\begin{gathered}
\int_{s}^{T}\left(\bar{u}_{-}\left(t, X_{t}\right)-h_{-}^{*}\left(t, X_{t}\right)\right) d \mu\left(t, X_{t}\right) \leq \int_{s}^{T}\left(\bar{u}_{-}\left(t, X_{t}\right)-\left(h^{*} \wedge h_{1}^{*}\right)_{-}\left(t, X_{t}\right)\right) d \mu^{+}\left(t, X_{t}\right) \\
-\int_{s}^{T}\left(\bar{u}_{-}\left(t, X_{t}\right)-\left(h^{*} \vee h_{2}^{*}\right)_{-}\left(t, X_{t}\right)\right) d \mu^{-}\left(t, X_{t}\right)=0 .
\end{gathered}
$$

Let us remark that it would be possible to give definition of the obstacle problem with condition (c) replaced by Eq. 5.11, but adopting such a definition we would have less information on reaction measures, because we would not know a priori that there exists a decomposition of the measure $\mu$ such that (c) is satisfied.

Proposition 5.12 Let assumptions ( $\mathrm{H} 1)-(\mathrm{H} 3)$ hold. Then there exists at most one solution of $\mathrm{OP}\left(\varphi, f, h_{1}, h_{2}\right)$.

Proof Let $\left(u_{1}, \mu_{1}\right),\left(u_{2}, \mu_{2}\right)$ be two solutions of $\mathrm{OP}\left(\varphi, f, h_{1}, h_{2}\right)$. Write $u=u_{1}-u_{2}$, $F=f_{u_{1}}-f_{u_{2}}, \mu=\mu_{1}-\mu_{2}$. Then for q.e. $(s, x) \in Q_{\hat{T}}$ we have

$$
\bar{u}\left(t, X_{t}\right)=\int_{t}^{T} F\left(\theta, X_{\theta}\right) d \theta+\int_{t}^{T} d \mu\left(\theta, X_{\theta}\right)-\int_{t}^{T} \sigma \nabla u\left(\theta, X_{\theta}\right) d B_{s, \theta}, \quad t \in[s, T]
$$

$P_{s, x}$-a.s.. By Itô's formula,

$$
\begin{gathered}
E_{s, x}\left|\bar{u}\left(t, X_{t}\right)\right|^{2}+E_{s, x} \int_{t}^{T}\left|\sigma \nabla \bar{u}\left(\theta, X_{\theta}\right)\right|^{2} d \theta+E_{s, x} \sum_{t<\theta \leq T}\left|\Delta \int_{s} d \mu\left(\theta, X_{\theta}\right)\right|^{2} \\
\quad=2 E_{s, x} \int_{t}^{T} F\left(\theta, X_{\theta}\right) \bar{u}\left(\theta, X_{\theta}\right) d \theta+2 E_{s, x} \int_{t}^{T} \bar{u}_{-}\left(\theta, X_{\theta}\right) d \mu\left(\theta, X_{\theta}\right)
\end{gathered}
$$


for $t \in[s, T]$. By Remark 5.11, for q.e. $(s, x) \in Q_{\hat{T}}$,

$$
\begin{aligned}
\int_{t}^{T} \bar{u}_{-}\left(\theta, X_{\theta}\right) d \mu\left(\theta, X_{\theta}\right)= & \int_{t}^{T}\left(\bar{u}_{1-}-\bar{u}_{2-}\right)\left(\theta, X_{\theta}\right) d \mu_{1}\left(\theta, X_{\theta}\right) \\
& +\int_{t}^{T}\left(\bar{u}_{1-}-\bar{u}_{2-}\right)\left(\theta, X_{\theta}\right) d \mu_{2}\left(\theta, X_{\theta}\right) \leq 0, \quad t \in[s, T]
\end{aligned}
$$

$P_{s, x}$-a.s., which implies that for $t \in[s, T]$,

$$
E_{s, x}\left|\bar{u}\left(t, X_{t}\right)\right|^{2}+E_{s, x} \int_{t}^{T}\left|\sigma \nabla \bar{u}\left(\theta, X_{\theta}\right)\right|^{2} d \theta \leq 2 E_{s, x} \int_{t}^{T} F\left(\theta, X_{\theta}\right) \bar{u}\left(\theta, X_{\theta}\right) d \theta .
$$

Using Young's inequality and then Gronwall's lemma we deduce from the above that $E_{s, x}\left|\bar{u}\left(t, X_{t}\right)\right|^{2}=0$ for q.e. $(s, x) \in Q_{\hat{T}}$, which when combined with Proposition 2.2 and [17, Proposition 3.4] shows that $\bar{u}_{1}=\bar{u}_{2}$ q.e.. Hence, by condition (a*) of the definition of a solution of the obstacle problem, $\int_{Q_{T}} \eta d \mu_{1}=\int_{Q_{T}} \eta d \mu_{2}$ for every $\eta \in C_{c}^{\infty}\left(Q_{T}\right)$ such that $\eta(0) \equiv 0$. Accordingly, $\mu_{1}$ coincides with $\mu_{2}$ on $(0, T] \times \mathbb{R}^{d}$. Since $\mu_{1}(0)=\mu_{2}(0)=0$, this completes the proof.

Theorem 5.13 Let assumptions (H1)-(H3), (H4*) hold.

(i) There exists a unique solution $(u, \mu)$ of $O P\left(\varphi, f, h_{1}, h_{2}\right)$.

(ii) Let $\bar{u}_{n}$ be a quasi-continuous version of a solution $u_{n}$ of the problem

$$
\frac{\partial u_{n}}{\partial t}+L_{t} u_{n}=-f_{u_{n}}-n\left(u_{n}-h_{1}\right)^{-}+n\left(u_{n}-h_{2}\right)^{+}, \quad u_{n}(T)=\varphi .
$$

Then $\bar{u}_{n} \rightarrow \bar{u}$ q.e. and in $\mathcal{H}, \nabla u_{n} \rightarrow \nabla u$ in $\mathbb{L}_{p, \varrho}\left(Q_{T}\right)$ for $p \in[1,2)$, and if $h_{1}, h_{2}$ are quasi-continuous then the last convergence holds true for $p=2$, too.

(iii) Let

$$
\begin{aligned}
F= & \left\{(s, x) \in Q_{\hat{T}} ; E_{s, x}\left(\int_{s}^{T} d \mathcal{E}\left(\phi^{+}\right)\left(t, X_{t}\right)\right)^{2}+E_{s, x}\left(\int_{s}^{T} d \mathcal{E}\left(\phi^{-}\right)\left(t, X_{t}\right)\right)^{2}\right. \\
& +E_{s, x} \int_{s}^{T}\left|g\left(t, X_{t}\right)\right|^{2} d t+E_{s, x} \underset{s \leq t \leq T}{\operatorname{ess} \sup _{1}\left|h_{1}^{+}\left(t, X_{t}\right)\right|^{2}} \\
& \left.+E_{s, x} \underset{s \leq t \leq T}{\operatorname{ess} \sup }\left|h_{2}^{-}\left(t, X_{t}\right)\right|^{2}<\infty\right\} .
\end{aligned}
$$

Then $\operatorname{cap}_{\mathcal{L}}\left(F^{c}\right)=0$ and for every $(s, x) \in F$ the triple

$$
\left(\bar{u}(\cdot, X .), \sigma \nabla u(\cdot, X), \int_{s}^{\cdot} d \mu\left(t, X_{t}\right)\right)
$$

is a unique solution of $\operatorname{RBSDE}_{s, x}\left(\varphi, f, h_{1}, h_{2}\right)$.

Proof The fact that $\operatorname{cap}_{\mathcal{L}}\left(F^{c}\right)=0$ follows from Proposition 2.5. By [17, Proposition 3.6], for every $(s, x) \in F$ there is a unique solution $\left(Y^{s, x, n}, Z^{s, x, n}\right)$ of $\operatorname{BSDE}_{s, x}\left(\varphi, f_{n}\right)$ with $\left.f_{n}(t, x, y, z)=f(t, x, y, z)+n\left(y-h_{1}(t, x)\right)^{-}-n\left(y-h_{2}(t, x)\right)^{+}\right)$and

$$
\left(Y^{s, x, n}, Z^{s, x, n}\right)=\left(\bar{u}_{n}\left(t, X_{t}\right), \sigma \nabla u_{n}\left(t, X_{t}\right)\right) \text { in } \mathcal{S}^{2}(s, x, T) \otimes \mathcal{M}^{2}(s, x, T),
$$


where $\bar{u}_{n}$ is a quasi-continuous version of a unique solution $u_{n}$ of $\operatorname{PDE}\left(\varphi, f_{n}\right)$. Let $(s, x) \in F$. Then by Theorem 6.3 there is a unique solution $\left(Y^{s, x}, Z^{s, x}, K^{s, x}\right)$ of $\operatorname{RBSDE}_{s, x}\left(\varphi, f, h_{1}, h_{2}\right)$. From Theorem 6.3(ii) we deduce that the function $\bar{u}$ defined by $\bar{u}(s, x)=\lim _{n \rightarrow+\infty} \bar{u}_{n}(s, x)$ if the limit exists, and $\bar{u}(s, x)=0$ otherwise, is quasi-càdlàg and $\bar{u}\left(\cdot, X\right.$.) $=Y^{s, x}$ in $\mathcal{D}^{2}(s, x, T)$. Moreover, by Theorem 6.3(ii) and Proposition 2.2,

$$
\lim _{n, m \rightarrow+\infty}\left(\left\|u_{n}-u_{m}\right\|_{\mathcal{H}}+\left\|\nabla u_{n}-\nabla u_{n}\right\|_{p, \varrho, T}\right)=0
$$

for $p \in[1,2$ ), while by Theorem 6.3(i) and Proposition 2.2, there is $C>0$ such that

$$
\sup _{t \in[0, T]}\left\|\bar{u}_{n}(t)\right\|_{H}+\left\|\nabla u_{n}\right\|_{\mathcal{H}}<C .
$$

Hence $u \in \mathcal{F}, u_{n} \rightarrow u_{n}$ in $\mathcal{H}$ and $\nabla u_{n} \rightarrow \nabla u$ in $\mathbb{L}_{p, \varrho}\left(Q_{T}\right)$ for $p \in[1,2)$. If $h_{1}, h_{2}$ are quasi-continuous, then by Theorem 6.3(ii) and Proposition 2.2, $\nabla u_{n} \rightarrow \nabla u$ in $\mathcal{H}$. Let us fix $\delta>0$. Then

$$
\begin{gathered}
E_{s, x} \int_{s+\delta}^{T}\left|Z_{t}^{s, x}-\sigma \nabla u\left(t, X_{t}\right)\right|^{p} d t=\lim _{n \rightarrow+\infty} E_{s, x} \int_{s+\delta}^{T}\left|\sigma\left(\nabla u_{n}-\nabla u\right)\left(t, X_{t}\right)\right|^{p} d t \\
\quad=\lim _{n \rightarrow+\infty} \int_{Q_{s+\delta, T}}\left|\sigma\left(\nabla u_{n}-\nabla u\right)\right|^{p} p(s, x, t, y) d t d y=0,
\end{gathered}
$$

the last equality being a consequence of the fact that $p(s, x, \cdot, \cdot)$ is bounded on $Q_{s+\delta, T}$. Hence $\sigma \nabla u(\cdot, X$. $)=Z^{s, x}$ in $\mathcal{M}^{2}(s, x, T)$ for $(s, x) \in F$.

Now, let us define the quadruple $\left(\underline{Y}^{s, x, n}, \underline{Z}^{s, x, n}, \underline{K}^{s, x, n}, \underline{A}^{s, x, n}\right)$ as in Theorem 6.3 (iii) but on the filtered probability space $\left(\Omega, \mathcal{G},\left\{\mathcal{G}_{t}^{s}\right\}, P_{s, x}\right)$. Then by [17, Theorem 5.3],

$$
\begin{gathered}
\left(\underline{Y}^{s, x, n}, \underline{Z}^{s, x, n}\right)=\left(\bar{v}_{n}(\cdot, X .), \sigma \nabla v_{n}(\cdot . X .)\right) \text { in } \mathcal{D}^{2}(s, x, T) \otimes \mathcal{M}^{2}(s, x, T), \\
d \underline{K}^{s, x, n}=d \delta_{n}^{1}(\cdot, X .)
\end{gathered}
$$

for $(s, x) \in F$, where $\left(v_{n}, \delta_{n}^{1}\right)$ is a unique solution of $\underline{\mathrm{OP}}\left(\varphi, \tilde{f}_{n}, h_{1}\right)$ with $\tilde{f}_{n}(t, x, y, z)=$ $f(t, x, y, z)-n\left(y-h_{2}(t, x)\right)^{+}$. Write $\delta_{n}^{2}=n\left(v_{n}-h_{2}\right)^{+} d m_{T}$. Then

$$
d \underline{A}^{s, x, n}=d \delta_{n}^{2}(\cdot, X .) .
$$

Therefore, by Theorem 6.3(iii) and [17, Lemma 4.16], there exists a random measure $d A$ such that for every $(s, x) \in F$,

$$
d A=d K^{s, x,-}, \quad P_{s, x}-\text { a.s.. }
$$

Let us put

$$
\begin{aligned}
C_{t}(\omega)= & \bar{u}\left(Z_{t}(\omega)\right)-\bar{u}\left(Z_{\tau(\omega)}(\omega)\right)-\int_{\tau(\omega)}^{\tau(\omega) \wedge t} f_{u}\left(\theta, X_{\theta}(\omega)\right) d \theta \\
& +\int_{0}^{t} d A_{\theta}(\omega)+\left(\int_{\tau}^{\tau \wedge t} \sigma \nabla u\left(\theta, X_{\theta}\right) d B_{\tau, \theta}\right)(\omega)
\end{aligned}
$$

for $\omega \in \Omega$ such that $C$. $(\omega)$ is a càdlàg nondecreasing function, and we put $C .(\omega) \equiv 0$ otherwise. Of course, $d C$ is a random measure and

$$
d C=d K^{s, x,+}, \quad P_{s, x}-a . s .
$$


for every $(s, x) \in F$. Since by Proposition 3.10 there exist measures $\mu, v \in S_{0}$ such that $d K=d \mu(\cdot, X),. d A=d v(\cdot, X$.), the proof is complete.

\subsection{Variational Inequalities}

In this subsection we give some comments on solutions of the obstacle problem in the sense defined in Sections 5.2, 5.4 and solutions in the sense of variational inequalities.

Definition We say that $u \in \mathcal{F}$ is a weak solution of the variational inequality (or solution of the obstacle problem in the variational sense) with terminal condition $\varphi \in H$, generator $f \in \mathcal{H}$ and barriers $h_{1}, h_{2} \in \mathcal{H}\left(\mathrm{VI}\left(\varphi, f, h_{1}, h_{2}\right)\right.$ for short $)$ if

$$
\left\langle v-u, \frac{\partial v}{\partial t}\right\rangle_{T}+\left\langle L_{t} u, v-u\right\rangle_{T}+(f, v-u)_{T} \leq \frac{1}{2}\|\varphi-v(T)\|_{H}^{2}
$$

for every $v \in \mathcal{W}_{\varrho}$ such that $h_{1} \leq v \leq h_{2}$ a.e..

Remark 5.14 In the case of variational inequalities a natural assumption on barriers says that there is $v \in W_{2, e}^{1,1}$ such that $h_{1} \leq v \leq h_{2}$ (see [5]). In general, this assumption leads to solutions different than solutions of the obstacle problem considered in our paper. For instance, if $h_{1}=h_{2} \in W_{2, \varrho}^{1,1}$ then of course $h_{1}$ is a unique weak solution of the variational inequality with barriers $h_{1}, h_{2}$ but in general, $h_{1}$ is not difference of some potentials, so $(\mathrm{H} 4 *)$ is not satisfied. Notice, however, that $(\mathrm{H} 4 *)$ is close to be optimal if we require that solutions of the obstacle problem lead to RBSDEs of the form (1.10) with $\nabla_{\mathbb{X}} u$ replaced by $\nabla u$, because it is shown in [10] that if $u(\cdot, X$.) is a semimartingale then locally $u$ is difference of some potentials.

Remark 5.15 Let us point out that under the assumptions of Theorem 5.13 a unique solution of $\operatorname{OP}\left(\varphi, f, h_{1}, h_{2}\right)$ is a weak solution of $\operatorname{VI}\left(\varphi, f, h_{1}, h_{2}\right)$ if there exists $v \in \mathcal{W}_{\varrho}$ such that $v_{1} \leq v \leq h_{2}$ a.e.. Indeed, taking $v-u_{n}$ as a test function in Eq. 5.12 we easily show that

$$
\left\langle v-u_{n}, \frac{\partial v}{\partial t}\right\rangle_{T}+\left\langle L_{t} u_{n}, v-u_{n}\right\rangle_{T}+\left(f_{u_{n}}, v-u_{n}\right)_{T} \leq \frac{1}{2}\|v(T)-\varphi\|_{H}^{2} .
$$

Using properties of $\left\{u_{n}\right\}$ proved in Theorem 5.13 and taking limes inferior in the above inequality shows that $u$ is a weak solution in the variational sense of the obstacle problem with barriers $h_{1}, h_{2}$.

Remark 5.16 If $f$ does not depend on $z$ and there exists $v \in \mathcal{W}_{\varrho}$ such that $h_{1} \leq$ $v \leq h_{2}$ a.e. then under assumptions (H1)-(H3) there exists a weak solution of $\mathrm{VI}\left(\varphi, f, h_{1}, h_{2}\right)$. Indeed, taking $v-u_{n}$ as a test function in Eq. 5.12 we check that

$$
\sup _{t \in[0, T]}\left\|u_{n}(t)\right\|_{H}^{2}+\left\|\nabla u_{n}\right\|_{\mathcal{H}}^{2} \leq C\left(\|\varphi\|_{H}^{2}+\|g\|_{\mathcal{H}}^{2}+\|v\|_{\mathcal{W}_{e}}^{2}\right)
$$


Hence there exists $u \in \mathcal{F}$ such that $u_{n} \rightarrow u, \nabla u_{n} \rightarrow \nabla u$ weakly in $\mathcal{H}$. Taking once again $v-u_{n}$ as a test function in Eq. 5.12 and using Eq. 5.16 and the following simple estimates

$$
\begin{gathered}
\left(\left(u_{n}-h_{2}\right)^{+}, u_{n}-v\right)_{T} \geq\left\|\left(u_{n}-h_{2}\right)^{+}\right\|_{\mathcal{H}}^{2}, \\
\left(\left(u_{n}-h_{1}\right)^{-}, u_{n}-v\right)_{T} \geq\left\|\left(u_{n}-h_{1}\right)^{-}\right\|_{\mathcal{H}}^{2}
\end{gathered}
$$

we get

$$
\left\|\left(u_{n}-h_{1}\right)^{-}\right\|_{\mathcal{H}}^{2}+\left\|\left(u_{n}-h_{2}\right)^{+}\right\|_{\mathcal{H}}^{2} \leq \frac{C}{n} .
$$

Since $\mathcal{H} \ni u \rightarrow\left\|\left(u-h_{1}\right)^{-}\right\|_{\mathcal{H}}^{2}$ and $\mathcal{H} \ni u \rightarrow\left\|\left(u-h_{2}\right)^{+}\right\|_{\mathcal{H}}^{2}$ are sequentially weakly lower semicontinuous, $h_{1} \leq u \leq h_{2}$ a.e.. Finally, since without loss of generality we may assume that $\mathbb{R} \ni y \rightarrow f(y)$ is monotone, $\mathcal{F} \ni v \rightarrow L_{t} v+f(t, x, v) \in \mathcal{F}^{\prime}$ is pseudo-monotone (see, e.g., [22]). Therefore taking limes inferior in Eq. 5.15 shows that $u$ is a weak solution of $\operatorname{VI}\left(\varphi, f, h_{1}, h_{2}\right)$.

Remark 5.17 In the case of one barrier the condition that there is $v \in \mathcal{W}_{\varrho}$ such that $v \geq h$ a.e. is necessary and sufficient for existence of a unique solution of $\operatorname{OP}(\varphi, f, h)$ (see [17]). Necessity follows from the fact that existence of such $v$ is equivalent to existence of $v \in \mathcal{P}$ such that $v \geq h$ a.e. (see [32]). The latter condition is necessary and sufficient for existence of a minimal solution of $\operatorname{VI}(\varphi, f, h)$, and moreover, the minimal solution coincides with a unique solution of $\operatorname{OP}(\varphi, f, h)$ (see [17]).

In the case of two barriers the condition that there is $v \in \mathcal{W}_{\varrho}$ such that $h_{1} \leq v \leq h_{2}$ a.e. does not imply existence of $\varphi \in \mathcal{P}-\mathcal{P}$ such that $h_{1} \leq \varphi \leq h_{2}$ a.e. and vice versa. In the case of one barrier, a weak solution of $\operatorname{VI}(\varphi, f, h)$ is always a potential, but as shown in Remark 5.14, in general, in the case of two barriers, a weak solution of $\mathrm{VI}\left(\varphi, f, h_{1}, h_{2}\right)$ is not difference of potentials.

It is worth mentioning that in contrast to variational formulation, a solution of $\mathrm{OP}\left(\varphi, f, h_{1}, h_{2}\right)$ if exists is always unique. We know from Remark 5.15 that if there is $v \in \mathcal{W}_{\varrho}$ such that $h_{1} \leq v \leq h_{2}$ a.e., then a unique solution of $\operatorname{OP}\left(\varphi, f, h_{1}, h_{2}\right)$ is a weak solution of $\operatorname{VI}\left(\varphi, f, h_{1}, h_{2}\right)$, but in general, the latter problem may have many other solutions (see [24] for relevant examples). To ensure uniqueness of solutions of variational inequalities additional regularity conditions on the data are needed. If the data are less regular, in the case of one barrier one can cope with the problem of nonuniqueness of solutions by introducing the notion of minimal solutions. Unfortunately, in the case of two barriers, it makes no sense to speak about minimal or maximal solutions.

\section{RBSDEs}

In this section we recall and prove some useful results on general (non-Markovian) reflected BSDEs.

In what follows we assume that we are given a filtered complete probability space $\left(\Omega, \mathcal{F},\left\{\mathcal{F}_{t}\right\}_{t \in[s, T]}, P\right)$ satisfying the usual conditions and carrying a standard $d$-dimensional Wiener process $B$ on $[s, T]$ which has the representation property, i.e. $B$ is an $\left(\left\{\mathcal{F}_{t}\right\}, P\right)$-martingale and for every $\left(\left\{\mathcal{F}_{t}\right\}, P\right)$-square-integrable martingale $M$ on 
$[s, T]$ there is a predictable $d$-dimensional process $H$ such that $E \int_{s}^{T}\left|H_{t}\right|^{2} d t<\infty$ and $M_{t}=\int_{s}^{t} H_{\theta} d B_{\theta}, t \in[s, T]$.

$\mathcal{D}^{2}$ (resp. $\mathcal{S}^{2}$ ) is the space of càdlàg (resp. continuous) progressively measurable processes $\eta$ on $[s, T]$ such that $E \sup _{s \leq t \leq T}\left|\eta_{t}\right|^{2}<\infty$.

$\mathcal{M}$ is the space of progressively measurable processes $\eta$ such that $P\left(\int_{s}^{T}\left|\eta_{t}\right|^{2} d t<\right.$ $\infty)=1, \mathcal{M}^{2}$ is the subspace of $\mathcal{M}$ consisting of processes such that $E \int_{s}^{T}\left|\eta_{t}\right|^{2} d t<\infty$.

$\mathcal{A}^{2}$ is the space of progressively measurable càdlàg increasing processes $K$ such that $E K_{T}^{2}<\infty, \mathcal{A}_{c}^{2}$ is the subspace of $\mathcal{A}^{2}$ consisting of all continuous processes.

Let us consider following hypotheses.

(A1) $\xi \in \mathbb{L}_{2}\left(\Omega, \mathcal{F}_{T}, P\right)$,

(A2) For every $(y, z) \in \mathbb{R} \times \mathbb{R}^{d}$ the process $F(\cdot, y, z)$ is progressively measurable,

(A3) There exist $K>0$ and a process $\gamma \in \mathcal{M}^{2}$ such that

$$
|F(t, y, z)| \leq K\left(\left|\gamma_{t}\right|+|y|+|z|\right), \quad P \text {-a.s. }
$$

for all $t \in[s, T], y \in \mathbb{R}, z \in \mathbb{R}^{d}$

(A4) There is $L>0$ such that

$$
\left|F\left(t, y_{1}, z_{1}\right)-F\left(t, y_{2}, z_{2}\right)\right| \leq L\left(\left|y_{1}-y_{2}\right|+\left|z_{1}-z_{2}\right|\right), \quad P \text {-a.s. }
$$

for every $y_{1}, y_{2} \in \mathbb{R}, z_{1}, z_{2} \in \mathbb{R}^{d}, t \in[s, T]$,

(A5) $S$ is a progressively measurable process such that $E$ ess $\sup _{s \leq t \leq T}\left|S_{t}^{+}\right|^{2}<\infty$.

$\left(\mathrm{A} 5^{*}\right) S$ is a progressively measurable process such that $E$ ess $\sup _{s \leq t \leq T}\left|S_{t}^{-}\right|^{2}<\infty$.

Definition A pair $(Y, Z)$ of $\left\{\mathcal{F}_{t}\right\}$-progressively measurable processes on $[s, T]$ is called a solution of $\operatorname{BSDE}(\xi, F)$ if

(a) $(Y, Z) \in \mathcal{S}^{2} \otimes \mathcal{M}^{2}$,

(b) $Y_{t}=\xi+\int_{t}^{T} F\left(\theta, Y_{\theta}, Z_{\theta}\right)-\int_{t}^{T} Z_{\theta} d B_{\theta}, t \in[s, T], P$-a.s..

Definition A triple $(Y, Z, K)$ of $\left\{\mathcal{F}_{t}\right\}$-progressively measurable processes on $[s, T]$ is a solution of $\underline{\operatorname{RBSDE}}(\xi, F, S)(\operatorname{resp} \cdot \overline{\operatorname{RBSDE}}(\xi, F, S))$ if

(a) $(Y, Z) \in \mathcal{S}^{2} \otimes \mathcal{M}^{2}$,

(b) $Y_{t}=\xi+\int_{t}^{T} F\left(\theta, Y_{\theta}, Z_{\theta}\right)+\int_{t}^{T} d K_{\theta}-\int_{t}^{T} Z_{\theta} d B_{\theta}, t \in[s, T], P$-a.s.,

(c) $Y \geq S$ (resp. $Y \leq S$ ), $d t \otimes P$-a.e.,

(d) $K \in \mathcal{A}^{2}$ (resp. $\left.-K \in \mathcal{A}^{2}\right), K_{0}=0, \int_{s}^{T}\left(Y_{t-}-H_{t-}\right) d K_{t}=0, P$-a.s. for every càdlàg process $H$ such that $S \leq H \leq Y$ (resp. $S \geq H \geq Y$ ), dt $\otimes P$-a.e..

It is known (see [30]) that under (A1)-(A4) there is a unique solution of $\operatorname{BSDE}(\xi, F)$. If, in addition, (A5) (resp. (A $\left.5^{*}\right)$ is satisfied, then there exists a unique solution of $\underline{\operatorname{RBSDE}}(\xi, F, S)$ (resp. $\overline{\operatorname{RBSDE}}(\xi, F, S))$.

In the case of two barriers we consider the following hypothesis.

$L, U \in \mathcal{S}^{2}, L_{t}<U_{t}, t \in[s, T], P$-a.s., $L_{T} \leq \xi \leq U_{T}, P$-a.s..

Definition We say that a triple $(Y, Z, K) \in \mathcal{S}^{2} \otimes \mathcal{M} \otimes\left(\mathcal{A}_{c}-\mathcal{A}_{c}\right)$ is a solution of $\operatorname{RBSDE}(\xi, F, L, U)$ with barriers satisfying (A6) if

(a) $Y_{t}=\xi+\int_{t}^{T} F\left(s, Y_{s}, Z_{s}\right) d t+\int_{t}^{T} d K_{t}-\int_{t}^{T} Z_{s} d B_{s}, t \in[s, T], P$-a.s., 
(b) $L_{t} \leq Y_{t} \leq U_{t}, t \in[s, T], P$-a.s.,

(c) $\int_{s}^{T}\left(U_{t}-Y_{t}\right) d K_{t}^{-}=\int_{s}^{T}\left(Y_{t}-L_{t}\right) d K_{t}^{+}=0, P$-a.s..

It is known (see [15, Theorem 3.7]) that under (A1)-(A4), (A6) there exists a unique solution of $\operatorname{RBSDE}(\xi, F, L, U)$.

Proposition 6.1 Let $(Y, Z, K)$ be a solution of $\operatorname{RBSDE}(\xi, F, L, U)$ with

$$
L_{t}=L_{s}+V_{t}^{1}+\int_{s}^{t} Z_{\theta}^{1} d \theta, \quad U_{t}=U_{s}+V_{t}^{2}+\int_{s}^{t} Z_{\theta}^{2} d \theta, \quad t \in[s, T],
$$

where $V^{i}$ is a finite variation process such that $\left|V^{i}\right| \in \mathcal{A}_{c}^{2}$ and $Z^{i} \in \mathcal{M}^{2}, i=1,2$. Then

$$
\begin{array}{ll}
d K_{t}^{+}+\frac{1}{2} d L_{t}^{0}=\mathbf{1}_{\left\{Y_{t}=L_{t}\right\}}\left(F\left(t, L_{t}, Z_{t}^{1}\right) d t+d V_{t}^{1}\right)^{-}, & \mathbf{1}_{\left\{Y_{t}=L_{t}\right\}}\left(Z_{t}-Z_{t}^{1}\right)=0, \\
d K_{t}^{-}+\frac{1}{2} d L_{t}^{0}=\mathbf{1}_{\left\{Y_{t}=U_{t}\right\}}\left(F\left(t, U_{t}, Z_{t}^{2}\right) d t+d V_{t}^{2}\right)^{+}, & \mathbf{1}_{\left\{Y_{t}=U_{t}\right\}}\left(Z_{t}-Z_{t}^{2}\right)=0,
\end{array}
$$

where $L_{t}^{0}$ is the local time of $Y-L$ and the superscripts +, - mean that in Eqs. 6.1, 6.2 we take positive or negative parts of the Jordan decomposition of signed measures appearing in these formulas.

Proof The proof is similar to that of [12, Proposition 4.2]. By the Itô-Tanaka formula,

$$
\begin{aligned}
d\left(Y_{t}-L_{t}\right)^{+}= & \mathbf{1}_{\left\{Y_{t}>L_{t}\right\}} d\left(Y_{t}-L_{t}\right)+\frac{1}{2} d L_{t} \\
= & -\mathbf{1}_{\left\{Y_{t}>L_{t}\right\}}\left(F\left(t, X_{t}, Z_{t}\right) d t+d V_{t}^{1}\right)+\mathbf{1}_{\left\{Y_{t}>L_{t}\right\}}\left(Z_{t}-Z_{t}^{1}\right) d B_{t} \\
& +\mathbf{1}_{\left\{Y_{t}>L_{t}\right\}} d K_{t}^{-}+\frac{1}{2} d L_{t}^{0} .
\end{aligned}
$$

Since $\left(Y_{t}-L_{t}\right)^{+}=Y_{t}-L_{t}$ and $\mathbf{1}_{\left\{Y_{t}>L_{t}\right\}} d K_{t}^{-}=d K_{t}^{-}$, it follows that

$$
-\mathbf{1}_{\left\{Y_{t}=L_{t}\right\}}\left(F\left(t, Y_{t}, Z_{t}\right) d t+d V_{t}^{1}\right)-d K_{t}^{+}+\mathbf{1}_{\left\{Y_{t}=L_{t}\right\}}\left(Z_{t}-Z_{t}^{1}\right) d B_{t}-\frac{1}{2} d L_{t}^{0}=0
$$

and

$$
-\mathbf{1}_{\left\{Y_{t}=L_{t}\right\}}\left(F\left(t, Y_{t}, Z_{t}\right)+V_{t}^{1}\right) d t-d K_{t}^{+}+\mathbf{1}_{\left\{Y_{t}=L_{t}\right\}}\left(Z_{t}-Z_{t}^{1}\right) d B_{t}-\frac{1}{2} d L_{t}^{0}=0,
$$

which shows Eq. 6.1. In much the same manner we show Eq. 6.2.

As a corollary to Proposition 6.1 we get existence of nonnegative progressively measurable processes $\alpha^{1}, \alpha^{2}$ such that $0 \leq \alpha^{1} \leq 1,0 \leq \alpha^{2} \leq 1$ and

$$
\begin{aligned}
& d K_{t}^{+}=\alpha_{t}^{1} \mathbf{1}_{\left\{Y_{t}=L_{t}\right\}}\left(F\left(t, L_{t}, Z_{t}^{1}\right) d t+d V_{t}^{1}\right)^{-}, \\
& d K_{t}^{-}=\alpha_{t}^{2} \mathbf{1}_{\left\{Y_{t}=U_{t}\right\}}\left(F\left(t, U_{t}, Z_{t}^{2}\right) d t+d V_{t}^{2}\right)^{+} .
\end{aligned}
$$

Let us consider yet another hypothesis.

(A6*) There exists a semimartingale $H \in \mathcal{D}^{2}$ such that $H_{t}=H_{s}+\int_{s}^{t} d K_{\theta}^{0}-$ $\int_{s}^{t} d A_{\theta}^{0}+\int_{s}^{t} Z^{0} d B_{\theta}$ for some $A^{0}, K^{0} \in \mathcal{A}^{2}, Z^{0} \in \mathcal{M}^{2}$, and $L_{t} \leq H_{t} \leq U_{t}$ for a.e. $t \in[s, T], P$-a.s.. 
Definition We say that a triple $(Y, Z, K) \in \mathcal{D}^{2} \otimes \mathcal{M}^{2} \otimes\left(\mathcal{A}^{2}-\mathcal{A}^{2}\right)$ is a solution of $\operatorname{RBSDE}(\xi, F, L, U)$ with barriers satisfying (A6*) if

(a) $Y_{t}=\xi+\int_{s}^{T} F\left(\theta, Y_{\theta}, Z_{\theta}\right) d \theta+\int_{s}^{T} d K_{\theta}-\int_{s}^{T} Z_{\theta} d B_{\theta}, t \in[s, T], P$-a.s.

(b) $L_{t} \leq Y_{t} \leq U_{t}$ for a.e. $t \in[s, T], P$-a.s.,

(c) $\int_{s}^{T}\left(U_{t-}^{*}-Y_{t-}\right) d K_{t}^{-}=\int_{s}^{T}\left(Y_{t-}-L_{t-}^{*}\right) d K_{t}^{+}=0, P$-a.s. for every $L^{*}, U^{*} \in \mathcal{D}^{2}$ such that $L_{t} \leq L_{t}^{*} \leq Y_{t} \leq U_{t}^{*} \leq U_{t}$ for a.e. $t \in[s, T], P$-a.s..

It is known (see [30, Theorem 2.3]) that under (A1)-(A4), (A6*) there exists a unique solution of $\operatorname{RBSDE}(\xi, F, L, U)$.

Remark 6.2 It is worth mentioning that if the barriers $L, U$ satisfy (A6) and (A6*), then the definition preceding Proposition 6.1 is equivalent to the above definition of a solution $\operatorname{RBSDE}(\xi, F, L, U)$.

Given a sequence $\left\{x_{n}\right\}$ of elements of some vector space we write

$$
\sigma_{m}\left(\left\{x_{n}\right\}\right)=\frac{1}{m} \sum_{i=1}^{m} x_{i}, \quad m \in \mathbb{N} .
$$

Theorem 6.3 Assume that (A1)-(A4), (A6*) hold. Then there exists a unique solution $(Y, Z, K)$ of $\operatorname{RBSDE}(\xi, F, L, U)$. Moreover, if the pair $\left(Y^{n, m}, Z^{n, m}\right)$ is a unique solution of $B \operatorname{SDE}\left(\xi, F_{n, m}\right)$ with $F_{n, m}(t, y, z)=F(t, y, z)+n\left(y-L_{t}\right)^{-}-m\left(y-U_{t}\right)^{+}$, then

(i) There exists $C>0$ such that

$$
\begin{gathered}
E \sup _{s \leq t \leq T}\left|Y_{t}^{n, m}\right|^{2}+\int_{s}^{T}\left|Z_{t}^{n, m}\right|^{2} d t+E\left|K_{T}^{n, m}\right|^{2}+E\left|A_{T}^{n, m}\right|^{2} \\
\leq C\left(E|\xi|^{2}+E\left|K_{T}^{0}\right|^{2}+E\left|A_{T}^{0}\right|^{2}+E \int_{s}^{T}\left|\gamma_{t}\right|^{2} d t\right. \\
\quad+\underset{s \leq t \leq T}{\left.E \operatorname{ess} \sup _{t}\left|L_{t}^{+}\right|^{2}+E \underset{s \leq t \leq T}{\operatorname{ess} \sup _{t}}\left|U_{t}^{-}\right|^{2}\right)}
\end{gathered}
$$

where

$$
\begin{aligned}
& K_{t}^{n, m}=\int_{s}^{t} n\left(Y_{\theta}^{n, m}-L_{\theta}\right)^{-} d \theta, \quad t \in[s, T], \quad P \text {-a.s., } \\
& A_{t}^{n, m}=\int_{s}^{t} m\left(Y_{\theta}^{n, m}-U_{\theta}\right)^{+} d \theta, \quad t \in[s, T], \quad P \text {-a.s. },
\end{aligned}
$$

(ii) $Y_{t}^{n, n} \rightarrow Y_{t} t \in[s, T]$, P-a.s. and $Y^{n, n} \rightarrow Y$ in $\mathcal{M}^{2}, Z^{n, n} \rightarrow Z$ in $\mathcal{M}^{p}$ for $p \in$ $[1,2)$,

(iii) There exists a unique solution $\left(\bar{Y}^{n}, \bar{Z}^{n}, \bar{A}^{n}\right)$ of $\bar{R} B S D E\left(\xi, \bar{F}_{n}, U\right)$ with $\bar{F}_{n}(t, y, z)=F(t, y, z)+n\left(y-L_{t}\right)^{-}$and

$$
d \bar{A}^{n} \rightarrow d K^{-} \quad \text { in probability } P,
$$


$\bar{Y}_{t}^{n} \nearrow Y_{t}, t \in[s, T], P$-a.s., $\bar{Y}^{n} \rightarrow Y$ in $\mathcal{M}^{2}, \bar{Z}^{n} \rightarrow Z$ in $\mathcal{M}^{p}$ for $p \in[1,2)$, and for some subsequence $\left\{n_{k}\right\}$,

$$
\sigma_{k}\left(\left\{d \bar{K}^{n_{k}}\right\}\right) \rightarrow d K^{+} \quad \text { in probability } P,
$$

where $\bar{K}_{t}^{n}=\int_{s}^{t} n\left(\bar{Y}_{\theta}^{n}-L_{\theta}\right)^{-} d \theta$. Moreover, Eq. 6.3 is satisfied with $\left(\bar{Y}^{n}, \bar{Z}^{n}\right.$, $\left.\bar{K}^{n}, \bar{A}^{n}\right)$ in place of $\left(Y^{n, m}, Z^{n, m}, K^{n, m}, A^{n, m}\right)$. Analogous assertions hold for the quadruple $\left(\underline{Y}^{n}, \underline{Z}^{n}, \underline{K}^{n}, \underline{A}^{n}\right)$, where $\left(\underline{Y}^{n}, \underline{Z}^{n}, \underline{K}^{n}\right)$ is a unique solution of $\underline{R B S D E}\left(\xi, \underline{F}_{n}, U\right)$ with $\underline{F}_{n}(t, y, z)=F(t, y, z)-n\left(y-U_{t}\right)^{+}$.

(iv) If the obstacles $L, U$ are continuous then $\bar{Z}_{n} \rightarrow Z, Z^{n} \rightarrow Z$ in $\mathcal{M}^{2}$ and $d \bar{K}^{n} \rightarrow$ $d K^{+}, d \bar{A}^{n} \rightarrow d K^{-}, d \underline{K}^{n} \rightarrow d K^{+}, d \underline{A}^{n} \rightarrow d K^{-}$in probability $P$.

Proof A brief inspection of the proof of [30, Proposition 5.1] reveals that Eq. 6.3 is satisfied.

(ii) Convergence of $\left\{Y^{n, n}\right\}$ follows from [30, Theorem 6.2]. To prove convergence of $\left\{Z^{n, n}\right\}$ let us first observe that by Itô's formula, for any stopping times $\sigma, \tau$ such that $s \leq \sigma \leq \tau \leq T$ we have

$$
\begin{aligned}
E \mid Y_{\tau}^{n, n}- & \left.Y_{\tau}\right|^{2}+E \int_{\sigma}^{\tau}\left|Z_{t}^{n, n}-Z_{t}\right|^{2} d t+E \sum_{\sigma<t \leq \tau}\left|\Delta Y_{t}\right|^{2} \\
= & E\left|Y_{\sigma}^{n, n}-Y_{\sigma}\right|^{2}+2 E \int_{\sigma}^{\tau}\left(F\left(t, Y_{t}^{n, n}, Z_{t}^{n, n}\right)-F\left(t, Y_{t}, Z_{t}\right)\right)\left(Y_{t}^{n, n}-Y_{t}\right) d t \\
& +2 E \int_{\sigma}^{\tau}\left(Y_{t}^{n, n}-Y_{t-}\right) d\left(K_{t}^{n, n}-K_{t}\right)-2 E \int_{\sigma}^{\tau}\left(Y_{t}^{n, n}-Y_{t-}\right) d\left(A_{t}^{n, n}-A_{t}\right) \\
= & E\left|Y_{\sigma}^{n, n}-Y_{\sigma}\right|^{2}+2 E \int_{\sigma}^{\tau}\left(F\left(t, Y_{t}^{n, n}, Z_{t}^{n, n}\right)-F\left(t, Y_{t}, Z_{t}\right)\right)\left(Y_{t}^{n, n}-Y_{t}\right) d t \\
& +2 E \int_{\sigma}^{\tau}\left(Y_{t}^{n, n}-Y_{t}\right) d\left(K_{t}^{n, n}-K_{t}\right)-2 E \int_{\sigma}^{\tau}\left(Y_{t}^{n, n}-Y_{t}\right) d\left(A_{t}^{n, n}-A_{t}\right) \\
& +2 E \sum_{\sigma<t \leq \tau}\left|\Delta Y_{t}\right|^{2},
\end{aligned}
$$

the last inequality being a consequence of the fact that $\Delta Y_{t}^{n, n}=\Delta A_{t}^{n, n}=$ $\Delta K_{t}^{n, n}=0$ and $\Delta Y_{t}=\Delta A_{t}-\Delta K_{t}$. By elementarny calculations we also have

$$
\int_{\sigma}^{\tau}\left(Y_{t}^{n, n}-Y_{t}\right) d\left(K^{n, n}-K_{t}\right) \leq-\int_{\sigma}^{\tau}\left(Y_{t}^{n, n}-Y_{t}\right) d K_{t}
$$

and

$$
\int_{\sigma}^{\tau}\left(Y_{t}^{n, n}-Y_{t}\right) d\left(A^{n, n}-A_{t}\right) \leq \int_{\sigma}^{\tau}\left(Y_{t}^{n, n}-Y_{t}\right) d A_{t} .
$$


By the above,

$$
\begin{aligned}
E \int_{\sigma}^{\tau} \mid & Z_{t}^{n, n}-\left.Z_{t}\right|^{2} d t \\
\quad \leq & E\left|Y_{\tau}^{n, n}-Y_{\tau}\right|^{2}+2 E \int_{\sigma}^{\tau}\left|F\left(t, Y_{t}^{n, n}, Z_{t}^{n, n}\right)-F\left(t, Y_{t}, Z_{t}\right)\right|\left|Y_{t}^{n, n}-Y_{t}\right| d t \\
& \quad+E \int_{\sigma}^{\tau}\left|Y_{t}^{n, n}-Y_{t}\right| d\left(K_{t}+A_{t}\right)+2 E \sum_{\sigma<t \leq \tau}\left|\Delta A_{t}\right|^{2}+2 E \sum_{\sigma<t \leq \tau}\left|\Delta K_{t}\right|^{2} .
\end{aligned}
$$

To show convergence of $\left\{Z^{n, n}\right\}$ in $\mathcal{M}^{p}$ for $p \in[1,2)$ it suffices now to repeat arguments following Eq. 2.7 in the proof of [29, Theorem 2.1].

(iii) We show (iii) only in the case of upper barrier. Existence of a solution $\left(\bar{Y}^{n}, \bar{Z}^{n}, \bar{A}^{n}\right)$ of $\overline{\operatorname{RBSDE}}\left(\xi, \bar{F}_{n}, U\right)$ follows from [30, Theorem 2.1]. From the same theorem it follows that for each $n \in \mathbb{N}$, if $m \rightarrow \infty$ then $\left\{\left(Y^{n, m}, Z^{n, m}, K^{n, m}, A^{n, m}\right)\right\}$ converges to the process $\left(\bar{Y}^{n}, \bar{Z}^{n}, \bar{K}^{n}, \bar{A}^{n}\right)$ in such spaces that letting $m \rightarrow \infty$ in Eq. 6.3 implies that Eq. 6.3 is satisfied with $\left\{\left(Y^{n, m}, Z^{n, m}, K^{n, m}, A^{n, m}\right)\right\}$ replaced by $\left(\bar{Y}^{n}, \bar{Z}^{n}, \bar{K}^{n}, \bar{A}^{n}\right)$. Convergence of $\left\{\bar{Y}^{n}\right\}$, $\left\{\bar{Z}^{n}\right\}$ follows from (65), (66) in [30]. Next, from (61) in [30] (see also [29, Lemma 2.2]) we conclude that there exists $\tilde{A} \in \mathcal{A}^{2}$ such that $\bar{A}_{t}^{n} \rightarrow \tilde{A}_{t}, t \in[s, T], P$ a.s.. From this it follows in particular that $d \bar{A}^{n} \rightarrow d \tilde{A}, P$-a.s.. Since $\left\{\bar{Z}^{n}\right\}$ is weakly convergent to $Z$ in $\mathbb{L}_{2}(\Omega \times[s, T], P \otimes \lambda)$ and $\mathbb{L}_{2}(\Omega \times[s, T], P \otimes \lambda)$ as the Hilbert space has the Banach-Saks property, there exists a subsequence $\left\{\bar{Z}^{n_{k}}\right\}$ such that $\sigma_{k}\left(\left\{\bar{Z}^{n_{k}}\right\}\right) \rightarrow Z$ strongly in $\mathbb{L}_{2}(\Omega \times[s, T], P \otimes \lambda)$ as $k \rightarrow+\infty$. Using this and the Burkholder-Davis-Gundy inequality we conclude that

$$
\int_{s} \sigma_{k}\left(\left\{\bar{Z}_{\theta}^{n_{k}}\right\}\right) d B_{\theta} \rightarrow \int_{s}^{\cdot} Z_{\theta} d B_{\theta}
$$

in $\mathcal{S}^{2}$. Furthermore, from convergence of $\left\{\bar{Y}^{n}\right\},\left\{\bar{Z}^{n}\right\},\left\{\bar{A}^{n}\right\}$ it follows that $P$-a.s.,

$$
\int_{s}^{t} \sigma_{k}\left(\left\{F\left(\theta, \bar{Y}_{\theta}^{n_{k}}, \bar{Z}_{\theta}^{n_{k}}\right)\right\}\right) d \theta \rightarrow \int_{s}^{t} F\left(\theta, Y_{\theta}, Z_{\theta}\right) d \theta \text { uniformly in } t \in[s, T]
$$

and

$$
\sigma_{k}\left(\left\{\bar{Y}_{t}^{n_{k}}\right\}\right) \rightarrow Y_{t}, \quad \sigma_{k}\left(\left\{\bar{A}_{t}^{n_{k}}\right\}\right) \rightarrow \tilde{A}_{t}, t \in[s, T], \text { P-a.s. }
$$

as $k \rightarrow+\infty$. From Eqs. 6.5-6.7 we conclude that there exists $\tilde{K} \in \mathcal{A}^{2}$ such that $\sigma_{k}\left(\left\{\bar{K}_{t}^{n_{k}}\right\}\right) \rightarrow \tilde{K}_{t}, t \in[s, T], P$-a.s. as $k \rightarrow+\infty$, which implies that $\sigma_{k}\left(\left\{d \bar{K}^{n_{k}}\right\}\right) \rightarrow$ $d \tilde{K}$. Write $K=\tilde{K}-\tilde{A}$. We are going to show that $\tilde{K}=K^{+}$and $\tilde{A}=K^{-}$. We know that

$$
Y_{t}=\xi+\int_{t}^{T} F\left(\theta, Y_{\theta}, Z_{\theta}\right) d \theta+\int_{t}^{T} d K_{\theta}^{+}-\int_{t}^{T} d K_{\theta}^{-}-\int_{t}^{T} Z_{\theta} d B_{\theta}, \quad t \in[s, T] .
$$

Let $\left(S^{m}, V^{m}\right)$ be a solution of the BSDE

$$
S_{t}^{m}=\xi+\int_{t}^{T} F\left(\theta, S_{\theta}^{m}, V_{\theta}^{m}\right) d \theta+\int_{t}^{T} d K_{\theta}^{+}-\int_{t}^{T} d \tilde{A}_{\theta}^{m}-\int_{t}^{T} V_{\theta}^{m} d B_{\theta},
$$


where $d \tilde{A}_{t}^{m}=m\left(S_{t}^{m}-U_{t}\right)^{+} d t$. By the comparison theorem for BSDEs (since the term $-\int_{t}^{T} m\left(Y_{\theta}-U_{\theta}\right)^{+} d \theta$ is equal to zero we may add it to the right-hand side of Eq. 6.8), $Y_{t} \leq S_{t}^{m}, t \in[s, T], P$-a.s.. Hence $L \leq S^{m}$. Therefore adding the term $\int_{t}^{T} n\left(S_{\theta}^{n}-L_{\theta}\right)^{-} d \theta=0$ to Eq. 6.9 and using once again the comparison theorem for BSDEs we get

$$
Y_{t}^{n, m} \leq S_{t}^{m}, \quad t \in[s, T], \quad P \text {-a.s. },
$$

which implies that

$$
d A^{n, m} \leq d \tilde{A}^{m}, \quad P-a . s . .
$$

Since the pair $\left(S^{m}, V^{m}\right)$ is a unique solution of the penalization problem for the equation $\overline{\operatorname{RBSDE}}\left(\xi, F d t+d K_{t}^{+}, U\right)$, it follows from results proved in [30] that $\left\{\left(S^{m}, V^{m}, \tilde{A}^{m}\right)\right\}$ converges to $(S, V, \bar{A})$ in the sense of (ii), where $(S, V, \bar{A})$ is a solution of $\overline{\operatorname{RBSDE}}\left(\xi, f d t+d K_{t}^{+}, U\right)$. Hence $S \leq U$ and $\bar{A}$ satisfies the minimality condition (c) in the definition following (A6*). Moreover, from Eq. 6.10 it follows that $L \leq S$ and since $d K^{+} \leq d \tilde{K}, K^{+}$satisfies the minimality condition. Therefore $\left(S, V, K^{+}-\bar{A}\right)$ is a solution of $\operatorname{RBSDE}(\xi, F, L, U)$. By uniqueness, $K^{+}-\bar{A}=K$, and hence $\bar{A}=K^{-}$. By [17], without loss of generality we may assume that $\sigma_{m}\left(\left\{d A^{n, m}\right\}\right) \rightarrow d \bar{A}^{n}, \sigma_{m}\left(\left\{d \tilde{A}^{m}\right\}\right) \rightarrow d \bar{A}$ in probability $P$. From this and Eq. 6.11 it follows that $d \bar{A}^{n} \leq d \bar{A}$, which when combined with Eq. 6.7 yields $d \tilde{A} \leq d \bar{A}=d K^{-}$. Therefore $\tilde{A}=K^{-}$by the minimality property of the Jordan decomposition. In the same manner one can prove that $\tilde{K}=K^{-}$.

(iv) If the barriers are continuous then $Y$ and $K, A$ are continuous (see, e.g., [45, Lemma 2.1]). Hence $\Delta K_{t}=\Delta A_{t}=0$ for $t \in[s, T]$, which when combined with Eq. 6.4 and convergence of $\left\{Y^{n}\right\}$ shows convergence of $\left\{Z^{n}\right\}$ to $Z$ in $\mathcal{M}^{2}$. Convergence of $\left\{\bar{Z}_{n}\right\}$ follows from [30, Theorem 3.1] and continuity of the process $K$.

Open Access This article is distributed under the terms of the Creative Commons Attribution License which permits any use, distribution, and reproduction in any medium, provided the original author(s) and the source are credited.

\section{References}

1. Andreianov, B., Sbihi, K., Wittbold, P.: On uniqueness and existence of entropy solutions for nonlinear parabolic problems with absorption. J. Evol. Equ. 8, 449-490 (2008)

2. Aronson, D.G.: Non-negative solutions of linear parabolic equations. Ann. Sc. Norm. Super. Pisa 22, 607-693 (1968)

3. Bally, V., Matoussi, A.: Weak solutions for SPDEs and backward doubly stochastic differential equations. J. Theoret. Probab. 14, 125-164 (2001)

4. Bénilan, P., Boccardo, L., Gallouet, T., Gariepy, R., Pierre, M. and Vazquez, J.L.: An L1 theory of existence and uniqueness of solutions of nonlinear elliptic equations. Ann. Sc. Norm. Super. Pisa, Cl. Sci. 22, 240-273 (1995)

5. Bensoussan, A., Lions J.-L.: Applications of Variational Inequalities in Stochastic Control. North-Holland, Amsterdam (1982)

6. Blumenthal, M.R., Getoor, R.K.: Markov Processes and Potential Theory. Dover Publications, New York (2007)

7. Briand, Ph., Delyon, B., Hu, Y., Pardoux, E., Stoica, L.: $L^{p}$ solutions of backward stochastic differential equations. Stoch. Process. Their Appl. 108, 109-129 (2003) 
8. Buckdahn, R., Li, J.: Probabilistic interpretation for systems of Isaacs equations with two reflecting barriers. Nonlinear Differ. Equ. Appl. 16, 381-420 (2009)

9. Chen, Q.: Optimal obstacle control problem for semilinear evolutionary bilateral variational inequalities. J. Math. Anal. Appl. 307, 677-690 (2005)

10. Cinlar, E., Jacod, J., Protter, P., Sharpe, M.J.: Semimartingales and Markov processes. Z. Wahrscheinlichkeitstheor. Verw. Geb. 54, 161-219 (1980)

11. Droniou, J., Poretta, A., Prignet, A.: Parabolic capacity and soft measures for nonlinear equations. Potential Anal. 19, 99-161 (2003)

12. El Karoui, N., Kapoudjian, C., Pardoux, E., Peng, S., Quenez, M.C.: Reflected solutions of backward SDEs, and related obstacle problems for PDE's. Ann. Probab. 25, 702-737 (1997)

13. Fukushima, M., Oshima, Y., Takeda, M.: Dirichlet Forms and Symmetric Markov Processes. De Gruyter Studies in Mathematics 19. Walter de Gruyter, New York (1994)

14. Getoor, R.K., Sharpe, M.J.: Naturality, standardness, and weak duality for Markov processes. Z. Wahrscheinlichkeitstheor. Verw. Geb. 67, 1-62 (1984)

15. Hamadène, S., Hassani, M.: BSDEs with two reflecting barriers: the general result. Probab. Theory Relat. Fields 132, 237-264 (2005)

16. Klimsiak, T.: On time-dependent functionals of diffusions corresponding to divergence form operators. J. Theor. Probab. doi:10.1007/s10959-011-0381-4 (2011)

17. Klimsiak, T.: Reflected BSDEs and the obstacle problem for semilinear PDEs in divergence form. Stoch. Process. Their Appl. 122, 134-169 (2012)

18. Kubo, M.: Variational inequalities with time-dependent constraints in $L^{p}$. Nonlinear Anal. 73, 390-398 (2010)

19. Kubo, M., Yamzaki, N.: Periodic solutions of elliptic-parabolic variational inequalities with timedependent constraints. J. Evol. Equ. 6, 71-93 (2006)

20. Ladyzenskaya, O.A., Solonnikov, V.A., Ural'ceva, N.N.: Linear and quasi-linear equations of parabolic type. Transl. Math. Monographs 23, Am. Math. Soc., Providence, R.I. (1968)

21. Lejay, A.: A probabilistic representation of the solution of some quasi-linear PDE with a divergence form operator. Application to existence of weak solutions of FBSDE. Stoch. Process. Their Appl. 110, 145-176 (2004)

22. Lions, J.-L.: Quelques Méthodes de Résolutions des Problèmes aux Limites Non Linéaires. Dunod, Gauthier Villars, Paris (1969)

23. Lyons, T.J., Zheng, W.A.: On conditional diffusion processes Proc. Roy. Soc, Edinburgh 115, 243-255 (1990)

24. Mignot, F., Puel, J.P.: Inéquations d'évolution paraboliques avec convexes dépendant du temps. Applications aux inéquations quasi-variationnelles d'évolution. Arch. Ration. Mech. Anal. 64, 59-91 (1977)

25. Mokrane, A., Murat, F.: The Lewy-Stampacchia inequality for bilateral problems. Ric. Mat. 53, 139-182 (2004)

26. Oshima, Y.: Time-dependent Dirichlet forms and related stochastic calculus. Infin. Dimens. Anal. Quantum Probab. Relat. Top. 7, 281-316 (2004)

27. Ouknine, Y., Ndiaye, D.: Weak solutions of semilinear PDEs with obstacle(s) in Sobolev spaces and their probabilistic interpretation via the RFBSDEs and DRFBSDEs. Stoch. Dyn. 2, 247-269 (2008)

28. Palmeri, M.C.: Homographic approximation for some nonlinear parabolic unilateral problems. J. Convex Anal. 7, 353-373 (2000)

29. Peng, S.: Monotonic limit theorem of BSDE and nonlinear decomposition theorem of DoobMeyers type. Probab. Theory Relat. Fields 113, 473-499 (1999)

30. Peng, S., Xu, M.: The smallest g-supermartingale and reflected BSDE with single and double L2 obstacles. Ann. I.H. Poincare 41, 605-630 (2005)

31. Pierre, M.: Problemes d'evolution avec contraintes unilatérales et potentiel paraboliques. Comm. Partial Differential Equations 4, 1149-1197 (1979)

32. Pierre, M.: Parabolic capacity and Sobolev spaces. J. Math. Anal. 14, 522-533 (1983)

33. Ren, Y., Xia, N.: Generalized reflected BSDE and an obstacle problem for PDEs with nonlinear neumann boundary condition. Stoch. Anal. Appl. 24, 1013-1033 (2006)

34. Revuz, D.: Mesures associees aux fonctionnelles additives de Markov I. Trans. Am. Math. Soc. 148, 501-531 (1970)

35. Revuz, D., Yor, M.: Continuous Martingales and Brownian Motion. Springer, Berlin (1991)

36. Rozkosz, A.: Weak convergence of diffusions corresponding to divergence form operators. Stoch. Stoch. Rep. 57, 129-157 (1996) 
37. Rozkosz, A.: On Dirichlet processes associated with second order divergence form operators. Potential Anal. 14, 123-149 (2001)

38. Rozkosz, A.: Time-inhomogeneous diffusions corresponding to symmetric divergence form operators. Probab. Math. Stat. 22, 231-252 (2002)

39. Rozkosz, A.: Backward SDEs and Cauchy problem for semilinear equations in divergence form. Probab. Theory Relat. Fields 125, 393-401 (2003)

40. Rozkosz, A.: On the Feynman-Kac representation for solutions of the Cauchy problem for parabolic equations in divergence form. Stochastics 77, 297-313 (2005)

41. Stannat, W.: Dirichlet forms and Markov processes: a generalized framework including both elliptic and parabolic cases. Potential Anal. 8, 2-60 (1998)

42. Stannat, W.: The theory of generalized Dirichlet forms and its application in analysis and stochastics. Mem. Am. Math. Soc. 142, viii+101 (1999)

43. Stoica, I.L.: A Probabilistic interpretation of the divergence and BSDE's. Stoch. Process. Their Appl. 103, 31-55 (2003)

44. Stroock, D.W.: Diffusion semigroups corresponding to uniformly eliptic divergence form operators. In: Seminaire de Probabilities XXII. Lecture Notes in Math., vol. 1321, pp. 316-347 (1988)

45. Shiqiu, Z., Shengwu, Z.: A generalized existence theorem of reflected BSDEs with double obstacles. Stat. Probab. Lett. 78, 528-536 (2008) 